Review

\title{
Structure and Morphology Control in Thin Films of Conjugated Polymers for an Improved Charge Transport
}

\author{
Haiyang Wang ${ }^{1,2}$, Yaozhuo Xu ${ }^{1,2}$, Xinhong Yu ${ }^{1}$, Rubo Xing ${ }^{1}$, Jiangang Liu ${ }^{1}$ and \\ Yanchun Han ${ }^{1, *}$
}

1 State Key Laboratory of Polymer Physics and Chemistry, Changchun Institute of Applied Chemistry, Chinese Academy of Sciences, 5625 Renmin Street, Changchun 130022, China;

E-Mails: hywang@ciac.ac.cn (H.W.); xuyaozhuo@ciac.ac.cn (Y.X.); xhyu@ciac.ac.cn (X.Y.); rbxing@ciac.ac.cn (R.X.); niitawh@ciac.ac.cn (J.L.)

2 University of the Chinese Academy of Sciences, No. 19A Yuquan Road, Beijing 100049, China;

* Author to whom correspondence should be addressed; E-Mail: ychan@ciac.ac.cn;

Tel.: +86-431-8526-2175; Fax: +86-431-8526-2126.

Received: 26 September 2013; in revised form: 24 October 2013 / Accepted: 31 October 2013 / Published: 18 November 2013

\begin{abstract}
The morphological and structural features of the conjugated polymer films play an important role in the charge transport and the final performance of organic optoelectronics devices [such as organic thin-film transistor (OTFT) and organic photovoltaic cell (OPV), etc.] in terms of crystallinity, packing of polymer chains and connection between crystal domains. This review will discuss how the conjugated polymer solidify into, for instance, thin-film structures, and how to control the molecular arrangement of such functional polymer architectures by controlling the polymer chain rigidity, polymer solution aggregation, suitable processing procedures, etc. These basic elements in intrinsic properties and processing strategy described here would be helpful to understand the correlation between morphology and charge transport properties and guide the preparation of efficient functional conjugated polymer films correspondingly.
\end{abstract}

Keywords: conjugated polymer; thin film; morphology; structure; chain rigidity; solution aggregation; processing procedures 


\section{Introduction}

Recently, $\pi$-conjugated polymers have attracted great attention due to their extensive applications in electronic devices such as field-effect transistors (FETs), organic photovoltaic cells (OPVs), organic light-emitting diodes (OLEDs) and so on [1-7]. Compared with inorganic semiconductors and organic $\pi$-conjugated small molecules, the family of conjugated polymers has great advantages in its low weight, easy solution-processing and suitability to industrial manufacturing of large-area devices such as roll-to-roll routes [8-10]. Thus, many groups of conjugated polymers have been synthesized [3,11-16] and a extremely high carrier mobility of $12.04 \mathrm{~cm}^{2} \cdot \mathrm{V}^{-1} \cdot \mathrm{s}^{-1}$ in FETs without doping [17] and power conversion efficiency of $8.67 \%$ in single junction OPVs [18] have been realized among them, which shows a brilliant prospects of them as the next-generation electronic materials. Therefore, understanding the correlations between the intrinsic properties of conjugated polymers, the features of thin film and the performance of corresponding electronic devices become crucial to guide the synthesis of more excellent materials and achievement of better electronic properties.

It is well known that an important origin for electronic properties of conjugated polymers based devices is the delocalization of $\pi$-electrons in and between the backbones, which leads to the aggregation/crystallization of polymer chains and the formation of various morphology further. The gradual bottom-up construction of $\pi$-conjugation system provides the transport pathways of charges. However, clarifying the detailed, bottom-up relationship between materials and performance has been always challenging since the discovery of semi-conducting conjugated polymers. After the related research for several decades, a great progress has been made in this field [2,3,19-29], i.e., proposing more clear and efficient synthesis guidelines, concluding principles to improve morphological order and revealing the importance of morphological and structural features in films to the final performance. Now, synthesis of more efficient $\pi$-conjugated systems and improvement of order in films have become the essential problems for the development of opto-electronic functional conjugated polymers in terms of chemistry and physics, respectively.

In this review, we focus on the importance of controlling morphology and structure in films to the final performance of conjugated polymer films, which is the bridge between materials and properties Firstly, the basic morphological and structural features influencing the charge transport properties will be discussed. Secondly, the intrinsic conjugation properties as the crucial factors to determine the final film morphology and performance will be demonstrated. Thirdly, as solution-processable materials, the solution state of conjugated polymers will be discussed and the universal strategy to improve solution state will be proposed. This review is thought helpful to the design, processing and applications of semiconductor conjugated polymers.

\section{The Morphological and Structural Features Influencing the Charge Transport in Films}

As mentioned above, the origin of electronic properties of conjugated polymer functional films is the stepwise bottom-up construction from $\pi$-conjugated units at molecular scale to continuous pathways for charge transport at meso-, micro- and even macroscopic scale (Figure 1). Firstly, although the electronic conjugation arises from the $\pi-\pi$ interaction between pendant conjugated side groups in some polymers with flexible main-chains such as poly(9-vinylcarbazole), the intra-chain conjugation 
and inter-chain interaction accounting for charge transport originate from the delocalization of $\pi$-electrons in backbones and their overlap between adjacent backbones for most conjugated polymers, respectively. In this work, the attention will be paid into the polymer family with conjugated backbones. Then, the two driving forces make conjugated polymer chains stack into ordered crystalline regions. In the process of crystallization, different molecular structures and processing procedures result in self-assembly of chains along different thermodynamic and/or kinetic pathways and formation of various crystalline morphologies. According to the bottom-up construction of charge transport pathways, (1) the internal molecular order in crystalline regions at molecular scale ( $1 \AA-10 \mathrm{~nm}$ ) (Figure 1a); (2) the connectivity between crystalline regions at meso- and microscopic scale $(10 \mathrm{~nm}-1 \mu \mathrm{m})$ (Figure 1b,c); (3) the amount of crystallites and alignment of crystalline regions at microscopic and device size $(100 \mathrm{~nm}-100 \mu \mathrm{m})$ (Figure 1c) are important primary morphological and structural features to influence the continuous and efficient charge transport between electrodes. They will be discussed in detail in following.

Figure 1. Scheme of the bottom-up construction of charge transport pathways with the morphological features influencing the final morphology and performances in conjugated polymer films: (a) the molecular stacking order; (b) the connectivity between adjacent crystalline regions and (c) the grain boundary angle and alignment of charge transport pathways.
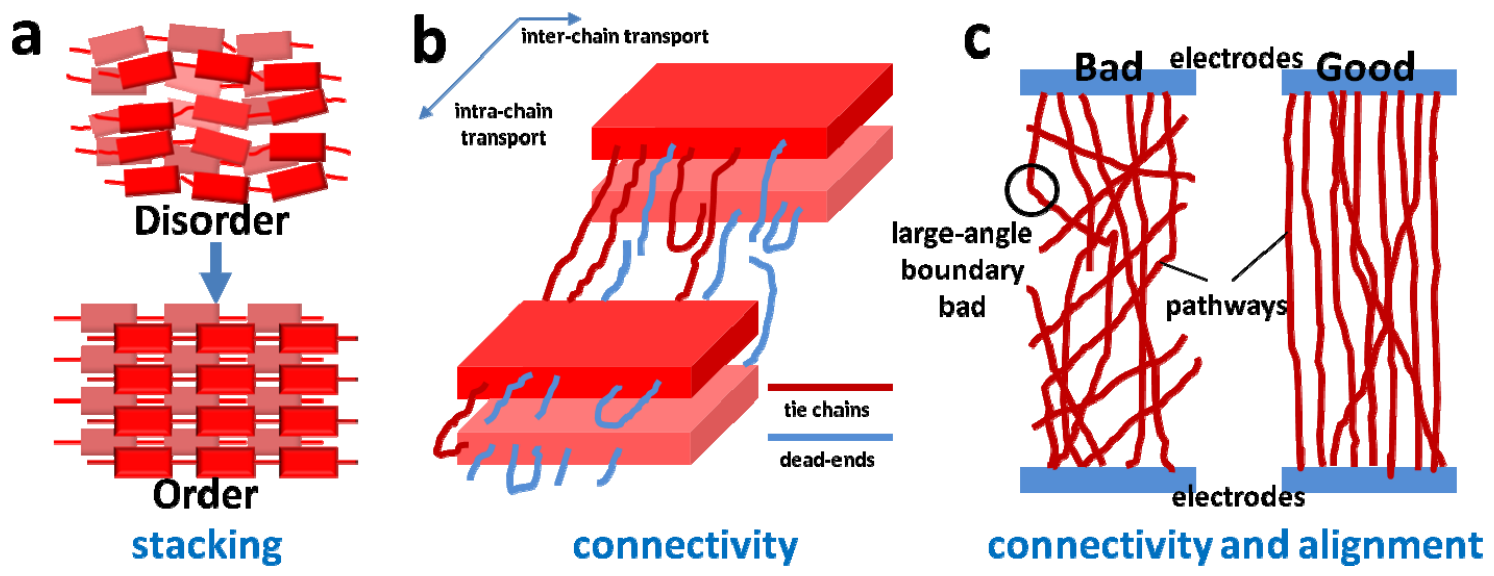

\subsection{The Order of Crystalline Regions}

A good internal order in crystallites of conjugated polymers could behave as extended intra-chain conjugation and ordered inter-chain stacking (Figure 1a), which could be reflected in photophysical features in UV-Vis absorption and photoluminescence spectra, i.e., H-aggregation and J-aggregation [30-32]. In terms of electronic, H-aggregation means that the sign of the resonant electronic coupling is positive, the main absorption peak shifts to higher energies and fluorescence is quenched, while J-aggregation means that the couplings are negative and a spectral red shift happens. Meanwhile, the ratio of $A_{0-0} / A_{0-1}$ in absorption spectra and/or $0-0$ emission/0-1 emission in photoluminescence spectra (Figure 2) could reflect the order in molecular aggregations according to Equation (1) [30,32] as follows,

$$
R_{\mathrm{abs}}=\frac{A_{0-0}}{A_{0-1}}=\left(\frac{1-0.96 J_{0} / w_{0}}{1+0.292 J_{0} / w_{0}}\right)
$$


Figure 2. Calculated absorption (blue) and emission (red) spectra for linear H- and J-aggregates containing $N=20$ molecules with nearest-neighbor-only coupling. In all calculations the value of $\omega_{0}=0.17 \mathrm{eV}\left(1400 \mathrm{~cm}^{-1}\right)$ were taken. The exciton bandwidth, $W=4\left|J_{0}\right|$, increases from top to bottom [(a-e) for H-aggregate and (f-j) for J-aggregate]. Solid spectra are evaluated using one- and two-particle states, while dashed spectra use only one-particle states. Inset in panel (a) shows the isolated-molecule spectra. Gray absorption spectrum appearing in all panels pertains to the isolated molecule $(W=0)$. Insets in panels (d) and (e) are enlarged emission spectra. Inset in panel (j) shows unscaled emission spectrum for different values of $N$ (Reprinted with permission from [32]. Copyright 2010 American Chemical Society).
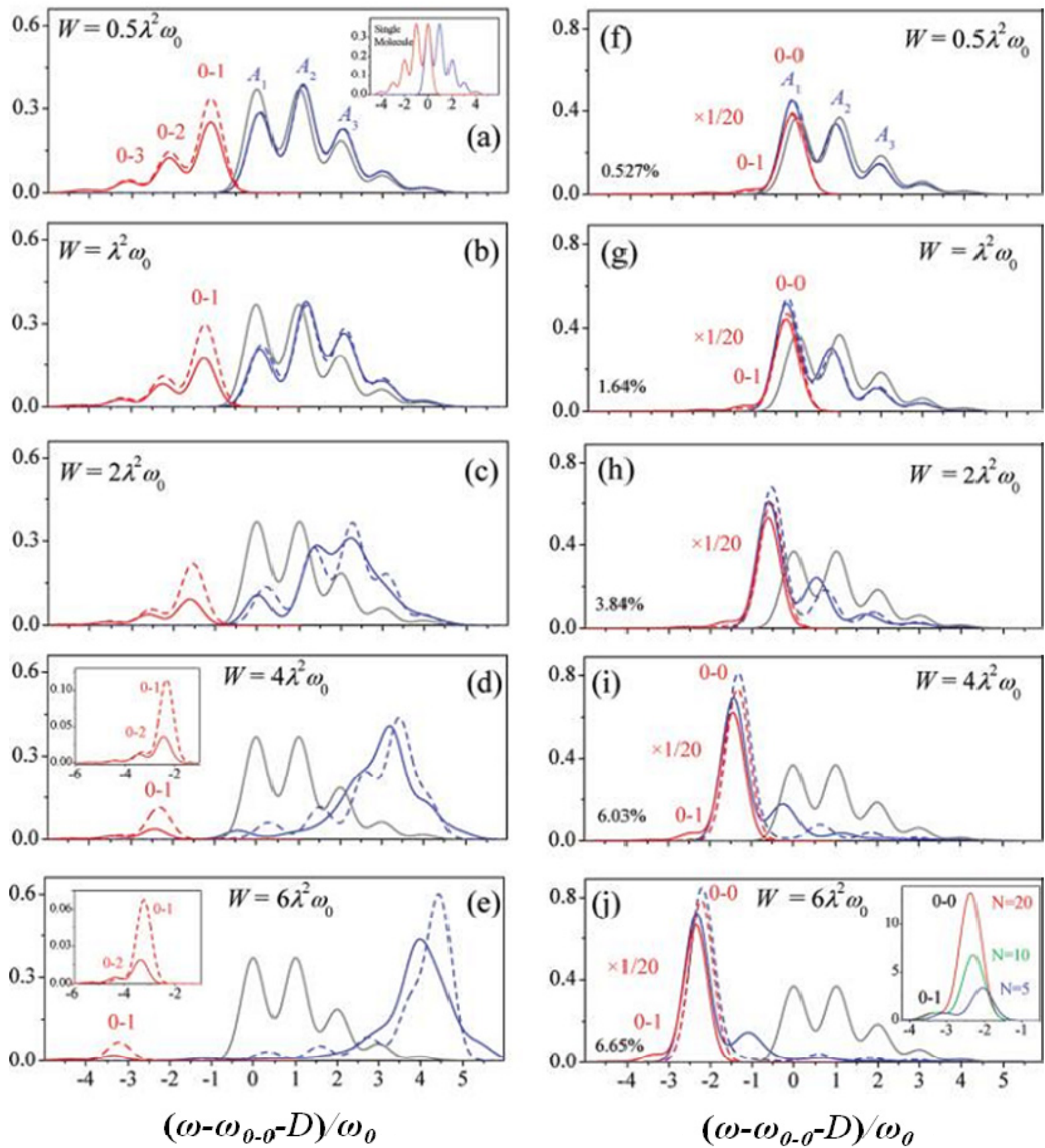
In Equation (1), $J_{0}$ and $\omega_{0}$ are the nearest neighboring coupling and the frequency of nuclear potentials equivalent to shifted harmonic wells, respectively. The value of $J_{0}$ is positive for $\mathrm{H}$-aggregation $\left(R_{\mathrm{abs}}<1\right)$ and negative for J-aggregation $\left(R_{\mathrm{abs}}>1\right)$. The free exciton bandwidth $W$ as an important parameter describing the conjugation length in chains is defined as $W=4\left|J_{0}\right|$. According to the results in Figure 2, it is found the J-aggregation has a better stability and shows a better structural order beneficial for charge transport in terms of electronic energy than $\mathrm{H}$-aggregation with the same $W$. Meanwhile, with the decreasing $W$ for $\mathrm{H}$-aggregation and increasing $W$ for J-aggregation, the order of aggregation is improved indicated by the red-shifted spectra and enhanced $R_{\text {abs }}$ as mentioned above. Along with the electronic transitions, the interaction mode of rod-shaped molecules also has an impact on molecular aggregation type: a pair of rod-shaped molecules in a "side-by-side" orientation forms the so-called H-aggregates [33,34] and a pair in a "head-to-tail" orientations forms the so-called J-aggregates [35]. For conjugated polymers with planar backbones and rod-like conformations, it is hoped that the conjugation could be extended to the maximal extent along the backbone plane, which could lead to a more ordered J-aggregation beneficial for both its continuous transport of charge carriers and its better inter-chain stacking, or an improved inter-chain stacking in H-aggregation. All these improvements could be reflected by the red-shifted absorption spectra and/or the increased value of $A_{0-0} / A_{0-1}$. Fortunately, the J-aggregation has been found in poly(3-hexylthiophene) (P3HT) [36] and many donor-acceptor copolymers with extended intra-chain conjugation and a good planarity in their backbones [37-39]. By strategies such as choosing processing solvent with a high boiling point [31], dispersing the nanofibrils [40] and thermal annealing [38,39], etc., the more ordered aggregation in films is strengthened corresponding to the red-shifted absorption, enhanced $A_{0-0} / A_{0-1}$ value, more ordered fibrillar morphology and improved mobility. Thus, it is concluded that a improved crystalline order and/or aggregation order with good intra-chain conjugation and inter-chain stacking in crystallites is helpful to obtain more ordered morphology and higher electronic performances.

\subsection{Connectivity between Crystalline Regions}

Due to the polycrystalline nature of most conjugated polymer films, the connectivity between adjacent crystalline regions becomes a barrier for charge transport because the original pathways are interrupted (Figure 1b,c). Therefore, the grain boundary become the bottleneck for improving mobility in terms of (1) the density of grain boundary; (2) the barrier of grain boundary; and (3) the bending angle in grain boundary. Firstly, the stacking mode of adjacent crystalline regions along the apparent transport direction has an effect on the grain density and grain barrier [41,42]. Jimison et al. [41] and Lee et al. [42] prepared anisotropic films of P3HT and pBTTT by directional crystallization and zone-casting, respectively. For both polymers, the mobility along the backbone direction was much higher than that along $\pi-\pi$ stacking direction. By morphological analysis and mobility-temperature measurement, it was found that the density of grain density and the barrier height for charge transport along backbone direction were both smaller that those along $\pi-\pi$ stacking direction. Thus, intra-chain transport and connection between adjacent crystalline regions in an "end-to-end" mode are thought beneficial for efficient charge transport. In contrast, the connection of "face-to-face", side-chains, different oriented crystalline regions and disordered regions are all larger barriers for hopping of carriers (Figure 3I). Secondly, the tie chains connecting adjacent crystalline regions (Figure 1b) play an 
important role in decrease the transport barrier of carriers at grain boundary [43-45]. As known, the disordered regions between adjacent ordered domains limit the efficient transport of carriers. Lan et al. reported that there were three modes for chains in disordered regions between adjacent crystalline regions: extending, looping, and bridging chains [43]. In the disordered domains, the charge transport can occur in the interchain pathway by hopping between close enough chain ends/loops or in the intrachain pathway along the bridging chains. The significant difference of the charge mobility through the end/loops and bridging chains, and the ratio of their amount is crucial to determine the average charge mobility in disordered regions. In fact, the experimentally measured charge mobility is limited to a maximum of $10^{-2} \mathrm{~cm}^{2} \cdot \mathrm{V}^{-1} \cdot \mathrm{s}^{-1}$ due to the presence of more end/loops instead of bridging chains in disordered regions. Kline et al. reported that for P3HT with a higher molecular weight the transport barrier was smaller (54 meV) than that of P3HT with lower molecular weight $(>60 \mathrm{meV})$ [44]. They attributed it to that the long chains can interconnect ordered areas and prevent charge carriers from being trapped by the disordered boundary regions by creating a continuous pathway through the film. Accordingly, the films spin-coated from P3HT with a high molecular weight displayed better mobility than that of lower molecular weights. For P3HT films made of nanofibril webs, Bolsee et al. reported that the electrical resistance associated with the bridging of two or more individual nanofibrils did not reduce the charge transport inside the web of nanofibrils by observing the current maps in detail [45]. They found that the electrical resistance of a bridging point, $R_{\mathrm{bp}}$, was far smaller than that of the whole nanofibrils, $R_{\mathrm{NF}}$, that is, $R_{\mathrm{bp}}<0.1 R_{\mathrm{NF}}$. They also attributed it to the tie chains connecting the two crossing nanofibrils. Thirdly, the relative orientation between transport direction in adjacent crystalline regions has an effect on the effective transport of carriers [46-49]. Street et al. pointed out that in plane the nanocrystalline lamella had specific structural orientations defined by the stacking directions of their chains, and the electronic property of the boundary between two adjacent grains depended primarily on their relative orientations, in other words, the angle of grain boundary [46]. The grain boundaries are not isotropic, and there are favorable and unfavorable transport directions across the boundaries. Small angle grain boundaries for lamella are connected end-to-end with respect to the chain orientation, which will exhibit some interpenetration of the chains in the boundary. In spite of considerable disorder, side-to-side grains also have the possibility of a reasonably smooth connection at some locations, where the links between grains are bent chains of low bend angles. For high-angle grain boundaries, they are made of bent chains with a higher bend angle and therefore more disordered. The charge transport is dependent on the bent chains and the correlation between the localization energy and the bend angle. A percolation path along preferential directions with small-angle boundaries is beneficial for efficient charge transport, which has been proven in examples of pBTTT films $[46,48,49]$. Zhang et al. proved that in annealed pBTTT films the boundaries between adjacent liquid-crystal-like domains with different crystal orientations were small-angle ones utilizing dark-field transmission electron microscope (TEM) [48]. As high as 95\% of neighboring regions for a domain with a minor orientation and $68 \%$ for that with a major orientation were made of domains with most similar orientations to it, which was thought to be a structural origin of the high carrier mobility of annealed pBTTT films (Figure 3II). Schuettfort et al. pointed out that the liquid crystalline declinations with high-angle grain boundaries were sites of low order in the pBTTT films with different morphologies employing scanning transmission X-ray microscopy (STXM) technology [49]. For example, in zone-casting pBTTT films reported by Lee et al. [42], the larger transport barrier of 
grain boundary perpendicular to backbone direction (Figure 3I) was attributed to the nearly $90^{\circ}$ degrees declination between adjacent domains (Figure 3II) [49]. Thus, small-angle grain boundaries are wanted for a better charge transport along the percolation pathways despite the inevitability of grain boundaries. In summary, end-to-end connection of adjacent crystalline regions, a larger amount of tie chains in grain boundaries and small-angle grain boundaries along transport pathways are three important factors to improve the connectivity between adjacent crystalline domains.

Figure 3. (I) Illustrations of possible grain boundaries in directionally crystallized thin films. The first panel depicts grain boundaries most relevant in parallel devices, showing chains that bridge between (a) two edge-on grains and (b) two face-on grains; The second panel consists of grain boundaries more relevant to the perpendicular devices, between (c) two edge-on grains; (d) two face-on grains; (e) one face on and one edge-on grain; and (f) two edge-on grains, with a disordered region between crystallites (gray area) in (A) (Reprinted with permission from [41]. Copyright 2009 Wiley-VCH Verlag); SEM image of ribbon-phase PBTTT film in (B); schematic diagram of charge transport in ribbon-phase PBTTT films with the red lines schematically indicating the individual polymer chains in (B') and temperature dependence of the linear field-effect mobility of uniaxially aligned ribbon-phase PBTTT for transport parallel and perpendicular to the backbone direction in (B'), (Adapted with permission from [42]. Copyright 2011 Wiley-VCH Verlag); (II) Orientation maps of annealed pBTTT spin-coating films in (A) and neighborhood analysis curves for minor (A') and major (A' $\left.\mathbf{A}^{\prime \prime}\right)$ colored pixels (Adapted with permission from [48]. Copyright 2010 Wiley-VCH Verlag); and atomic force microscope image and orientation maps for terrace and aligned nanoribbon PBTTT films in (B) with two nearby pairs of half integer declinations circled in red and black in terrace films and the inset shows a magnified drawing of the orientation (black) and backbone directions (red) in ribbon films (Adapted with permission from [49]. Copyright 2012 American Chemical Society).
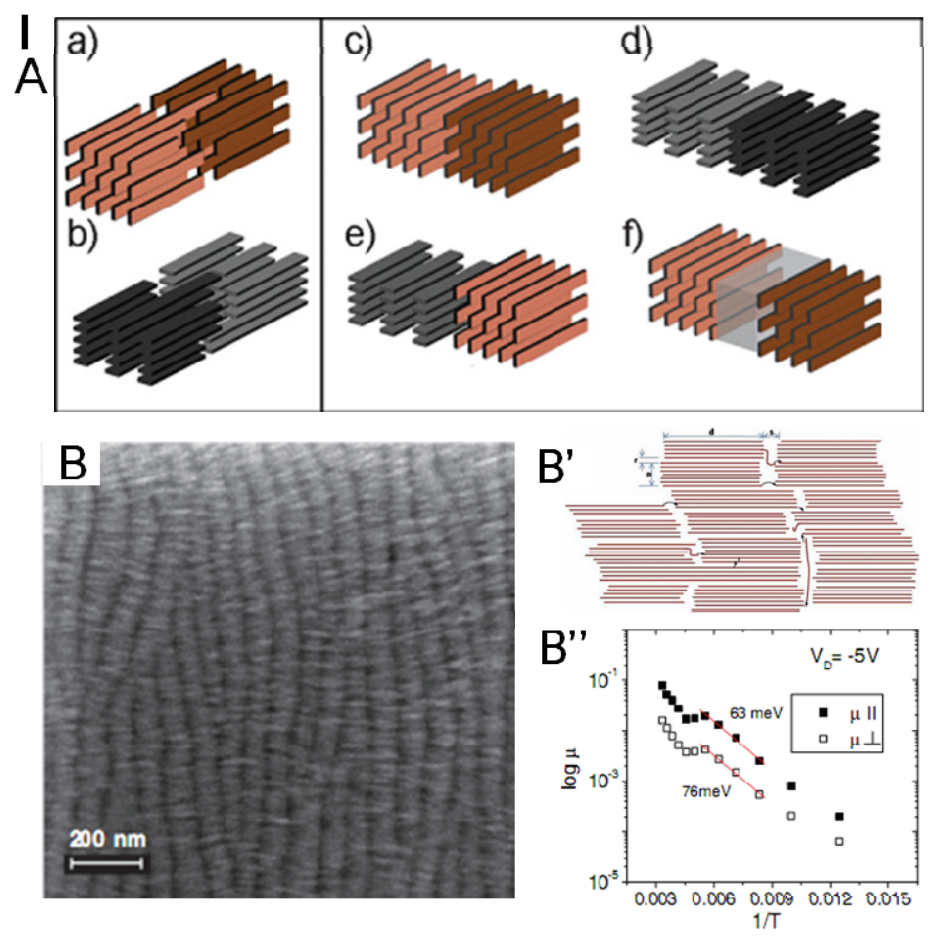
Figure 3. Cont.

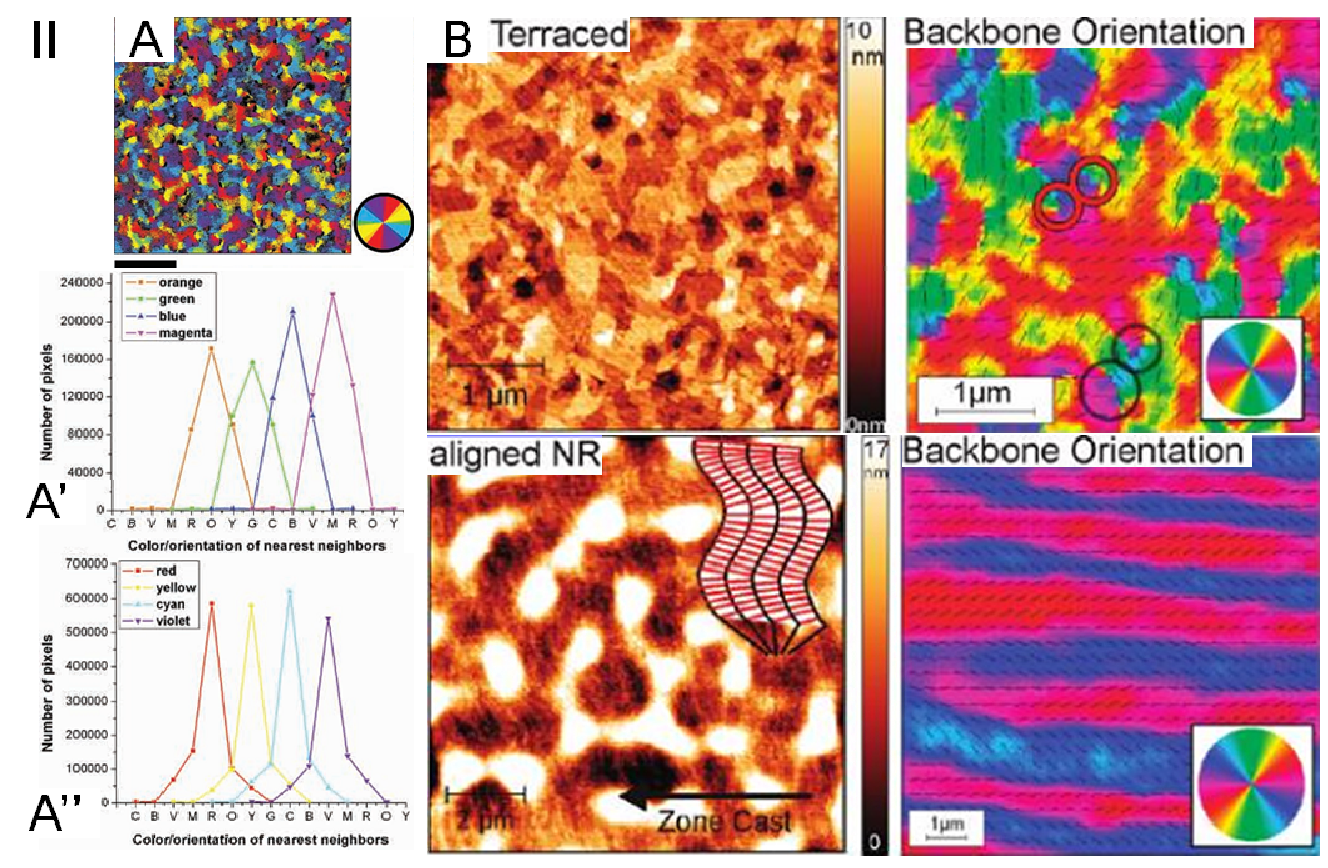

\subsection{Crystallinity and Crystallite Alignment}

As an intrinsic property rather than doping, the amount of crystalline regions in films also plays an important role in the transport of charge carriers via determining the amount of transport pathways in the scale of devices for (semi-) crystalline conjugated polymers which contribute a large proportion in the catalogue of conjugated polymers, although several amorphous conjugated polymers such as dithieno[3,2-b:2',3'-d]pyrrole(DTP)-co-thiophene(TH)s and poly[3-(5-((2,6-bis((4-hexylphenyl)ethynyl)-10-(prop-1-yn-1-yl)anthracen-9-yl)ethynyl)thiophen-2-yl)-6-(5-methylthi-ophen-2-yl)-2,5-bis (2-octyldodecyl)py-rrolo[3,4-c] pyrrole-1,4(2H,5H)-dione] also have high carrier mobilities [50,51]. More crystallites in films means more efficient pathways for carriers, and fewer disordered regions and/or grain boundaries limiting mobility in films. Thus, a increase of crystallinity in films could enhance the carrier mobility in films [38,52-54]. The increase of crystallinity is often reflected in terms of (1) enhanced absorbance attributed to ordered aggregation of chains in UV-Vis spectra [53]; (2) the higher diffraction peaks in X-ray diffraction (XRD) profiles [38,52,54] and (3) more obvious and/or ordered crystalline morphology in films such as nanofibrils [52,53], etc. Aiyar et al. reported that ultrasonic vibration could increase the ordered aggregation in solution and the crystallinity in films [52]. With increasing vibration time, the $0-0$ absorbance in solution and films and the (100) peak height increased until reaching a platform after vibrating for $5 \mathrm{~min}$, accompanied by the development of fibrillar morphology of films. Correspondingly, the carrier mobility in films was enhanced with the increasing crystallinity and reached a maximum at last. For donor-acceptor copolymers, the increasing crystallinity reflected in enhanced (100) peak height in XRD profiles corresponds well to a better hole mobility with a higher annealing temperature [38,54]. The enhanced crystallinity is also helpful to improve the performance of polymer/fullerene solar cells [53]. When enhancing the sonication temperature of P3HT toluene solution, the $0-1$ and $0-0$ absorbance in solution gradually increased. As a retention of aggregation in solution, the amount and length of nanofibrils increased obviously 
reflecting improved crystallinity in films. More long nanofibrils tended to connect each other well and form homogeneous network, which provided good percolation pathways for holes not only in pure P3HT films but also in P3HT/[6,6]-phenyl- $\mathrm{C}_{61 / 71}$-butyric acid methyl ester (PCBM) blend films. As known, improved charge transport is an important factor to enhance the conversion efficiency of solar cells. Therefore, the devices made of nanofibrils with the larger amount and the biggest length $(560 \mathrm{~nm})$ showed the best performance $(2.32 \%)$ than those of few and short nanofibrils (135 nm, $1.93 \%$ ). Thus, increasing the crystallinity is an important strategy to improve the morphology and electronic properties of conjugated polymer films. For those amorphous conjugated polymers with high carrier mobilities, the origin was attributed to the presence of long arrange $\pi$-conjugated pathways in microscopic scale [50] or the low bandgap combined with highly connected and compact structures indicated by a low surface roughness [51], as mentioned in Section 2.2.

Another ideal structure in films is all the charge transport pathways are straight from source to drain and from cathode (anode) to anode (cathode) for FETs and OPVs, respectively (Figure 1c). For OPVs requiring the charge transport normal to substrates, the ideal alignment of crystallites equals to the realization of face-on [55,56] or flat-on orientation [57] of conjugated planes respect to substrates, in other words, to align the efficient intrachain pathways or interchain $\pi-\pi$ pathways vertical to substrates. For conjugated polymers adopting preferential edge-on orientation in films, epitaxy crystallization assisted by 1,3,5-trichlorobenzene (TCB) [41,58], mechanic rubbing at a certain temperature [59] and adding additives such as diiodooctane (DIO) [55] are three effective methods to promote face-on orientation in films. Meanwhile, slow evaporation of solution in $\mathrm{CS}_{2}$ [60], $\mathrm{CS}_{2}$ vapor annealing [61], vapor-assisted imprinting [57] and thermal nano-imprinting [62] have been employed to acquire flat-on molecular orientations in P3AT films. For FETs, although some donor-acceptor copolymers such as poly(diketo pyrrolo-pyrrole-co-thiophene) (PDPP-T) and poly\{[N,N9-bis(2-octyldodecyl)-naphthalene1,4,5,8-bis(dicarboximide)-2,6-diyl]-alt-5,59-(2,29-bithiophene)\} [P(NDI2OD-T2)] display high mobility in spite of a mainly face-on orientation $[37,63]$, the edge-on molecular orientation of chains respect to substrates is favored for most conjugated polymers because of the in-plane $\pi-\pi$ pathways wanted by device architecture. The devices with edge-on orientations have a better mobility than those with face-on orientations in examples like P3HT and pBTTT et al. [59,64,65]. For high-mobility face-on devices, the origin may be that carriers can successfully overcome barrier near dielectric interface by hopping to at least subsequent $2-3$ layers coupled by out-of-plane $\pi-\pi$ interaction to achieve a three-dimensional transport [63].

Another challenge for FETs lies in the in-plane alignment of transport pathways in FETs. The ideal situation in FETs is that all the efficient transport pathways are straight from source to drain with hardly directional deviation. Wang et al. prepared aligned structures in films by dip-coating and proved that films made of aligned nanofibrils connecting source and drain displayed outstanding good carrier mobility of FETs in the examples of pBTTT (Figure 4) and P(NDI2OD-T2) [66,67]. The fibrillar film with a long-range alignment had a much higher mobility $\left[0.7 \mathrm{~cm}^{2} \cdot \mathrm{V}^{-1} \cdot \mathrm{s}^{-1}\right.$ for $\mathrm{pBTTT}$ and $0.043 \mathrm{~cm}^{2} \cdot \mathrm{V}^{-1} \cdot \mathrm{s}^{-1}$ for P(NDI2OD-T2)] than that with random orientations $\left[0.36 \mathrm{~cm}^{2} \cdot \mathrm{V}^{-1} \cdot \mathrm{s}^{-1}\right.$ for pBTTT and $0.025 \mathrm{~cm}^{2} \cdot \mathrm{V}^{-1} \cdot \mathrm{s}^{-1}$ for P(NDI2OD-T2)]. Besides, the transport pathways aligned between source and drain should be efficient ones with fewer and softer grain boundaries. In aligned films free of nanofibrils such as P3HT and pBTTT, the efficient pathways were along backbones for their lower 
grain boundary density and smaller barrier [41,42]. Therefore, to align pathways preferred for efficient transport between source and drain is very crucial to improve the performance of FETs.

Figure 4. The pBTTT films with (a-c) random orientations and (d) a good alignment prepared via controlling dip-coating rates (Adapted with permission from [66]. Copyright 2012 American Chemical Society).

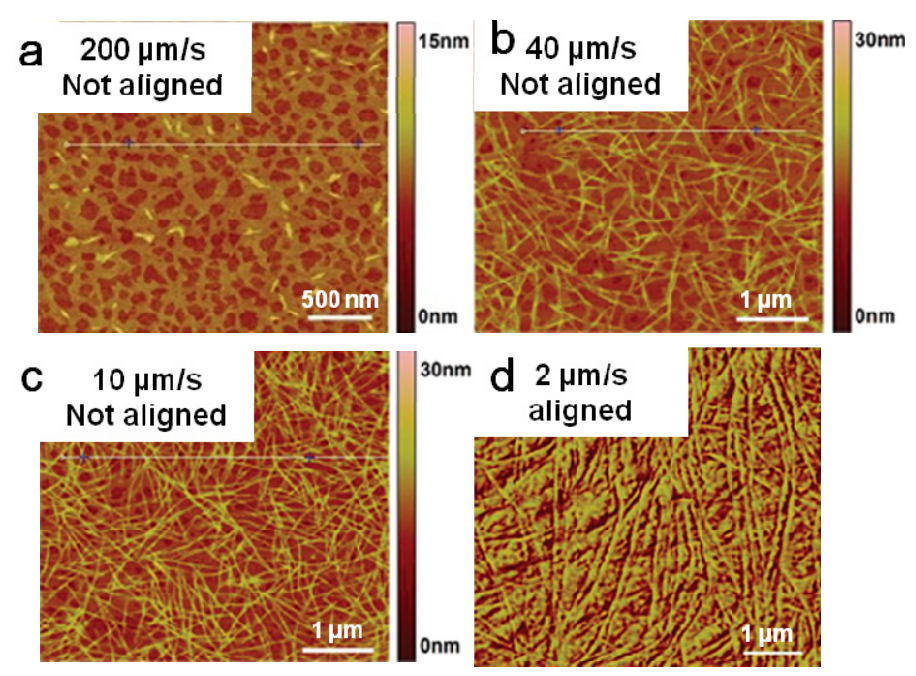

To summarize this section, morphological and structural features such as (1) a good internal order of crystalline region; (2) a good connectivity between adjacent crystalline regions in end-to-end mode with small-angle grain boundaries and more tie chains and (3) a higher crystallinity and better alignment of preferred transport pathways between electrodes are the targets to obtain better device performances. These morphological and structural features are closely related with (1) intrinsic molecular structure of conjugated polymers and (2) the state of chains in solution and proper solution-processing strategies. Thus, the influences of them on morphology and structure of conjugated polymer films will be discussed in following.

\section{The Effects of Conjugation Structures of Semiconducting Polymers on Their Electronic Properties, Structures and Morphology}

There is no doubt that the conjugation in backbones is a determining factor for all optical and electronic properties of semiconductor conjugated polymers, for the delocalization of electrons, in other words, $\pi$-conjugation in and between backbone planes provides transport pathways of carriers. Meanwhile, the morphological and structural features also depend heavily on the intrinsic conjugation structure of molecule chains such as planarity and rigidity of backbones, chain shapes, molecular weight and side-chain distribution, etc., which influence the performances in turn. In following, the characteristic structural features of conjugated polymer compared with flexible chains will be discussed first. Then, the effect of molecular structural features on structure, morphology and charge transport will be discussed in detail. 


\subsection{Structural Differences between Conjugated Polymers and Flexible Polymers and the} Corresponding Effects on Morphology

Compared with flexible linear polymers such as polystyrene (PS), the conjugation of $\pi$-electrons leads to much enhanced rigidity of chains. It is known that a polymer chain could be regarded as its equivalent Kuhn chains, in which the motion units are modeled as Kuhn segments made of several repeating units $[68,69]$. Due to the large bending barrier of stiff conjugated polymers, the Gaussian chain model fitting well with flexible chains [Equation (2)] is replaced by worm-like chain model [Equation (3)] to better depict the conformation of rigid conjugated polymers,

$$
\begin{gathered}
\left\langle R_{\mathrm{e}}{ }^{2}\right\rangle=N b^{2}, l_{\mathrm{K}}=b \\
\left\langle R_{\mathrm{e}}{ }^{2}\right\rangle=N b^{2} \frac{1+c}{1-c}, l_{\mathrm{K}}=b \frac{1+c}{1-c}, c \approx 1-k_{\mathrm{B}} T / \varepsilon, \xi=\frac{b}{\ln (1 / c)}
\end{gathered}
$$

where $\left\langle R_{\mathrm{e}}{ }^{2}>, N, l_{\mathrm{K}}, \varepsilon\right.$ and $\xi$ are end-to-end distance, number of segments, length of Kuhn segment, energy cost associated with stiffness and persistence length, respectively. The extension of conjugation plane in backbones arising from huge $\pi$-systems makes the persistence length of chains elongated, leading to a much longer Kuhn segment length and extended conformation of chains than those of flexible polymers especially in solutions. The conformational change brought about by conjugation structure results in different structural and morphological features from flexible chains. Firstly, the elongated persistence length of chains of conjugated polymers makes their single chains display a more anisotropic and extended conformation with less bending like paperclips, compared to the isotropic and disordered coils of more flexible chains (Figure 5a) [69]. Zhao et al. proved that the persistence length of pBTTT $(9.0 \mathrm{~nm})$ was much larger than $\mathrm{rr}-\mathrm{P} 3 \mathrm{HT}(4.8 \mathrm{~nm})$ due to the introduction of fused thieno[3,2-b]thiophene moiety into backbones to strengthen the conjugation, implying more rigid chains than flexible chains like PS $(2.8 \mathrm{~nm})$ [68]. These conjugated polymer chains display more rod-like conformations, which tend to crystallize driven by intense $\pi-\pi$ interaction of conjugation planes different from flexible chains. Therefore, 1-D aggregates like nanofibrils with large aspect ratios are frequently formed in films for anisotropic conjugated polymers, unlike 2-D aggregates such as spherulites or lamella single crystals of polyethylene (PE). Secondly, the chain folding in conjugated polymers is also different from those in flexible polymers. In conjugated polymers, a more extended chain-folding exists, i.e., seven thiophene rings in the folding section in P3HT and nine for P3DDT (Figure 5b) [70]. Similar to flexible chains, the chain-folding could drive the crystallization of chains. The folded chain model and fringed micelle model proposed for crystalline or semi-crystalline flexible polymers have been also applied in the examples of conjugated polymer polycrystalline films to explain crystal formation, tie chains and amorphous regions. As to the lamellar structure [71], the bending of chains near the ends gave rise to disorder region in lamella. With the increasing molecular weight, the total lamellar thickness increased but the size of crystalline region was nearly unchanged. Fortunately, tie chains connecting adjacent lamella were observed in thickened disorder region by HR-TEM for high molecular weight P3HT (Figure 6I). As to crystal formation, the chain-folding is controlled by the planarity of conjugated polymers, which plays a crucial role in determining the size of crystalline structures. As to dispersed nanowires, the width was determined by the planarity and rigidity of chains as reported by Liu et al. [72]. They proposed that the change of nanowire width with 
increasing molecular weight depended on the inter-chain torsion barrier in chains (Figure 6II). The large barrier for torsion of $\mathrm{C}-\mathrm{C}$ single bond in dithiophene, tail-to-tail linked dialkylthiophoene and alkylthiophene-thiazolo[5,4- $d]$ thiazole moieties resulted in the non-folding nature of PQT and PTzQT chains. Thus, the contour length of PQT and PTzQT chains equaled to the width of nanowires. For P3HT and pBTTT, the barrier was small and the width of nanowire reached a maximum when molecular weight excessed a critical value. Besides, compared to the single crystals of flexible polymers like PE with chain-folding obtained from dilute solution relatively simply, those of conjugated polymers are made of extended chains without folding [73,74]. Due to the difficulty of unfolding chains to a totally extended conformation, there are few examples of preparing conjugated polymer single crystals under strict conditions like concise temperatures, extremely slow evaporation and/or specific substrates, etc. [73-78]. Thirdly, according to Einstein-Stokes Equation, $D=K_{\mathrm{B}} T / 6 \pi \eta a$, the diffusion coefficient $(D)$ is inverse to the sizes of moving unit $(a)$ in solutions. Thus, the motion of segments and the whole chains of conjugated polymers is more hindered due to bigger segments and more extended conformations, which affects the effective approaching and stacking of chains. It brings difficulty to the crystallization of chains during casting or post-annealing. Especially for conjugated polymers with larger conjugation lengths, Wang et al. reported that (1) the critical nucleation vapor pressure was higher due to the higher critical vapor pressure for rod-to-coil transition during solvation and (2) the fibril density was reduced partly due to the slower motion of units during aggregation, which were the results of the larger conjugation lengths and enhanced rigidity for polymers (Figure 7) [79]. Therefore, tuning the crystallization and morphology of conjugated polymers is challenging due to their conjugated molecular structures. In the following, the effects of structural factors on the structures, morphology and charge transport in films will be discussed.

Figure 5. (a) The conformations of regio-regular P3HT (rr-P3HT) and regio-random P3HT (rra-P3HT) shown omitting side-chains, (Adapted with permission from [69]. Copyright 2011 American Chemical Society) and (b) the STM images of P3HT and P3DDT chains on HOPG surface with the corresponding schematic illustration of chain packing and chain-folding, (Adapted with permission from [70]. Copyright 2000 Wiley-VCH Verlag).
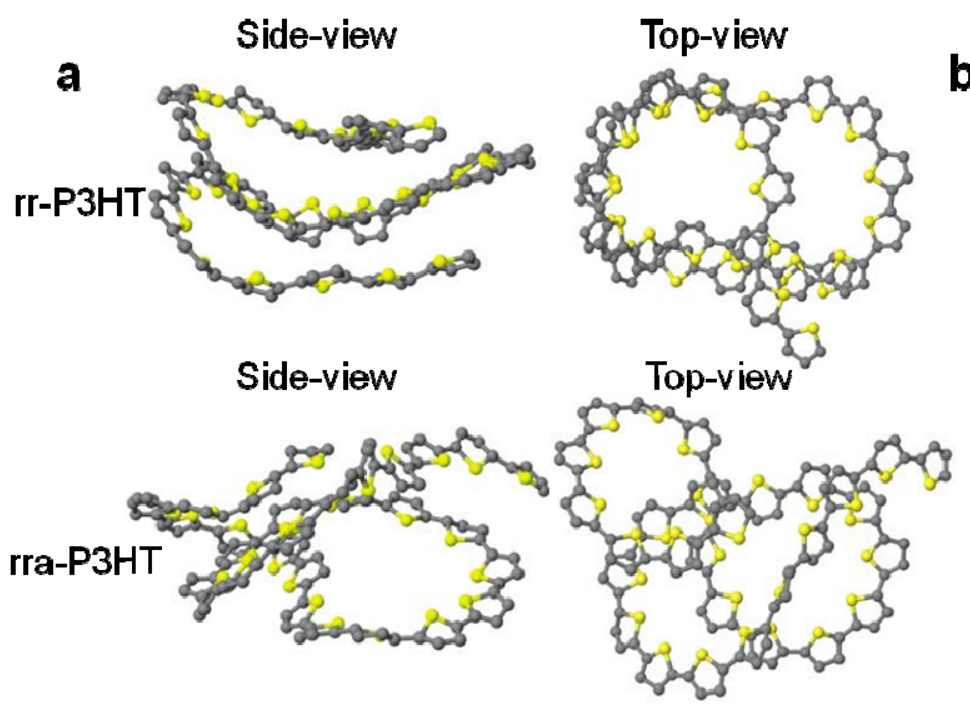

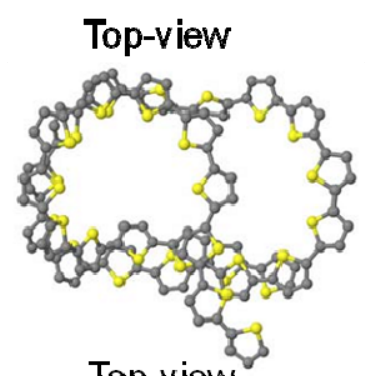

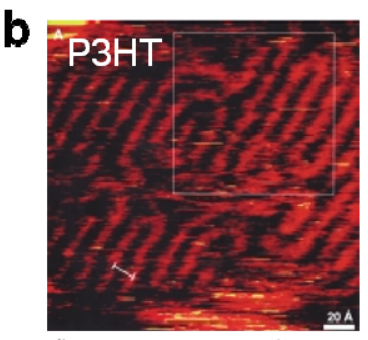

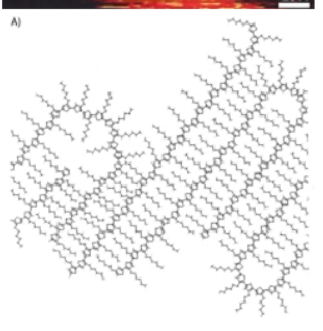

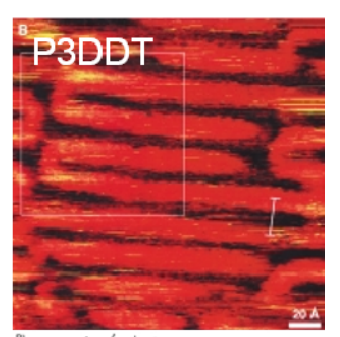

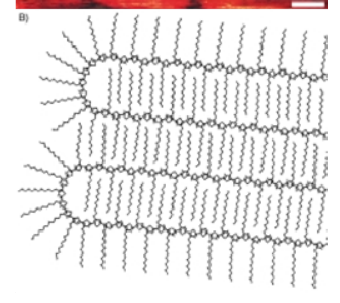


Figure 6. (I) Schematic representation of the microstructure size in semicrystalline Rr-P3HT of different molecular weights influenced by chain-bendings and the HR-TEM photos for bendings indicated by the arrow, (Adapted with permission from [71]. Copyright 2009 American Chemical Society); (II) Dependence of average nanowire width on average contour length of PQT-12, PBTTT-14, PTzQT-14, and rr-P3HT in (A) and simplified cartoons illustrating the difference between conjugated polymer nanowires with folding (bottom) and nonfolding (top) chains in (B) and TEM photos for nanowires of PQT12, pBTTT and pTzQT with similar high molecular weights in (C), (Adapted with permission from [72]. Copyright 2011 Elsevier).
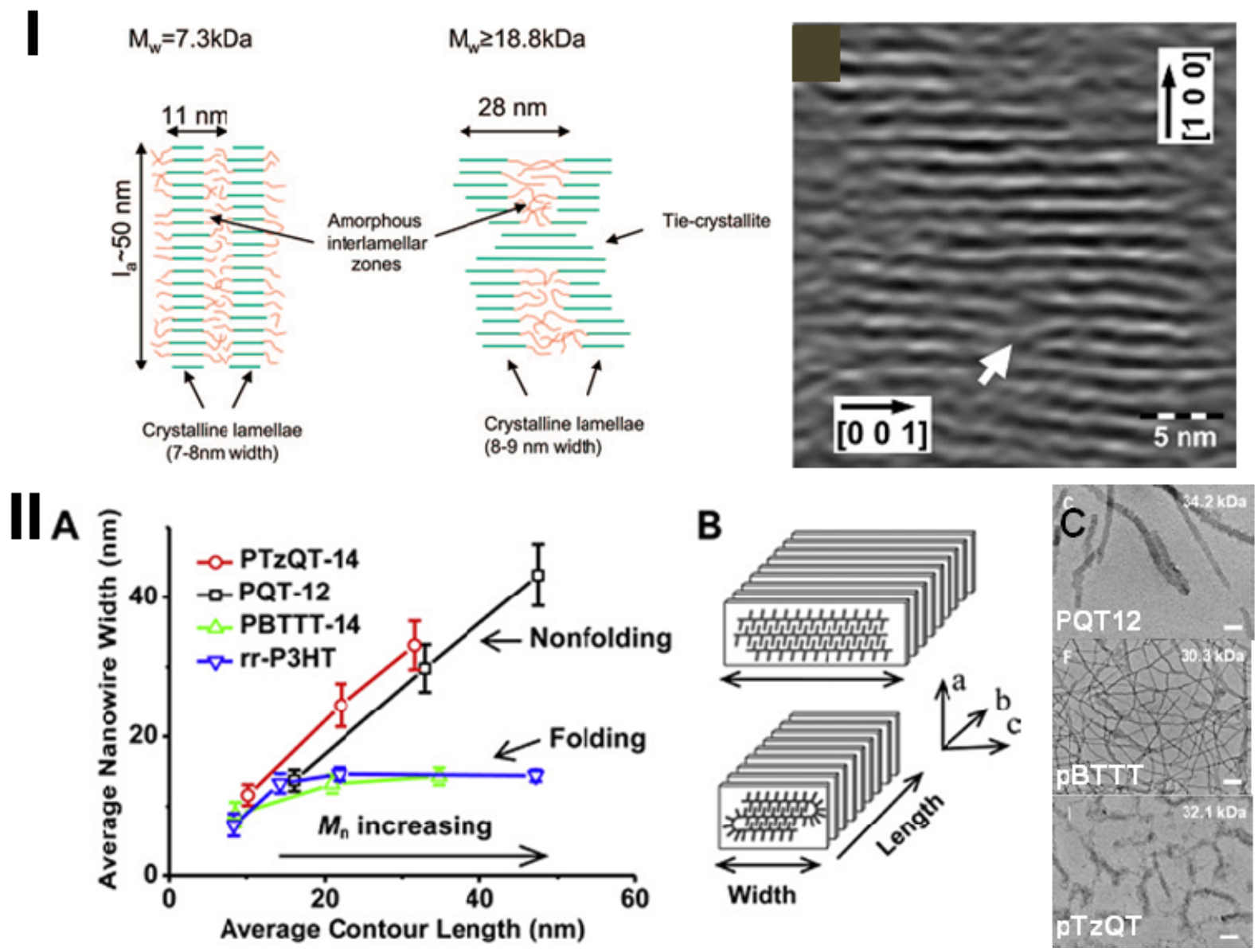
Figure 7. The TEM images of films annealed at $P_{\mathrm{CS} 2}=98.3 \%$ with a increasing conjugation length from left to right and scale bars of $500 \mathrm{~nm}$ in (a), the variation of free exciton bandwidth $\mathrm{W}$ with $\mathrm{P}$ indicating the conformation change in (b) and dynamic light scattering (DLS) results of polymers in solution in (c) (Adapted with permission from [79]. Copyright 2013 American Chemical Society).
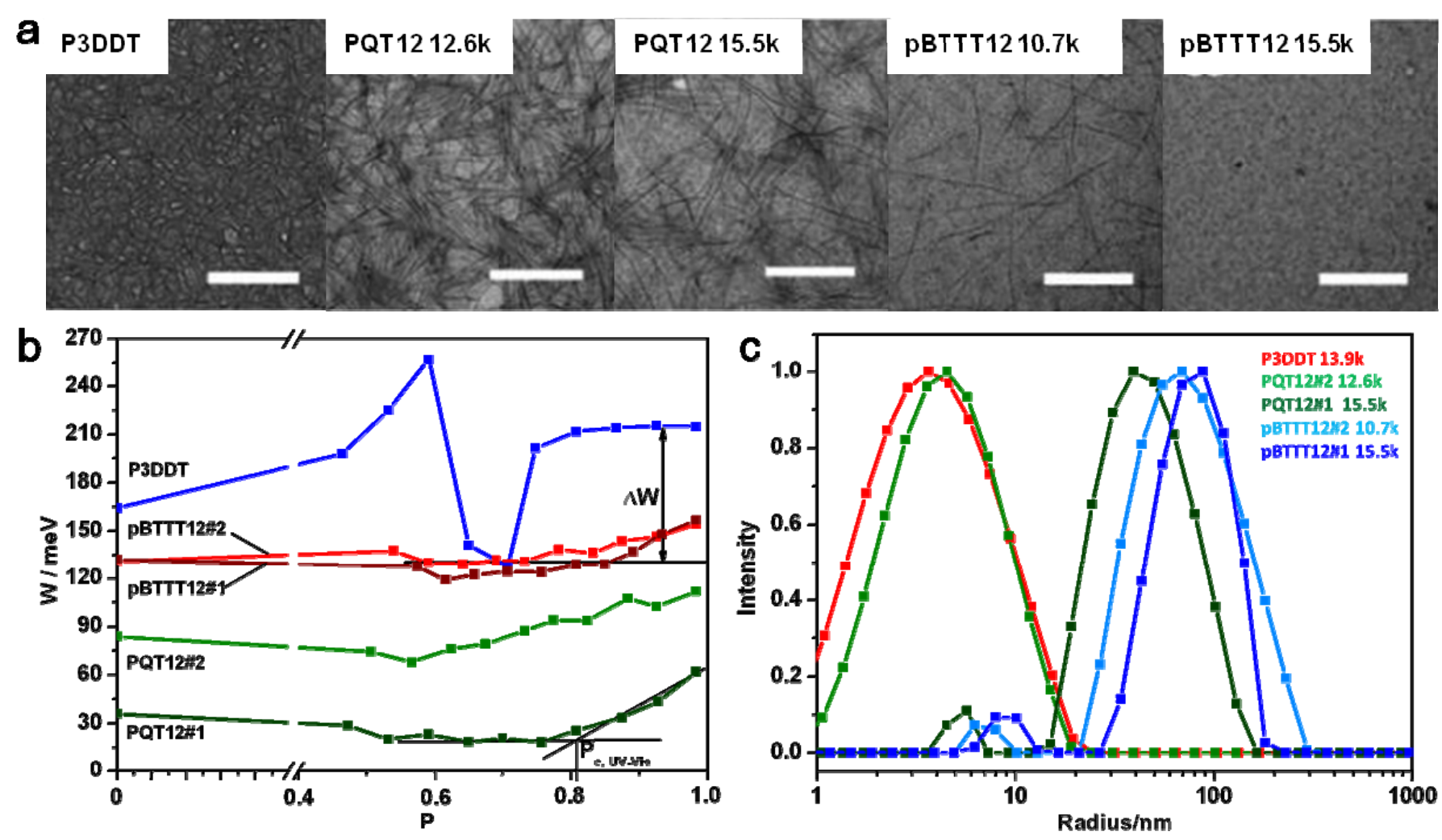

\subsection{The Effect of Planarity and Rigidity on Structure, Morphology and Charge Transport}

As the direct reflection of conjugation in backbones, the planarity is defined as the extent of conjugated moieties in backbones being in the same plane indicated by inter-ring torsion dihedral angle which plays an important role in the charge transport. In charge transport theories, the mobility is determined by two quantum mechanics parameters, transfer integral and reorganization energy. The transfer integral of holes (electrons) could be obtained from the difference between energy levels of HOMO and HOMO-1 (LUMO and LUMO+1) molecular orbitals [1,43]. A larger transfer integral leads to a better hole (electron) mobility. The mobility of carriers through two adjacent rings with a torsion of $\theta$ is proportional to $\cos ^{2} \theta$ [80], which means that transfer integral and mobility are smaller for a larger inter-ring torsion angle (0-90 degrees) [43]. Due to the small barrier, a small torsion angle of 5-10 degrees could transform easily in the work environment of devices to 0 (180) degrees, that is, a perfect planar structure. Therefore, a small inter-ring torsion (better planarity) is beneficial for charge transport. Another parameter is reorganization energy defined as the energy loss when a charge carrier passes through a conjugated unit. The smaller the organization energy is, the larger mobility of carriers is. A more planar conjugation structure is helpful to extend the conjugation length, strengthen the 
conjugation intensity, decrease the reorganization energy and enhance carrier mobility and/or OPV efficiency, as proved both in theory and experiments [1,38,81].

Considering the importance of intrinsic conjugation structures on electronic properties and conformation mentioned above, it is necessary to understand it in terms of molecular design for rational synthesis and chose of materials in applications. Some conjugated polymers with out-standing carrier mobilities are listed in Table 1 to demonstrate the effects of molecular structures on their electronic properties. Firstly, extending the conjugation system and strengthening intra-chain and inter-chain interaction is a direct way to improve electronic properties. Based on the classic conjugated polymers such as P3ATs (P1), a straight strategy is inserting more conjugated moieties $[3,82]$ such as carbon double bond $(-\mathrm{HC}=\mathrm{CH}-$, P2) [83-85], unsubstituted multiple or fused aromatic rings (i.e., thiophenes and thieno[3,2-b]thiophene, etc., P3-P7) [13,14,16,37,82,86-91], other heterocyclic rings containing nitrogen(and sulfur) (P8 and P9) or selenium atoms (P10) [13,15,81,92-97] and their combination (P11) [98]. Thus, the intra-chain conjugation is extended and strengthened by coupling with the more conjugated moieties, and the conjugation length is elongated due to the improved planarity. As a result, the inter-chain interaction of these extended conjugated chains becomes more intense to form more stable and ordered aggregates, resulting in the enhanced performance of devices [81]. Secondly, inserting acceptor moieties as n-type units [i.e., diketopyrrolopyrrole (DPP), naphthalene-bis(dicarboximide) (NDI), etc.] into p-type moieties has become a very efficient strategy to synthesize more conjugated polymers by employing the intense intra- and inter- chain donor-acceptor interactions recently $[11,86,99-103]$. Take DPP unit for an example, the combination of DPP and thiophene, that is, dithienyl-DPPs, show excellent planarity with a small torsion of only 12 degrees due to both the intense donor-acceptor interaction via electron transfer and the favorable intra-molecular S-O interaction [11]. Many high-mobility copolymers based on dithienyl-DPPs have been synthesized through coupling with intensely conjugated p-type moieties such as thieno[3,2- $b]$ thiophene, unsubstituted dithiophene, diselenophene, benzothiadiazole, etc. (P12-P16) [11,37,104-106]. The conjugated polymer with extremely high carrier mobilities so far has been synthesized by combining dithienyl-DPP and (E)-2-(2-(thiophen-2-yl)vinyl)thiophene moieties (P15b) [106] and [1,2-bis(5-(thiophen-2-yl)selenophen2-yl]ethene (P16) [17], which are intense conjugated n-type [11] and p-type moieties [83,94], respectively. A third strategy is to modify the original conjugation structure. Lei et al. modified PPV by coupling its repeating unit with amide and ester groups to form a larger conjugation system (P17), which was stabilized by multiple interaction such as with-drawing electron and hydrogen bonds [107]. Thus, inserting and/or copolymerization of intense conjugated units, and internal modifying of conjugation structure are thought to be promising strategies to develop materials with stronger conjugation and better electronic properties. 
Table 1. Summary of conjugated polymers indicating the effect of structures on the carrier mobility in TFT devices (the mobility is that of holes except P14 and P17).

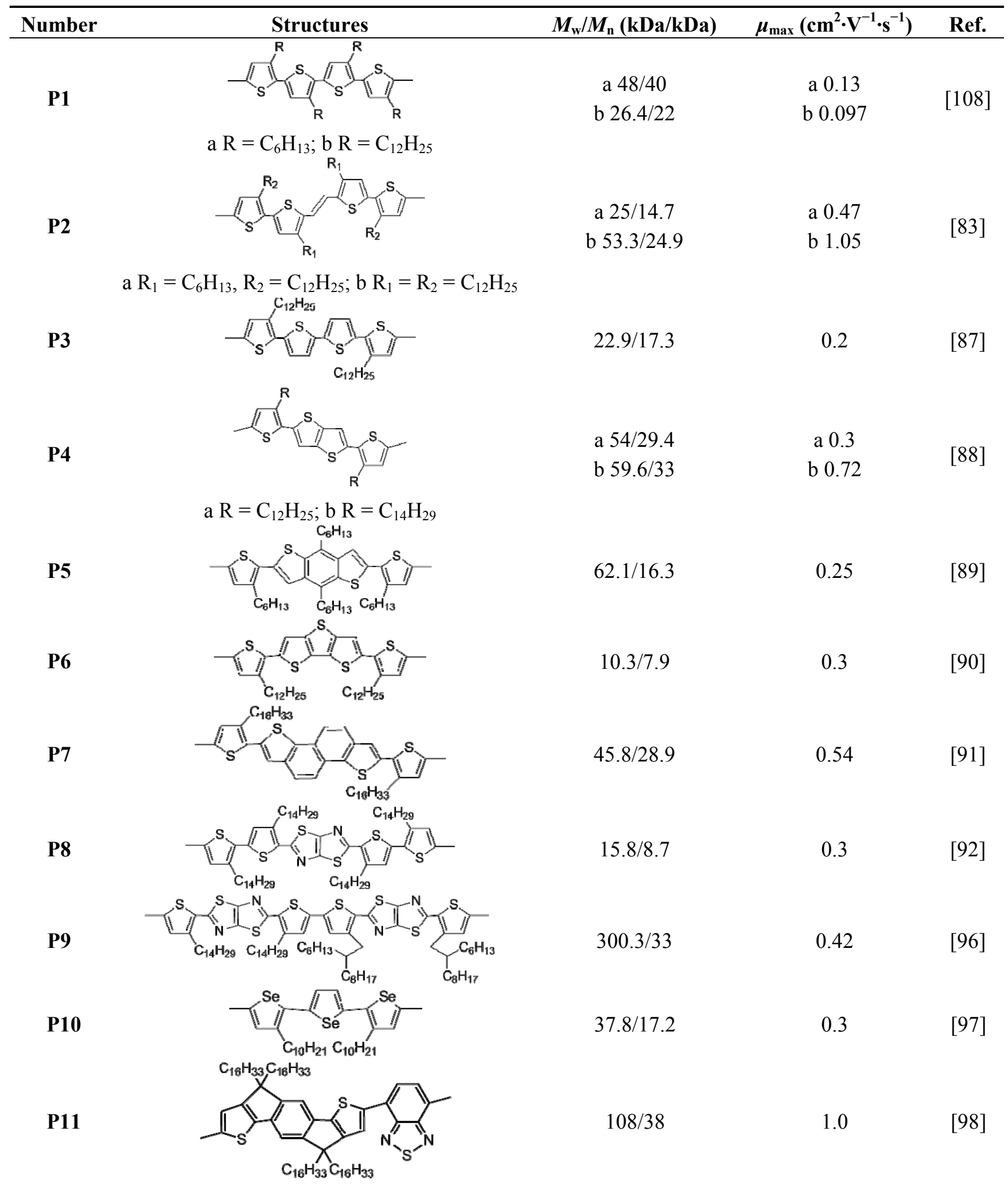


Table 1. Cont.

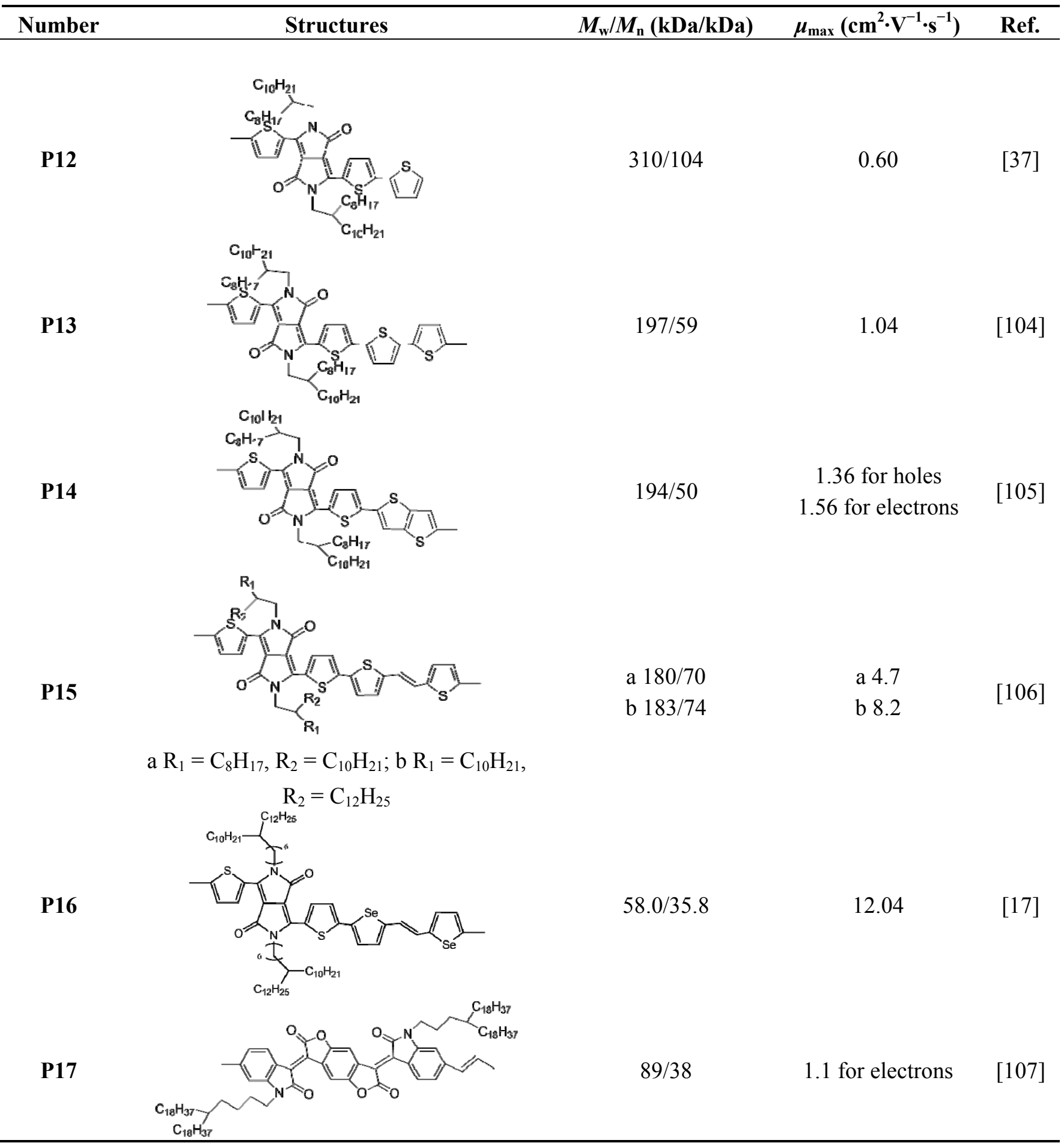

Besides the electronic effects, the different conjugated structures of backbones could lead to big changes of rigidity among the family of conjugated polymers, which influence the structures and morphology in turn. The rigidity of chains could be increased by a more intense conjugation $[68,79,109]$. Zhao et al. reported that the persistence length of pBTTT (P4) $(9.0 \mathrm{~nm})$ was much larger than rr-P3HT $(4.8 \mathrm{~nm})$ due to the introduction of fused thieno[3,2-b]thiophene moiety into backbones to strengthen the conjugation, implying a more rigid chains [68]. More rigid chains tend to adopt extended rod-like conformation, which is prone to aggregate orderly and form the precursor for domains with a large size 
and good performance in pBTTT films. Utilizing single molecule spectroscopy (SMS), Hu et al. also reported that with the gradually more intense conjugation in chains brought by a better planarity (for PQT12, P3), highly delocalized thienothiophene fused aromatic rings (for pBTTT) and a much higher planarity due to intra-chain donor-acceptor charge transfer (for pTzQT, P8) compared than P3HT, the content of single chains with an extended conformation in solutions increased, which suggested a more ordered and aligned rod-like film morphology arising from these rod-like single chains [109]. Thus, it is proven that the conformations of conjugated polymers display more anisotropic and extended characteristics as a result of planarity and rigidity $[68,69,109]$, which have an important effect on the self-assembly and film morphology in terms of the stacking order.

As mentioned in Section 2.1, an H-aggregation with enhanced order or an appearance of J-aggregation could be indicated by a red-shifted max absorption and/or an enhanced ratio of $A_{0-0} / A_{0-1}$ (this ratio $<1$ for $\mathrm{H}$-aggregation and $>1$ for J-aggregation) in UV-Vis absorption spectra, which is stable in electronic energy and favored for charge transport. The conjugated polymer chains coupling with more intense conjugated units [79,99,110] or electron-rich units [99] display a longer conjugation length and more extended conformation which could be reflected by the red-shift max absorption in solutions [68,109]. These cause a regular stacking of planar chains to form ordered crystallites as efficient charge transport pathways, indicated in red-shifted absorption spectra and arc-like patterns in 2D-WAXS of films [99,110-112]. Here, it is noted that the inter-chain interaction between donor units and acceptor units could lead to two stacking modes for donor-acceptor copolymers such as P(NDI2OD-T2) [113] and F8BT [114], that is, alternating column-like stacking of donors and acceptors respectively (Form I) and mixed stacking between donor and acceptor (Form II) (Figure 8). By thermal annealing, the Form I structure could transform into Form II, implying that Form II is the thermodynamic stable stacking mode. It might be attributed to the extensive intra-chain and inter-chain donor-acceptor interactions to lower the energy of the conjugation system and the minimized steric repulsion of side-chains in the Form II structure [113]. However, the kinetic stable Form I stacking was favored for its straight inter-chain hopping pathways provided by column-like stacking donors(acceptors) like the example of F8BT [114], which could be reflected also by its higher absorption at $810 \mathrm{~nm}$ indicating a more ordered and enhanced aggregation in the example of P(NDI2OD-T2) [113].

Besides stacking order of chains, intrinsic conjugation structure and morphological features could be also dominated by molecular structures in terms of textures in films, size of crystallites or crystalline domains, and anisotropy. Firstly, the textures of dispersed nanowires and parallel aligned nanofibrils are frequently observed in films. Because more planar and intensely conjugated chains tend to stack more orderly and achieve a higher crystallinity, more fibrillar morphology beneficial for charge transport arising from uniaxial $\pi-\pi$ stacking of extended chains will be formed accordingly compared with the featureless films of polymers with larger torsions [115], less intense conjugation [37,116] and/or conformation not favored [86] (Figure 9). Secondly, the bigger size of crystallites or crystalline domains could be acquired by increasing the conjugation degree of backbones. As to the domain size in liquid-crystal-like films, DPPT-TT (P14) with the stronger conjugation of backbone had a size of about $1.2 \mu \mathrm{m}$, larger than DPPT-2T (P13) with weaker conjugation and a size of about $500 \mathrm{~nm}$ as reported by Zhang et al. (Figure 10) [37]. The larger domain size is thought to originate from the fibrillar structure of a larger size as a result of more conjugated backbones. Thirdly, the anisotropy of films crucial for device performances could be controlled by the molecular structures of chains [117-119]. 
A larger dichroic ratio of optical absorption or emission could reflect the anisotropy of charge transport in films, which could be used to guide the design and choose proper electronic materials. The larger anisotropy reflected by a bigger dichroic ratio (4-5) in films with a thermodynamic more stable stacking structures than that (2-2.5) with a kinetically trapped stacking structure for P(NDI2OD-2T) was reported by Brinkmann et al. (Figure 8a-a') [113]. However, the examples of comparison of anisotropy in films between polymers with different backbone structures are few. Higashi et al. prepared aligned films of pBTTT and pBTCT using capillary action and post-annealing (Figure 11). The optical dichroic ration of pBTTT and pBTCT aligned films were 1.18 and 1.13 before annealing, and 1.56 and 1.17 after annealing, respectively [120]. The higher anisotropy in pBTTT aligned film may be due to its better stacking of chains arising from the more straight shape, extended conjugation of backbones and ordered stacking of side-chains like the examples by Lei et al. [86] and He et al. [121]. Therefore, the effect of backbone structure on anisotropy still needs to be understood better for applications in the future.

\subsection{Backbone Shape}

As a result of combination of backbones and side-chains, the shape of chains also affects the conformation a lot mainly in terms of curvature of backbones (Figure 12) [86,111,112,122]. Many groups of conjugated polymers based on moieties such as isomers of benzodithiophene, naphthodithiophene and dithienocarbazole, etc., have been synthesized to tune the backbone curvature. Rieger et al. simulated the conformation of backbones inserted by different isomers of benzodithiophene and found that the monomer angle, amplitude and wavelength of the wave-like conformation could be controlled by the configuration of benzodithiophene rings (Figure 12a) [122]. Although an increased degree of curvature improved the solubility, it decreased the conjugation order in the film. An intermediate degree of curvature yielded the most intense conjugation of chains. Osaka et al. obtained different conformation of backbones by inserting different isomers of naphthodithiophene rings, such as zigzag shape, square wave, pseudo straight and sine wave (Figure 12b) [112]. They found that the HOMO delocalization in pseudo straight and sine wave shape conformation was better than that in zigzag and square wave shape with sharp bending of rings. The pseudo straight conformation of chains was promising for the extension of intra-chain conjugation and better overlaps of inter-chain HOMO orbitals. Deng et al. provided a clearer image for the conformation of donor-acceptor copolymers based on dithienocarbazole [111]. With sulfur atoms in the same side in the dithienocarbazole moiety, the curvature of backbones was huge compared to the wave-like conformation of copolymer with sulfur atoms in the different sides (Figure 12c). Lei et al. proposed a general guideline for optimizing the conformation of chains as inserting centrosymmetric donor moieties into the backbones (Figure 12d) [86]. By this method, the backbone displayed a straight planar conformation instead of the wave-like conformation for backbones containing axial symmetric moieties. Similar to the best conjugation of chains and electronic properties of pseudo-straight shape chains reported by Osaka et al. [112], the straight planar conformation led to a better carrier mobility than wave-like ones in donor-acceptor copolymers. Thus, a straight or moderate wave-like backbone shape is thought to lead to a conformational mode beneficial for an enhanced conjugation of chains. 
Figure 8. The HR-TEM photos and corresponding fast Fourier transforms of Form I and Form II stacking structures in (a); the schematic illustration of Form I and Form II in (a') and the polarized UV-Vis spectra of Form I (upper) and Form II (down) in (a") for P(NDI2OD-2T) (Adapted with permission from [113]. Copyright 2012 American Chemical Society); The variation of Raman intensity ratio representing inter-chain torsion with molecular weight (larger ratio for larger torsion) in (b); the schematic illustration of conformational change due to different inter-ring torsion in (b') and the UV-Vis spectra in (b') for F8BT, (Adapted with permission from [114]. Copyright 2005 American Chemical Society).
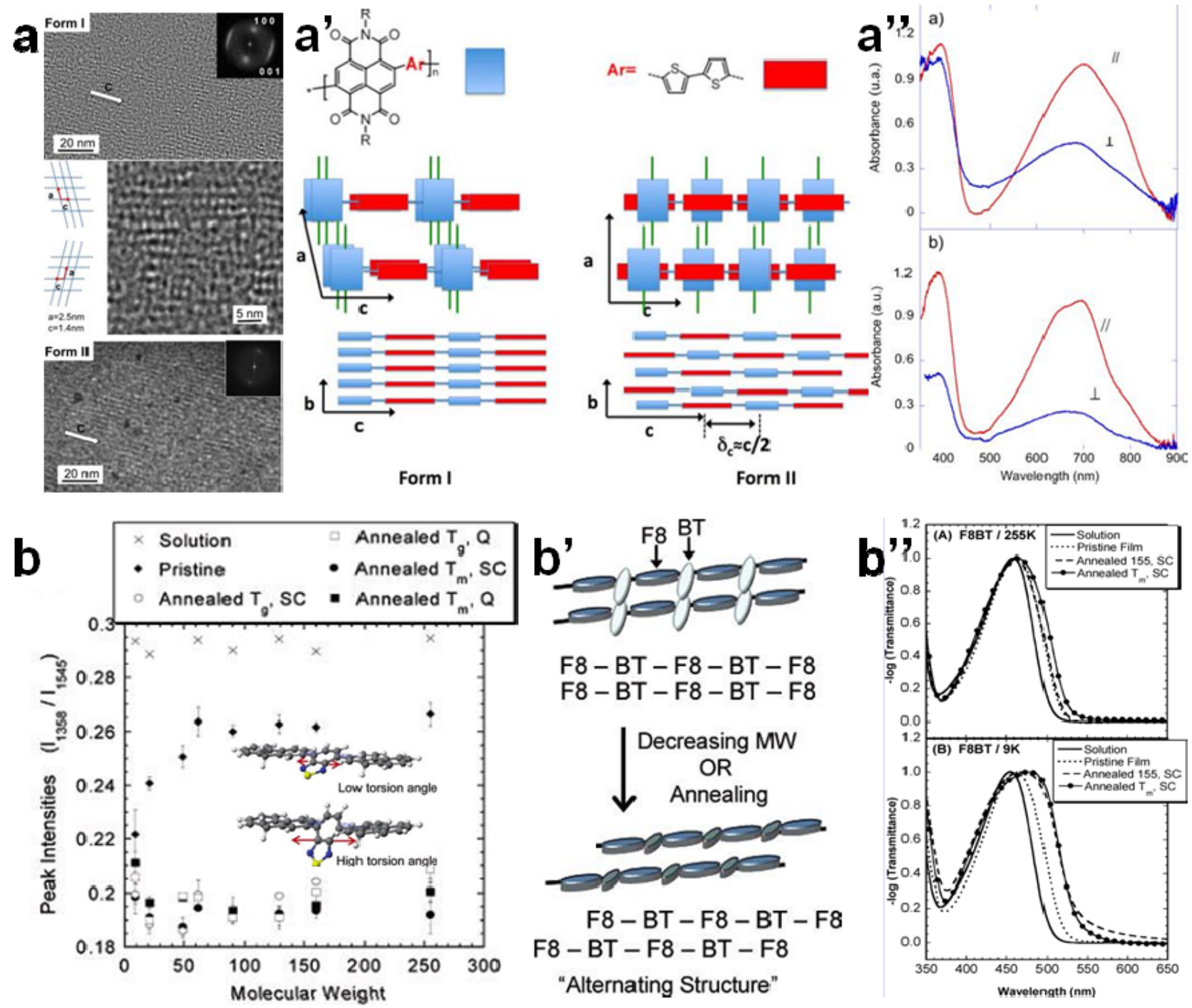

$\mathrm{F} 8-\mathrm{BT}-\mathrm{F} 8-\mathrm{BT}-\mathrm{F} 8$
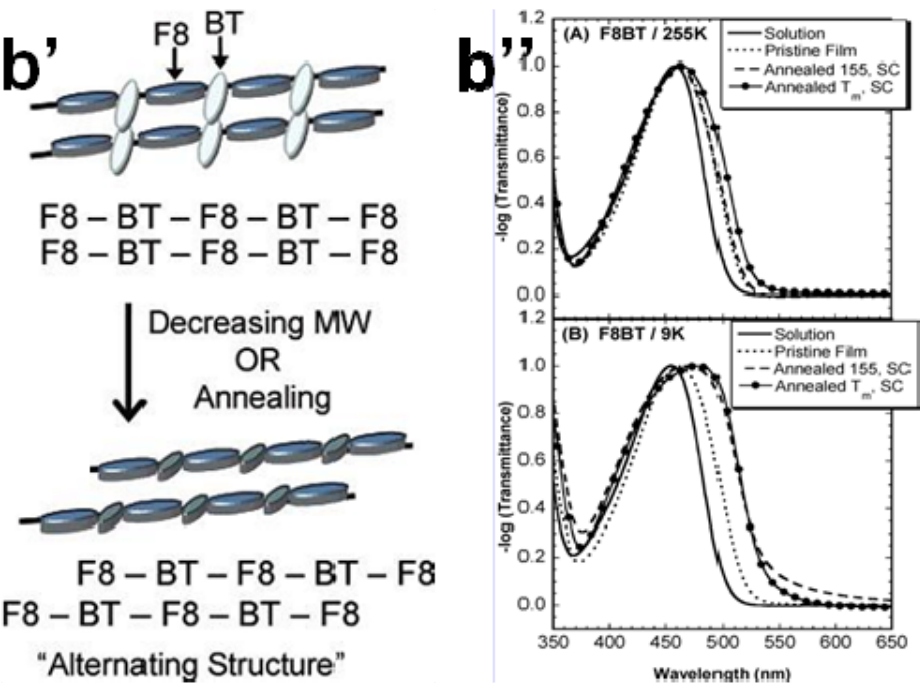

"Alternating Structure"

$\mathrm{F} 8-\mathrm{BT}-\mathrm{F} 8-\mathrm{BT}-\mathrm{F} 8$

$\mathrm{F} 8-\mathrm{BT}-\mathrm{F} 8-\mathrm{BT}-\mathrm{F} 8$ 
Figure 9. (I) Atomic force microscopy height images for (a) as-spun films of PDTST; (b) as-spun films of PDTQT; (c) films of PDTST annealed at $100{ }^{\circ} \mathrm{C}$; and (d) films of PDTQT annealed at $100{ }^{\circ} \mathrm{C}$ (Reprinted with permission from [115]. Copyright 2010 American Chemical Society); (II) AFM topography images $(2.0 \mu \mathrm{m} \times 2.0 \mu \mathrm{m})$ of spin-cast and $200{ }^{\circ} \mathrm{C}$ annealed films of (a) BTT-T and (b) BTT-TT and polarized optical microscopy (POM) images (50× magnification, reflection mode) for (c) BTT-T and (d) BTT-TT (Reprinted with permission from [116]. Copyright 2011 American Chemical Society); (III) AFM height images of DPPT polymer films. (a) DPPT-TT, as-cast; (b) DPPT-2T, as-cast; (c) DPPT-TT, annealed; (d) DPPT-2T, annealed; All scale bars denote $200 \mathrm{~nm}$ (Adapted with permission from [37]. Copyright 2011 American Chemical Society); and (IV) AFM height images of (a) IIDDT, (b) IID-BDT, (c) IIDT, and (d) IID-TTT films spin-casted from TCE solution $(4 \mathrm{mg} / \mathrm{mL})$ on OTS-treated $\mathrm{SiO} 2 / \mathrm{Si}$ substrate and annealed at $150{ }^{\circ} \mathrm{C}$ for $30 \mathrm{~min}$ (Reprinted with permission from [86]. Copyright 2012 American Chemical Society).
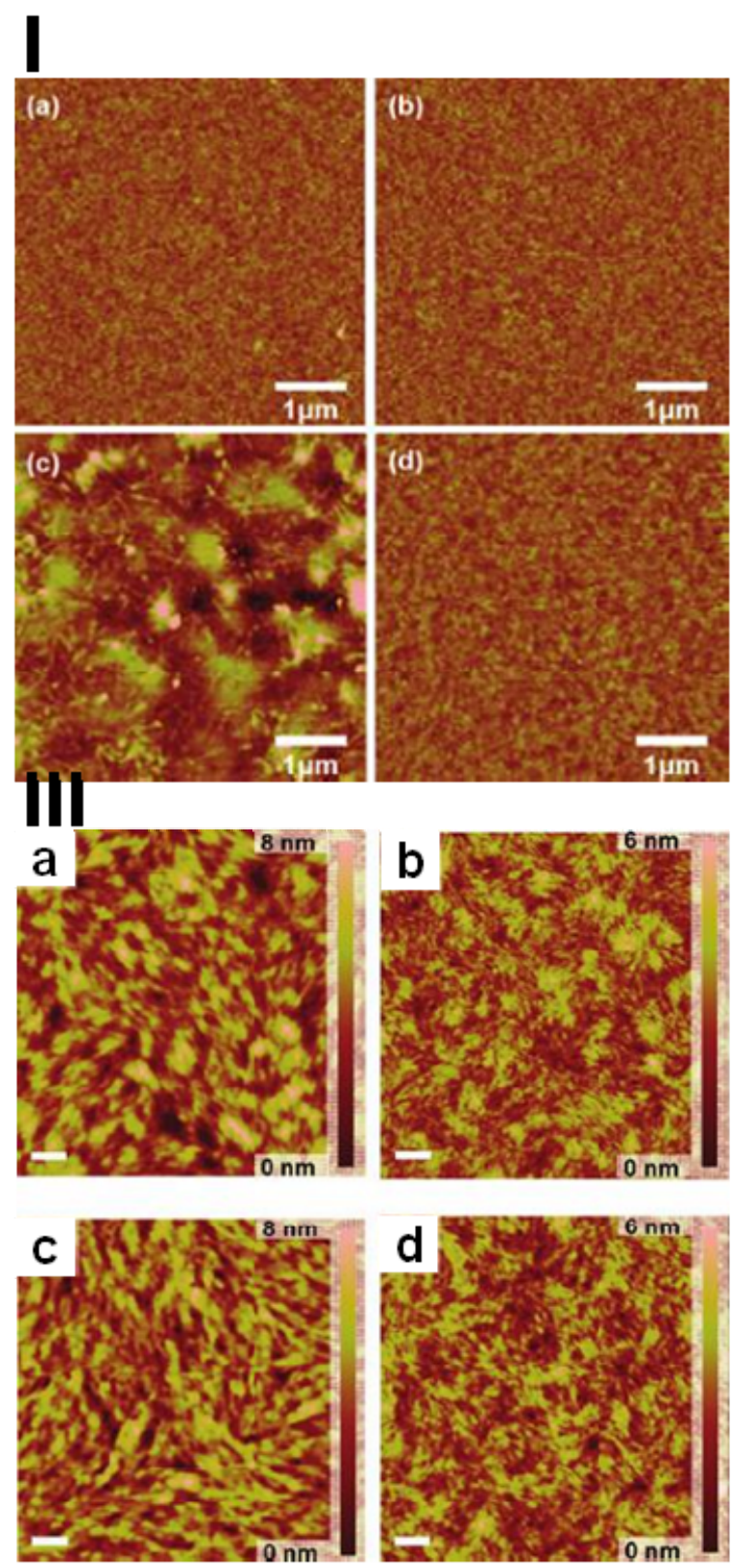
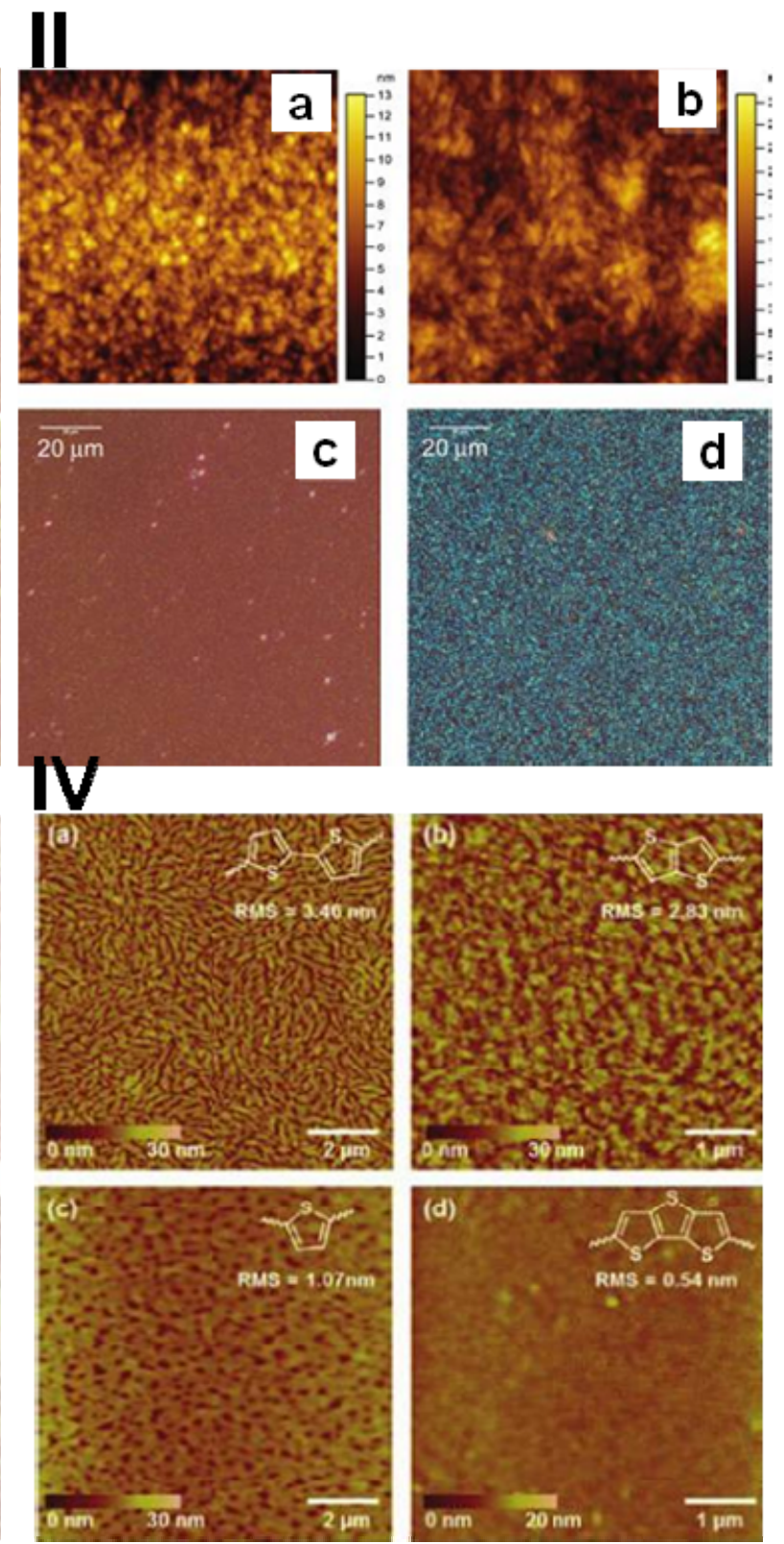
Figure 10. Orientation maps of (a) DPPT-TT and (b) DPPT-2T obtained according to dark-field TEM images, which indicate the domain size with scale bars representing $2 \mu \mathrm{m}$, (Adapted with permission from [37]. Copyright 2011 American Chemical Society).
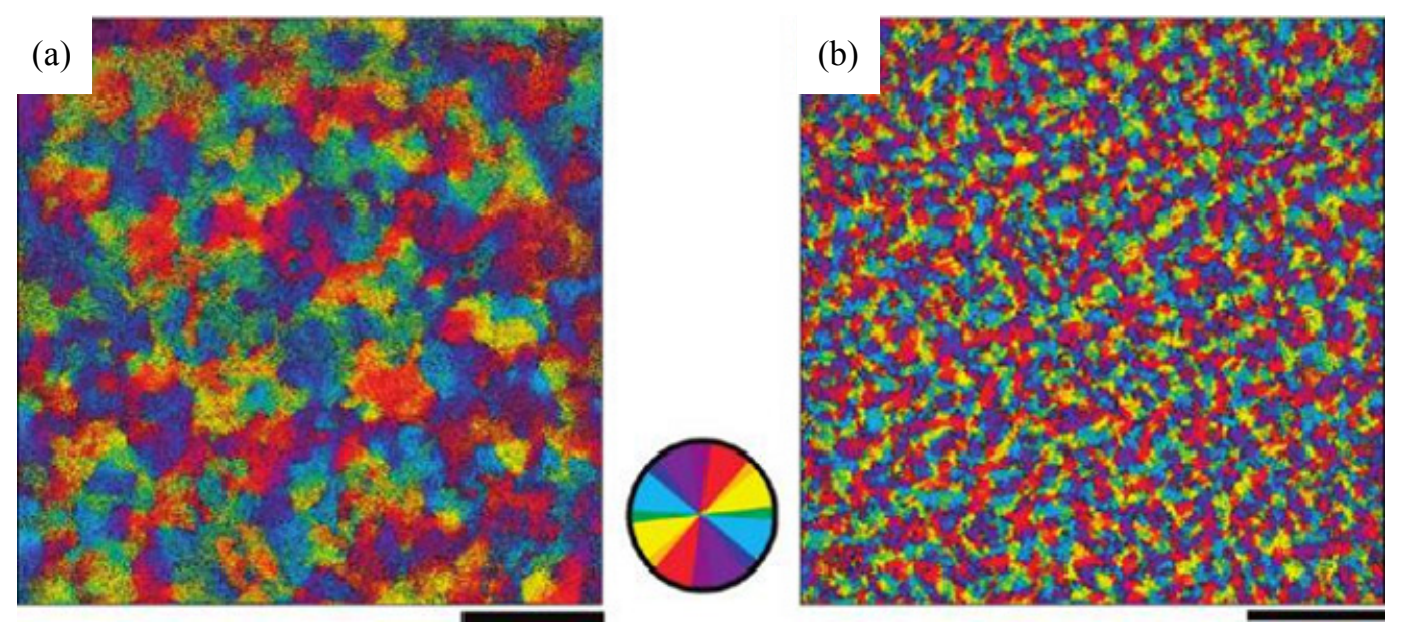

Figure 11. Schematic diagram of polymer film fabrication using capillary action in (a) and micrographs of the pBTTT (upper) and PBTCT films in cross-polarized light with their molecular structure, the arrows denoting the solution flow direction in (b) (Adapted with permission from [120], Copyright Applied Physics Express 2011 The Japan Society of Applied Physics).

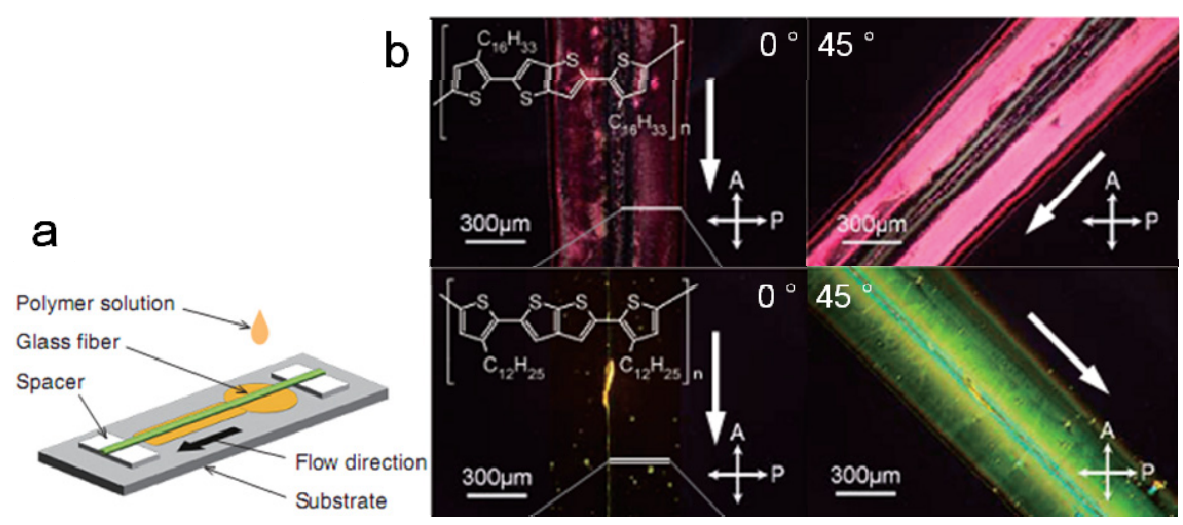

\subsection{Side-Chains}

Apart from the influence of backbones, distribution of side-chains can not be ignored. The density, length, volume and branching of side-chains play important roles in the planarity and conjugation intensity of chains. Firstly, the side-chain motions occur before backbone motions and they could drive the conformational change of backbones [123]. The longer and/or branched alkyl side-chains and larger alkyl side-chain density could cause an increase of the inter-ring torsion and lead to a relatively more coil-like and flexible conformation, which could tune aggregation and transport properties in turn (Figure 13a). Firstly, Oosterbaan et al proposed that the morphology at FET active interface could be tuned by side-chain length for P3ATs [124]. The solubility of P3AT with a shorter side-chain was larger and it started to aggregate at early stage during casting, resulting in a longer time for crystallites growth. As a result, the roughness of films for P3AT with shorter side-chains and more intense 
conjugation was larger indicating a larger crystallinity, which was the origin of the highest mobility in its film. Besides, a larger dichroic ratio of optical absorption or emission indicating the higher anisotropy of carrier transport could be tuned by the length of side-chains [117,119]. Ren et al. reported that with shorter side-chains, the dichroic ratio of films prepared by mechanically shearing turned larger [117]. They attributed it to the higher $T_{\mathrm{g}}$ of PPV with shorter side-chains to obtain orientation due to less relaxing of chains. In fact, the stronger conjugation in backbone plane determines a larger anisotropy of itself, which leads to larger anisotropy in films. Bologenesi et al concluded the structural features to obtain larger anisotropy for polythiophenes as (1) high regio-regularity; (2) more substituted thiophene rings in the main chain; and (3) the higher the ratio of molecular weight/length of alkyl side-chain. In application of commercial P3ATs, chains with shorter chains and larger molecular weight may have a larger anisotropic in films. Secondly, the volume of side-chains, i.e., alkyl side-chains or conjugated side-chains could increase the inter-ring torsion and decrease the degree of intra- and inter-chain conjugation (Figure 13b) [86,115]. A excess torsion could lead to a less ordered morphology and hamper the efficient transport of carriers, as reported by Park et al for a polythiophene with a larger density of thiophene side-chain and a lower mobility of $5.1 \times 10^{-2} \mathrm{~cm}^{2} \cdot \mathrm{V}^{-1} \cdot \mathrm{s}^{-1}$ than that with a lower density and $3.0 \times 10^{-3} \mathrm{~cm}^{2} \cdot \mathrm{V}^{-1} \cdot \mathrm{s}^{-1}$ (Figure 9I) [115]. Besides, the steric effect arising from the head-head linkage of alkyl thiophenes in backbones results in a huge torsion (Figure 13c), which destroys the planarity and conjugation of chains and leads to a bad carrier mobility [50]. Therefore, a high regio-regularity (fewer head-head linkages) is wanted for polythiophenes and their derivatives.

Secondly, the looser distribution of side-chains could relieve the impact of increasing torsion and/or steric effect of side-chains and improve the planarity and conjugation in chains, promoting more ordered self-assembly [86,115]. Meanwhile, the ordered stacking of side-chains could induce and improve the crystallization of backbones in turn. For P3ATs, it is found that along with increasing the solubility of polymers the longer side-chains could pack orderly with the conformations close to all-trans like in crystalline lipids. This crystallization-like packing of side-chains is thought to induce the ordered packing of backbones to form more ordered crystallites [125,126]. Meanwhile, the ordered tilted-interdigitation stacking of long alkyl side-chains could also promote the better stacking of backbones in turn and improve the device performance [54,127,128]. Sauve et al. also reported that for thermal annealed P3DDT films, the out-of-plane XRD profile of it showed higher peaks of (400) and (500) diffractions than pristine films [108]. The more ordered crystalline structure arising from more regular side-chain stacking led to the largely enhanced mobility for annealed films $\left(0.097 \mathrm{~cm}^{2} \cdot \mathrm{V}^{-1} \cdot \mathrm{s}^{-1}\right)$ than pristine films $\left(0.004 \mathrm{~cm}^{2} \cdot \mathrm{V}^{-1} \cdot \mathrm{s}^{-1}\right)$. Alternatively, the strategy of decreasing the side-chain density is preferred to promote the ordered packing of side-chains via tilting and interdigitation to reach the packing density in crystalline $\mathrm{PE}$, which is beneficial to improve the crystalline order and charge transport in the examples of PQT and pBTTT (Figure 13d) [82,129-131]. Wang et al. reported that the higher carrier mobility of pBTTT films $\left(0.34 \mathrm{~cm}^{2} \mathrm{~V}^{-1} \mathrm{~s}^{-1}\right)$ than P3HT $\left(0.10 \mathrm{~cm}^{2} \mathrm{~V}^{-1} \mathrm{~s}^{-1}\right)$ originated from its higher intrinsic carrier mobility of crystallites $\left(20 \mathrm{~cm}^{2} \mathrm{~V}^{-1} \mathrm{~s}^{-1}\right.$ for pBTTT and $0.83-1.57 \mathrm{~cm}^{2} \mathrm{~V}^{-1} \mathrm{~s}^{-1}$ for P3HT), considering their similar crystalline size of about $10 \mathrm{~nm}$ [130]. They attributed it to the 3-D ordered crystallization of pBTTT due to its strong backbone rigidity and interdigitation of side-chains. Instead, a less ordered stacking of side-chains arising from zigzag backbones (Figure 9IV) [86] or substitution of side-chains on the same side of fused aromatic rings [121] (Figure 13d) increased the lamellar period and destroyed the ordered stacking of lamella. Recently, introducing longer side-chains 
with branching point further from backbones has been found to promote a stronger interchain $\pi-\pi$ stacking indicated by the decreased $d_{\pi-\pi}$, for the steric effect of bulky branching side-chains on the aggregation of adjacent backbones has been relieved [17,132]. Accordingly, the carrier mobility has been largely enhanced due to the smaller $d_{\pi-\pi}$ [17,132], like P-29-DPPDTSE $\left(\right.$ P16, $d_{\pi-\pi}=3.58 \AA$, $\left.\mu_{\max }=12.04 \mathrm{~cm}^{2} \cdot \mathrm{V}^{-1} \cdot \mathrm{s}^{-1}\right)$ and P-24-DPPDTSE $\left(d_{\pi-\pi}>3.7 \AA, \mu_{\max }=4.4 \mathrm{~cm}^{2} \cdot \mathrm{V}^{-1} \cdot \mathrm{s}^{-1}\right)$ [17]. Especially for some existing groups of high-mobility conjugated polymers, this strategy about side-chains is very promising to reach an extremely high device mobility.

Figure 12. (a) Geometry of the polymers: the monomer angle refers to the angle which is introduced by the corresponding benzodithiophene into a linear polymer chain (Reprinted with permission from [122]. Copyright 2010 American Chemical Society); (b) Optimized backbone structures of the polymers based on isomers of naphthodithiophene (Reprinted with permission from [112]. Copyright 2011 American Chemical Society); (c) Optimized backbone conformation of the polymers base on isomers of dithienocarbazole (Reprinted with permission from [111]. Copyright 2012 American Chemical Society); and (d) The cartoon representation of copolymers with different symmetries and their film packing (Adapted with permission from [86]. Copyright 2012 American Chemical Society).

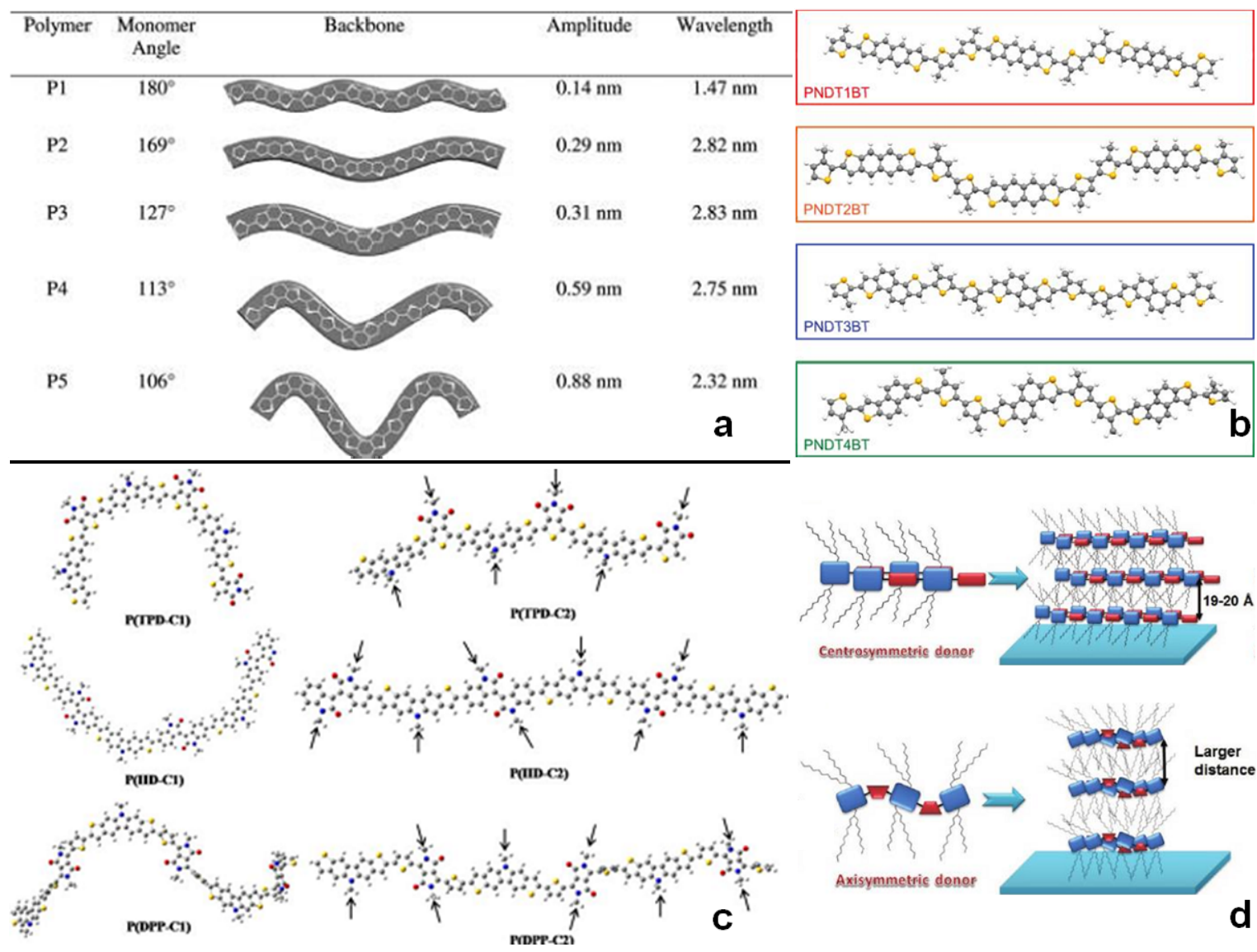


Figure 13. (a-c) Schematic illustration of side-chains on the inter-ring torsion in backbones; (d) the calculated side-chain packing density with varying tilting angle and the optimal stacking mode for P3HT, PQT and pBTTT, (Adapted with permission from [129]. Copyright 2007 American Chemical Society) and (e) the illustration for effect of side chain substitution position on the lamellar stacking mode, (Adapted with permission from [121]. Copyright 2009 American Chemical Society).
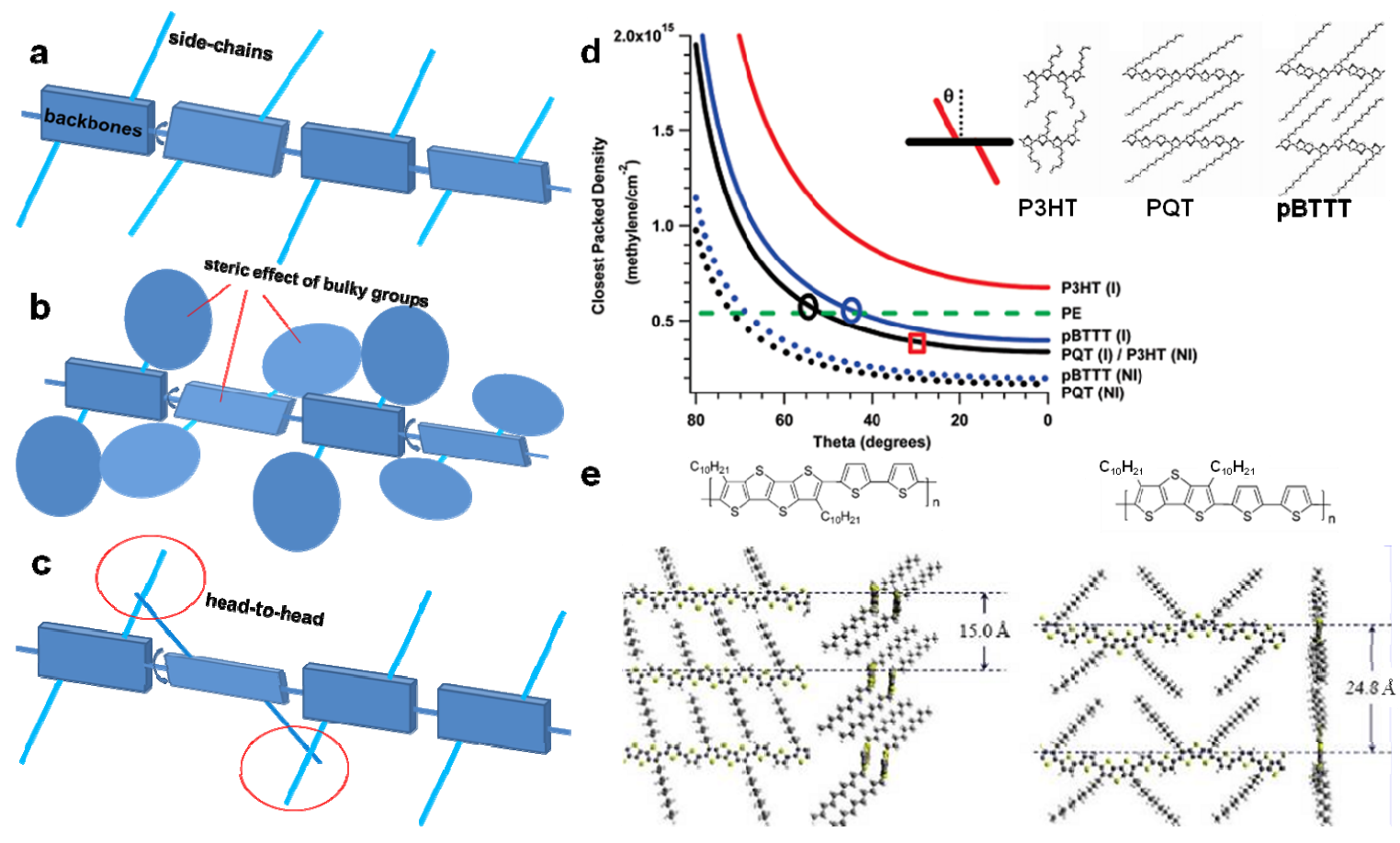

\subsection{Molecular Weight}

As mentioned above, the molecular weight could control the size of crystals depending on the inter-ring torsion barrier of backbones (Figure 6II, A). Besides, molecular weight exerts effects on the crystallization morphology of conjugated polymers by controlling the connectivity between crystalline regions. As to the lamellar structure [71], the total lamellar thickness increased with the size of crystalline region nearly unchanged with the increasing molecular weight. The bending of chains near the ends gave rise to disorder region in lamella and tie chains connecting adjacent lamella were observed in thickened disorder region by HR-TEM for high molecular weight P3HT (Figure 6I). Kline et al. reported that for $\mathrm{P} 3 \mathrm{HT}$ with a higher molecular weight the transport barrier was smaller (54 meV) than that of P3HT with lower molecular weight (>60 meV) [44]. They attributed it to that the long chains could interconnect ordered areas and prevent charge carriers from being trapped by the disordered boundary regions. Accordingly, the spin-coated films of P3HT with a high molecular weight displayed better mobility than that of lower molecular weights. For many conjugated polymers, the chains with lower MWs could form regular crystals with large sizes but lack sufficient connections with each other. For chains with larger MWs, though granular morphology is often formed with much smaller-sized crystalline regions, the connections between adjacent crystallites are improved (Figure 14) [44,133]. Besides, the $\pi-\pi$ interactions between conjugated units is enhanced due to more segments, leading to stronger intra-chain and inter-chain conjugation indicated by red-shifted spectra 
and increased $0-0$ absorptions in films. The trade-off between the size of crystalline regions with high carrier mobilities and their connections by tie-chains dominates the optimal MW for the highest carrier mobility [96,133]. Besides electronic properties, the chains with larger MWs have problems like larger units hard to diffuse, lower solubility and severe entanglements, which limit the ordered aggregation of them and need solutions being discussed in Section 4.

Figure 14. Model for transport in (a) low-MW and (b) high-MW films. Charge carriers are trapped on nanorods (highlighted in yellow) in the low MW case. Long chains in high-MW films bridge the ordered regions and soften the boundaries (marked with arrow) (Adapted with the permission from [44]. Copyright 2005 American Chemical Society).

(a)
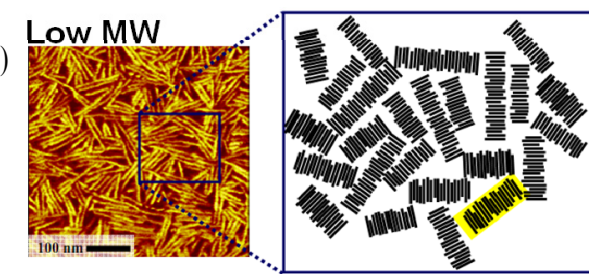

(b)

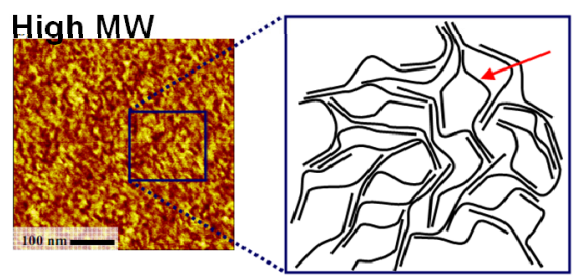

In this section, we discuss the influences of intrinsic molecular structures on the structures, morphology and charge transport of conjugated polymers. It is proposed that intrinsic molecular structural features such as (1) extension of conjugation system and strengthening intra-chain and inter-chain interaction; (2) shapes of backbones; (3) distribution of side-chains; and (4) molecular weight are crucial factors to tailor the conjugated pathways for charge transport and control the conformation for ordered aggregation (Figure 15). Although the design of molecular structures is crucial for development of next generation high-performance materials, the art in preparation of devices from solution is also important to realize the potential of these solution-processable polymer materials fully for industry applications. Therefore, the behavior of conjugated polymers in solution and the processing strategies for films from solution will be discussed in following.

\section{The Optimization of Solution State of Conjugated Polymers to Promote Order in Films}

One of the out-standing advantages of conjugated polymers in (opto-)electronic applications is solution-processing, which is cheaper and more simple than chemical vapor deposition (CVD) of conjugated small molecules and adapts well with industry manufacturing, i.e., roll-to-roll printing. However, the intrinsic intra-chain conjugation and inter-chain interactions of conjugated chains different from flexible chains give rise to diverse aggregation characteristics and self-assembly behavior. Besides, the dissolution and aggregation of chains depend also on the parameters of solutions, i.e., temperature, concentration, solubility parameters, polarity, specific interaction between solvent and solute, etc. All these factors increase the complexity of controlling solution state of chains for the final film morphology and performances. Therefore, the features of solution state for conjugated polymers, their 
influences on the film morphology and performances, and the methods of optimizing solution state to prepare better functional films will be discussed in following.

Figure 15. Schematic illustration of the structural factors influencing the aggregation of charge transport property of conjugated polymers.

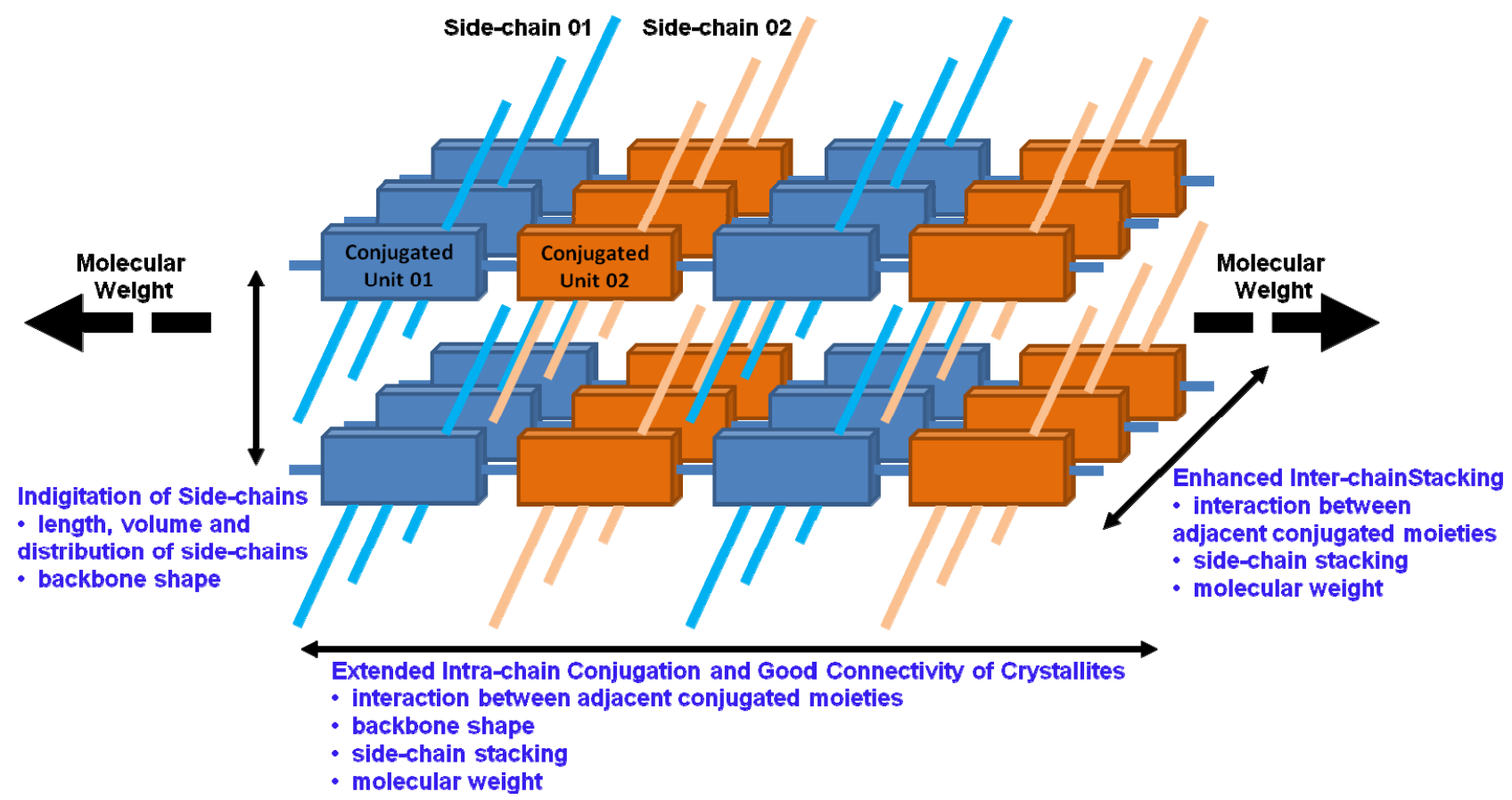

\subsection{The Influences of Solution State on the Morphology and Performance of Conjugated Polymer Films}

\subsubsection{Equilibrium between Coil Unimers and Disordered Aggregates}

In a good solution of polymers, the chains are divided by a large amount of solvent molecules and therefore most chains are far away from each other. In other words, they are free chains. Meanwhile, the conformation of them is coil-like. However, due to the elongated persistence length of chains arising from conjugation plane in backbones, the single chains of conjugated polymers display a more anisotropic extended conformation with less bending like paperclips, compared to the isotropic and disordered coils of more flexible chains (Figure 5a) [69]. Besides, the chain-folding in backbones for conjugated polymers is a more extended one than flexible chains, i.e., seven thiophene rings in the folding section in P3HT and nine for P3DDT (Figure 5b) [70]. The rigid folding results in a severe entanglement of conjugated polymer chains according to the theory reported by Wu et al. [134]. They modeled the entanglement of polymer chains as a binary hooking contact of Kuhn nodes between two chains, and the number of real or virtual skeletal bonds in an entanglement strand was equal to $3\left(C_{\infty}\right)^{\alpha}$, where $C_{\infty}$ and $\alpha$ were the characteristic ratio of chains and the number of hooks involved at an entanglement junction, respectively (Figure 16a). As known, the characteristic ratio of polymer chains increases with their increased rigidity. Therefore, for conjugated polymers with a stronger planarity and rigidity, the rigid nature of folding and more bonds in the entanglement strand lead to more severe and firm entanglements of chains arising from more extended conformations prevailing [109] indicated 
by a bigger $C_{\infty}$. As proven by Wen et al. [135] and Huang et al. [136,137], there were disordered spherical aggregate clusters made of chains entangled according to the results of dynamic light scattering (DLS) and static light scattering (SLS) even in a good solution, i.e., poly[2-methoxy-5-(2-ethylhexyloxy)1,4-phenylenevinylene] (MEH-PPV) solutions in chloroform. The larger the size of aggregates is, the more chains are entangled within them. Meanwhile, the interior dynamics of the aggregate clusters revealed that segmental motions within are generally more suppressed than for non-aggregated polymer chains in solutions. Due to the disordered entanglements of chains, they are prevented from reorganization into ordered aggregates. Fortunately, Huang et al. [136,137] revealed that equilibrium between single coil chains and disordered spherical aggregates existed in solution. According to results of DLS, the single chains had a hydrodynamic radius around $4 \mathrm{~nm}$ and the spherical aggregates around $90 \mathrm{~nm}$. By decreasing the temperature of casting solution, the content of aggregates in solution decreased and that of single coil chains increased, leading to more fibrils appearing in the corresponding film. According to this result, it was thought that the single coil chains in solution is the origin of ordered aggregates such as nanofibrils in films (Figure 16b) [136]. Therefore, it is important to disentangle the single chains in disordered aggregates and increase the content of single coil chains in solution for their reorganization into ordered aggregates in a more stable way in energy. The methods for disentanglement of chains will be discussed later.

Figure 16. (a) The entanglement model reported by $\mathrm{Wu}$ : top are the schematic illustration of a real chain and its equivalent Kuhn chain and down are the types of inter-chain contacts associated with entanglement, (Adapted with permission from [134]. Copyright 1989 Wiley-VCH Verlag); and (b) The schematic illustration of equilibrium between unimer coils and associates in solution on the final film morphology and the corresponding TEM image of films. (Adapted with permission from [136]. Copyright 2010 American Chemical Society).
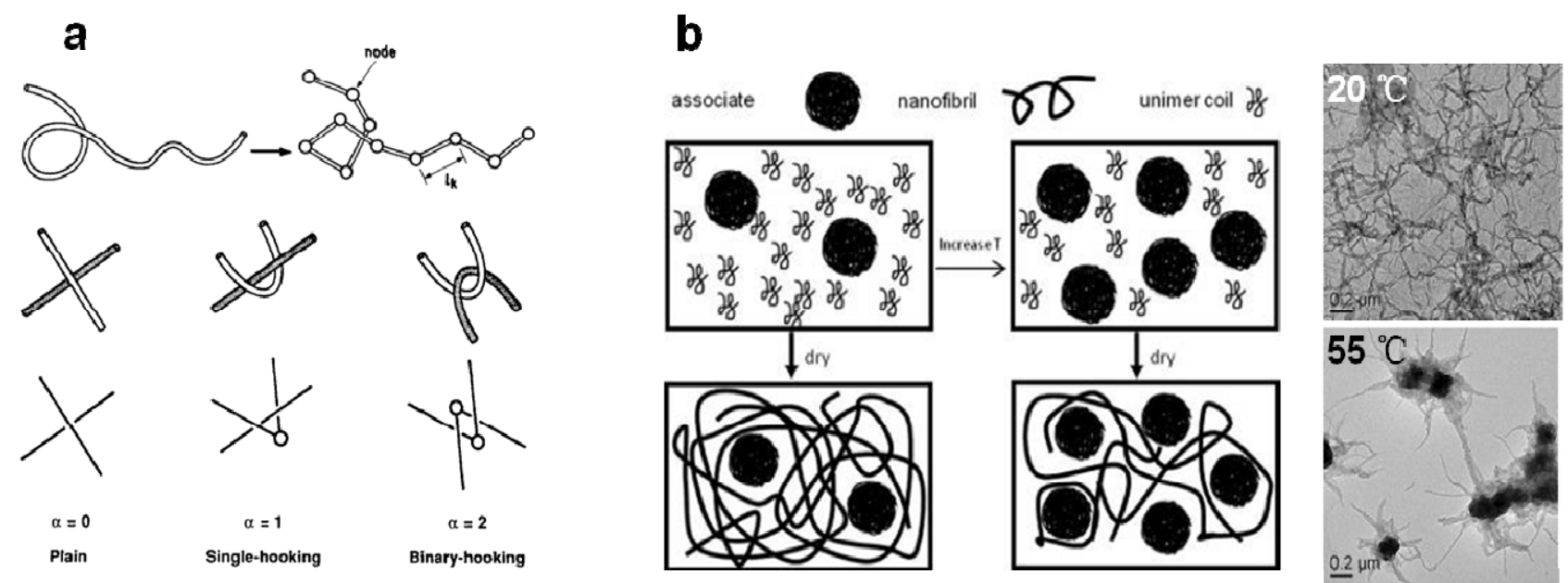

\subsubsection{The Conformation Transition and Ordered Aggregation in Solution}

Apart from the coil conformation more extended than flexible chains, the rod-like conformation is also crucial for the self-assembly of chains. Ruphooputh et al. reported the coexistence of coil-like and rod-like conformations in P3HT solutions and the coil-to-rod conformational transition via cooling the 
solution as early as in 1987, which was reflected by red-shift of max absorption indicating the electronic $\pi-\pi^{*}$ transition of chains. This red-shift of spectra implied the elongated conjugation length in chains. Besides, the coil-to-rod (or rod-to-coil) transition of chains conformed to an intra-chain mechanism because of the independence of this red-shift on concentration of solution [138]. Compared with "expanded" coil-like conformations, the anisotropy of chains in "collapsed" rod-like conformations is enhanced [29,139] due to the further elongated conjugation length and segment size driven by the intrinsic tendency for minimizing energy (Figure 17). Then, the enhanced intra-chain conjugation length strengthens the interchain $\pi-\pi$ stacking interactions of chains, which conforms to an inter-chain mechanism. During the aggregation of chains, the inter-chain stacking must happen after the intra-chain conformational transition due to the independence of chromism features on concentrations [138]. In other words, the coil-to-rod conformational transition play a decisive role in the ordered aggregation of chains, for the rod-like chains are the building blocks for ordered aggregates as transport pathways $[68,140,141]$. Meanwhile, the inter-chain stacking is closely related to the growth of ordered aggregates and cannot be ignored, especially for some donor-acceptor copolymers with a more-rod like conformation spontaneously in solution which need deeper research [128].

Figure 17. The schematic illustrations of equilibrium between coil-like and rod-like conformations in a (partly) dissolution state and the rod-like conformations in drying films after the coil-to-rod transition. The experimental histograms of modulation depth, $\mathrm{M}$ (larger M larger anisotropy), from single poly[2-methoxy-5-(2-ethylhexyloxy)-1,4phenylenevinylene] (MEH-PPV) molecules embedded in a PMMA matrix with different preparation methods: Spin-coated from (a) toluene and (b) chloroform solution with a considerable retention of coil-like conformations in solution; (c,d) The film samples were additionally solvent vapor annealed for $60 \mathrm{~min}$ with toluene-saturated atmosphere with rod-like conformations prevailing. (Adapted with permission from [139]. Copyright 2011 Wiley-VCH Verlag).

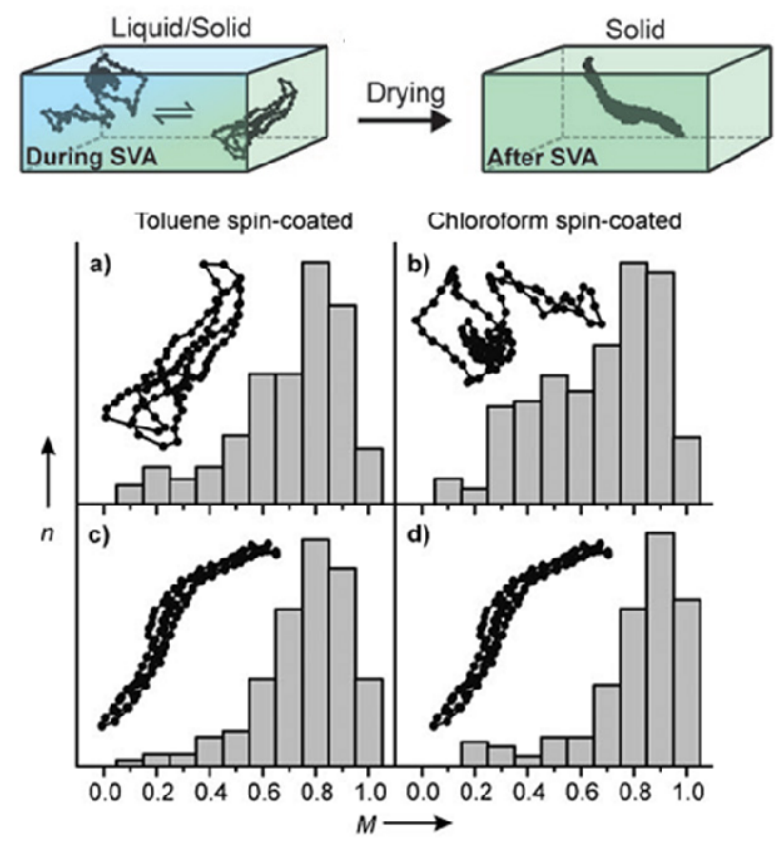




\subsection{The Strategy to Optimize the Solution State of Conjugated Polymers}

According to the discussions above, the optimization of solution state of chains should experience two stages sequentially as (1) disentanglement and (2) promotion of conformational transition towards rod-like and the rod-rod stacking followed. Firstly, disentanglement of single chains from disordered aggregates increase the content of free single chains for a further conformational transition into rod-like. Secondly, both the coil-to-rod transition and the following rod-rod stacking need a driven force to overcome kinetic barriers [142]. In following, the methods to solve the two problems above will be listed.

\subsubsection{Disentanglement}

The disentanglement of single chains from disordered aggregates means a relative motion of segments at the entanglement knot until both ends of one chain move out of this knot. During this process, the more bonds entangled, the large segment size and molecular weight, the interfering of side-chains and local $\pi-\pi$ interactions for conjugated polymers are adverse to the effective motion of segments out of knots. In terms of thermodynamic, one strategy is providing external energy to the system to overcome the disfavored decrease of entropy arising from entanglements. Huang et al. [137] and $\mathrm{Xu}$ et al. [143] reported that enhancing the temperature of solutions could increase the content of free single coil chains, which led to a more fibrillar morphology in the corresponding films. Thus, the importance of heating solutions before the next processing step [68,144,145] for the promotion of single coil chains is proved. Apart from thermal energy, ultrasonic vibration is also a effective method for disentanglement as reported by Zhao et al. (Figure 18I) [146,147]. They reported that the P3HT chains with a larger molecular weight had a more severe entanglement due to the binary-hooking contact prevailing arising from the folding and distortion of conformation of high- $\mathrm{M}_{\mathrm{W}}$ polymer. For high- $M_{\mathrm{W}}$ P3HT, the size of aggregates decreased and the amount of single chains increased after sonication, corresponding to the visually faster diffusion of solutes. The $0-0$ and $0-1$ absorbance in solution increased with the longer sonication time and reached a maximum for 6 min, also reflecting an assisted ordered $\pi-\pi$ stacking of single chains due to disentanglements. Accordingly, the amount of fibrils and roughness in films increased after sonication compared with the film cast from solution without sonication. Accompanied by the tendency of absorbance, crystallinity and anisotropy of films turning larger and saturated with longer sonication time, the carrier mobility also gradually increased and saturated for a time longer than $4 \mathrm{~min}$.

In terms of entropy, adding additives is another effective method for disentanglements. Wang et al. proposed the conformational shrinkage of originally extended and entangled MEH-PPV single chains after adding a small content $(<30 \%)$ of non-solvent nonane into the solution, which was reflected by the remarkably decreased solution viscosity (Figure 18II) [148], which led to a increase of entropy favored for the minimizing free energy of the system accompanied by the disentanglement of chains. Liu et al. also reported that the addition of n-dodecylthiol (12-thiol) into the solution of P3HT could remarkably decrease the size of aggregates and enhance the amount of single chains [149]. The more single chains led to more fibrillar morphology in P3HT/PCBM blend films beneficial for both optimizing phase separation and charge transport. It is found that the addition of $2.5 \mathrm{vol} \% 12$-thiol could obtain the best power conversion efficiency (PCE) of 2.61\% among the devices prepared from solutions with 
different contents of 12-thiol. Wang et al. reported that the effects of additive properties on the disentanglement of chains and the corresponding film morphology [150]. After adding a series of additives such as alkane, alkylthiols and alkyldithiols into pBTTT solutions in o-dichlorobenzene respectively, the original borderline solvent turned into a slightly bad solvent for chains, which led to a shrinkage of chain conformation both in aggregates and single chains reflected by DLS results. Accordingly, the segment motion driven by this shrinkage in the slightly bad solvent led to disentanglement, reflected by decreased aggregate size and increased single chain content. In this work, the correlation between additives properties, solution state and final film morphology was established. A moderate solubility parameter difference between additives and polymer $\left(4.02 \mathrm{~J}^{1 / 2} \cdot \mathrm{cm}^{-3 / 2}\right)$ and a higher boiling point of additives $\left(266-283{ }^{\circ} \mathrm{C}\right)$ led to the maximum content of single chains $(99.3 \%$ ) and a moderate size of single chains (about $6.0 \mathrm{~nm}$ ). This optimal solution state resulted in the formation of nanofibrils in the spin-coating films from pBTTT solution in oDCB/12-thiol. Apart from the content of single chains, the size of single chains too large or too small was adverse to the formation of fibrils, for the tendency to entangle again due to extended conformation [148] or too shrinking chains inducing excessive aggregation [151], respectively. Therefore, the proper chose of additive is crucial to maximize the content of single chains and control a appropriate size of single chains. In general, the method of adding additives is simple and promising to optimizing the solution state of chains.

A good solvent such as chloroform or chlorobenzene also helped the elevation of single chain content in solution compared with marginal solvent such as tetrahydrofuran (THF) and toluene for PQT12 (Figure 18III) [143], which led to parallel aligned nanofibrils rather than fused bundle-like aggregates disordered in films. Besides, filtration of casting solution through a filter with pore size of several hundreds nanometers could decrease the content of entangled aggregates in solution to promote the fibrillar morphology in films [143], which explained the reason of filtering solution before casting in many works. In summary, providing external energy, entropy control by additives, a rational chose of solvent and removing aggregates by size exclusion are effective methods for disentangment of chains for a further ordered reorganization of chains. 
Figure 18. (I) The ultrasonic control of solution state and morphology for P3HT: (A) the DLS results of P3HT (87 k) solution in p-xylene without and after treatment, (Reprinted with permission from [146]. Copyright 2010 American Chemical Society); (A') the visible diffusion of solute diffusion into solvent for P3HT (low-MW, $35 \mathrm{k}$ ) without and after treatment; (B) the aggregation order of P3HT (68 k for high- $M_{\mathrm{w}}$ and $35 \mathrm{k}$ for low- $M_{\mathrm{w}}$ ) solution with different treatment time; (C) the AFM images for high-Mw P3HT films cast from solution without and after treatment; (D) the device performance variation with the treatment time (Adapted with permission from [147]. Copyright 2013 Wiley-VCH Verlag); (II) The schematic illustration of the role of nonane played in the conformations and aggregation of MEH-PPV chains in DCB solution in (A) and the viscosity variation with adding content of nonane, (Reprinted with permission from [148]. Copyright 2008 American Chemical Society); and (III) The DLS results of PQT12 solutions in different solvents and the TEM images of corresponding films $\left(20{ }^{\circ} \mathrm{C}, 0.1 \mathrm{mg} / \mathrm{mL}\right)$. (Adapted with permission from [143]. Copyright 2013 The Royal Society of Chemistry).
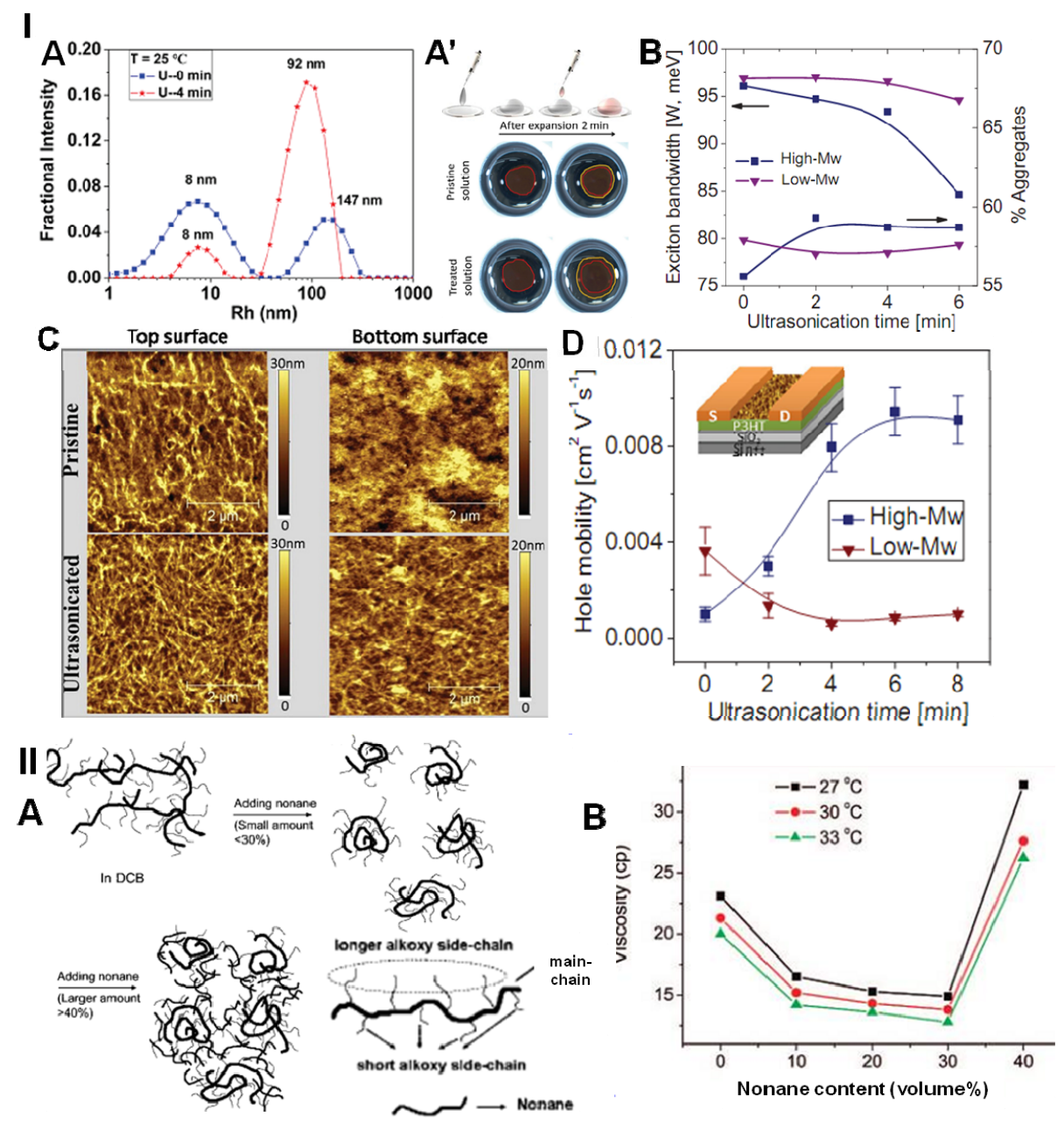
Figure 18. Cont.

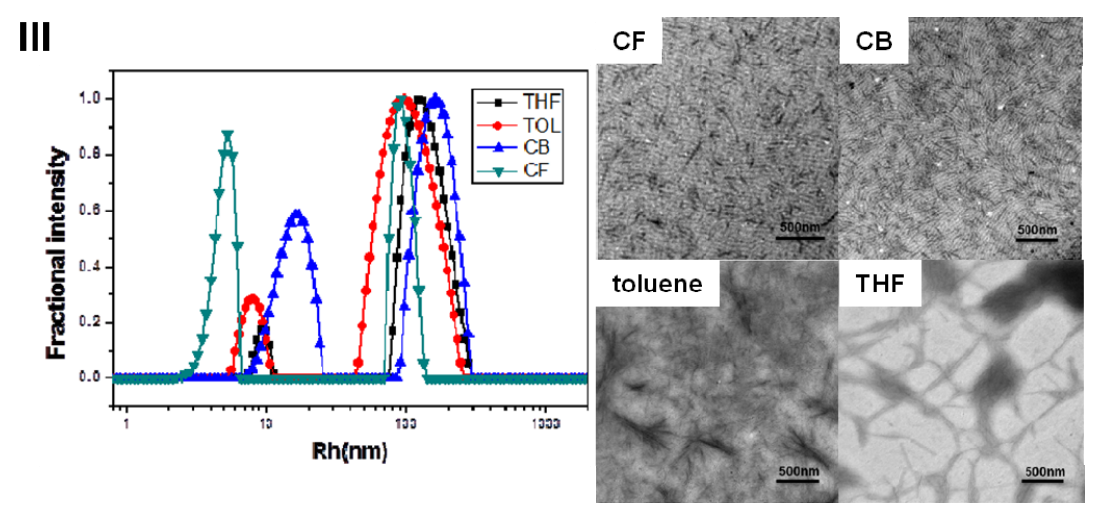

\subsubsection{Promotion of Coil-to-Rod Transition and Ordered Rod-Rod Stacking}

After fulfilling the premise of sufficient content of single chains, the crucial factor is promoting the thermodynamically favored [68] coil-to-rod transition and the following rod-rod stacking. According to the definition of free energy change, $\Delta G=\Delta H-T \Delta S$, there are two pathways to increase the thermodynamic driven force favored by a lower free energy $(\Delta G<0)$ for the coil-to-rod transition with the entropy-declining nature ( $\triangle S<0)$ : (1) $\Delta H$ nearly unchanged but decreasing $T$ and (2) constant $T$ but increasing $|\Delta H|$ with $\Delta H<0$. Reflected by the red-shift and enhanced absorbance at bigger wavelengths in spectra, in situation (1), the straight method is lowering the temperature of the solution to initiate the coil-to-rod transition $[68,138,141,144,152]$, and in situation (2), the transition is induced by adding non-solvent or bad solvent into the solution in a good solvent because of both the minus $\Delta H$ arising from the unexposure of conjugated planes with high surface energy towards solvents to lower the total energy and the increased $|\Delta H|$ arising from the larger difference of solubility parameters between solvents and polymers according to $|\Delta H| \infty(\Delta \delta)^{2}$ [140,153-155]. With the further decrease of temperature or addition of bad or non-solvent, the tendency of coil-to-rod transition is more obvious and the rod-like conformation is more prevailing. Due to the enhanced ordered aggregation initiated by coil-to-rod transition, more charge transport pathways are provided for the improved carrier mobility or PCE of devices.

As to the growth of ordered aggregates by inter-chain $\pi-\pi$ stacking following conformational transition, it leads to a further declining free energy [156] due to the less exposure of high-energy $\pi$-planes to solvents by their face-to-face contact [28]. Meanwhile, the $\pi-\pi$ stacking into ordered aggregates such as nanofibrils is also an entropy-declining process [156]. Therefore, the decreasing of solution temperature and adding bad or non-solvent mentioned above also promote the the $\pi-\pi$ stacking, reflected not only in the enhanced absorbance of lower energy electronic transition in spectra but also by the increased precursor size in solution, fibril length and development of clearer fibrillar morphology in films [152,154]. Besides the driven force, there are two important factors affecting the inter-chain aggregation and final morphology: growth kinetics and the nucleation-growth scheme in solution. Firstly, because of the energy barrier from rod-like single chains to aggregates like nanofibrils [142], the growth of ordered aggregates is a kinetic process. Therefore, aging is an effective way for a fully growth of crystallites in solution, i.e., the increasing amount and length of nanofibrils with longer aging time (Figure 19) and the enhanced $0-0$ and $0-1$ absorbance of solution [145,153,156,157]. He et al. 
reported that even the aligned microwires with the height of $0.8-1.4 \mu \mathrm{m}$, width of $2-4 \mu \mathrm{m}$ and length of $50-1000 \mu \mathrm{m}$ in films were prepared by a rapid solvent evaporation of P3HT solution in anisole aging for 2 months under vacuum $(<133 \mathrm{~Pa})$ for $2 \mathrm{~h}$, and the microwires have a nature of single crystals [158]. Secondly, the growth of crystallites via inter-chain stacking undergoes a nucleation-growth mechanism. The nucleation stage plays a determining role in the final morphology according to the order, shape and density of nucleates [77,152,155,159]. An effective strategy to promote the fully crystallization of conjugated polymer chains based on nucleation is self-seeding [53,75,77,152,159]. Self-seeding means the homogeneous formation of nucleates in the supersaturated solution at a relatively higher temperature $\left(T_{\mathrm{ss}}\right)$ preventing excessive aggregation followed by a further ordered growth of nucleates into crystals with a larger size at a constant lower temperature $\left(T_{\mathrm{c}}\right)$. The chose and stability of solution temperature $\left(T_{\mathrm{ss}}\right.$ and $\left.T_{\mathrm{c}}\right)$ is crucial for the success of crystal preparation. Rahimi et al. reported the preparation of P3HT single crystals with a high density via self-seeding processing of P3HT solutions in 3-hexylthiophene with a very concise of temperature $\left( \pm 0.1^{\circ} \mathrm{C}\right)[77]$. The higher the seeding temperature $\left(T_{\mathrm{ss}}\right)$ was, the lower nucleation density and the larger size of needle-like crystals in the final films were (Figure 20a). However, the tiny variation of $T_{\mathrm{ss}}$ led to a remarkably change of nucleation density. Oh et al. [152] reported a self-seeding method with a reversed sequence, that is, cooling at $-20^{\circ} \mathrm{C}$ to form longer nanofibrils and then heating to $25^{\circ} \mathrm{C}$ to form homogeneous crystal seeds with smaller sizes (Figure 20b). They also considered the influence of solubility parameter difference between solvents and polymer $(\Delta \delta)$ in this self-seeding processing. With the larger $\Delta \delta$, the nucleation temperature turned higher, which meaned a larger supercooling when self-seeding $\left(25^{\circ} \mathrm{C}\right)$ and led to a higher crystallinity in solution and more and longer nanofibrils as charge transport pathways in corresponding films (Figure 20b'). When $\Delta \delta$ larger than 0.7 , the relative crystallinity in dilute solution was maximal (0.56). Similar to the spin-coating process, with the increasing concentration, the relative crystallinity in solution with $\Delta \delta$ larger than 0.7 reached nearly 1.0 , which resulted in the improvement of the charge transport $\left(-9.80 \mathrm{~mA} / \mathrm{cm}^{2}\right)$ in corresponding films and PCE (3.525\%) of solar cells. Yu et al. combined the effect of both temperature controlled nucleation and sonication to enhance content of free single chains [53]. By elevating the solution temperature, the content of nucleates decreased due to dissolution and the content of free single chains increased due to heating and sonication, leading to the growth of a considerable number of longer nanofibrils (from 135 to $559 \mathrm{~nm}$ ) in final films to improve the charge transport and PCE of solar cells (Figure 20c). Apart from self-seeding, a second nucleation-growth scheme is subsequent different nucleation. Yan et al. reported the formation of branched and hyperbranched P3HT nanostructures from P3HT solutions in oDCB/trimechanolamine (TEA) [155]. The additive, TEA, was a bad solvent for P3HT with a very high boiling point. At the early stage of solvent evaporation, the role of oDCB as a good solvent was prevailing and homogeneous nucleation happened for P3HT to form long nanowires slowly. At the late stage of evaporation, the role of TEA was prevailing and promoted the aggregation of chains. The trunk-like nanowires played a role as sites for heterogeneous nucleation. As a result, the branched and hyperbranched nanostructures were tuned by the ratio of oDCB and TEA (Figure 21). A third nucleation-growth scheme is dispersed nucleation and assisted growth in phase separation room [160-164]. Through mixing the conjugated polymers with insulting flexible polymers with a small content of conjugated polymers, i.e., polystyrene, the nucleates are dispersed in the matrix of flexible polymers due to phase separation and grow further to 
form percolation pathways for charge transport at a low percolation threshold (1.7 vol \% [162]). Broadly, the matrix of insulting polymers could be regarded as solid "solvent" for aggregates of conjugated polymers. Lu et al. reported the effects of the crystalline properties of flexible polymers on aggregation of conjugated polymers in blend films [160]. For amorphous flexible polymers, the nanofibrils formed were homogeneously dispersed by the vetrificated matrix, leading to a maximized semiconductor /insulator interface. On the contrary, the (semi-)crystalline flexible polymers formed domains with a large size and excluded the originally dispersed nanofibrils out of crystalline regions to form bundle-like submicro-wires, resulting in the reduced interface. A larger semiconductor/insulator interface accounted for the better charge transport in blends with a amorphous insulting polymer such as PS (Figure 22I). Kim et al. discussed the influence of content of P3BT on the morphology and carrier mobility in blend films with PS [162]. Although the effective carrier mobilities for films with different blend ratios did not differ a lot, the mobility in nanowires increased with reducing content of P3BT and coverage of nanowires on the surface. When more P3BT was added in blend, the density of nanowires and the inter-hopping frequency were higher and the effective transport length in nanowires was reduced. It led to the inefficient inter-nanowire transport prevailing. On the contrary, a low content of P3BT led to the prevailing of intra-nanowire transport as high as $0.12 \mathrm{~cm}^{2} \cdot \mathrm{V}^{-1} \cdot \mathrm{s}^{-1}$ in the films due to fewer inter-nanowire contacts, which was promising for applications in devices. Recently, Benetti et al. have developed the assisted aggregation of P3HT in the cages of flexible polymer gels without any solvent during cooling (PMMA) [161]. The template effect of cages with a proper size in the gel with a low cross-linking degree ( $2 \%$ ) led to the formation of dispersed nanocrystals in gel films with a free exciton of $31 \mathrm{meV}$ much smaller than those prepared from solution in anisole $(108 \mathrm{meV})$, reflecting the much longer conjugation in crystals and a better order (Figure 22II). A high cross-linking degree prolonged and hampered the ordered aggregation of P3HT due to the too small cages in gels. Thus, the methods of blending with insulating flexible polymers are proven promising in future device processing due to both the enhanced order and performances of films and the saving of conjugated polymers.

Figure 19. TEM images showing growth process of P3DDT nanowhiskers drop-cast from P3DDT/CB-anisole $(1: 4,0.1 \mathrm{mg} / \mathrm{mL})$ solution aged for different times, (Adapted with permission from [156]. Copyright 2011 American Chemical Society).

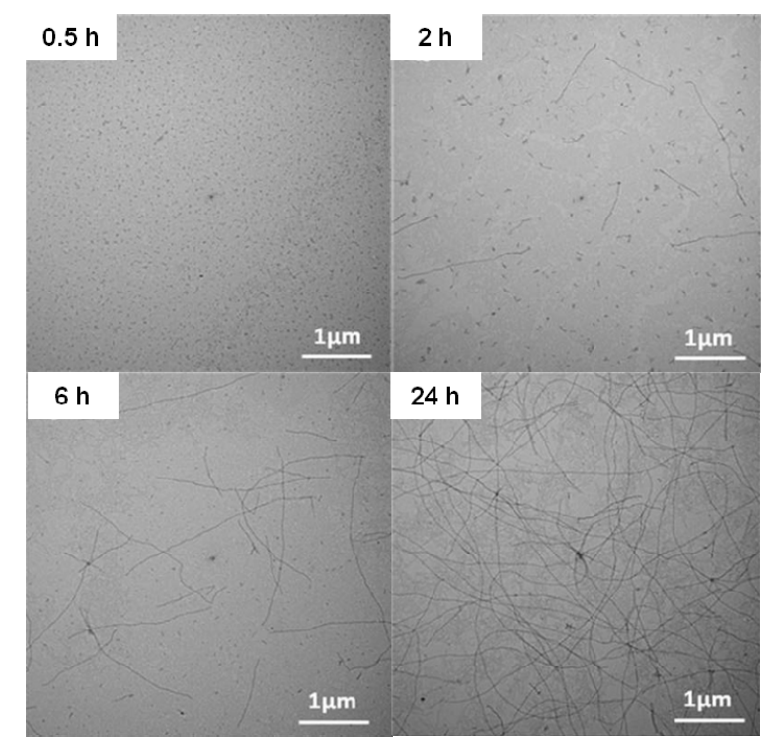


Figure 20. (a) The influence of $T_{\mathrm{ss}}$ on the crystallization morphology (optical microscopy images with a range of $100 \times 100 \mu \mathrm{m}^{2}$ ), seed density, crystal length and crystal density (Adapted with permission from [77]. Copyright 2012 Wiley-VCH Verlag); The influence of solution temperatures on the solution state and film morphology during self-seeding in (b) and the influence of solubility parameter difference between P3HT and solvent on the morphology of films cast from solutions according to the same self-seeding procedures in (b'), (Adapted with permission from [152]. Copyright 2012 American Chemical Society); and (c) The influence of temperatures of solution during sonication on aggregation in solution and fibril length and the TEM images of films cast from solution undergoing sonication at different temperatures (scale bar: $500 \mathrm{~nm}$ ) (Adapted with permission from [53]. Copyright 2012 American Chemical Society).
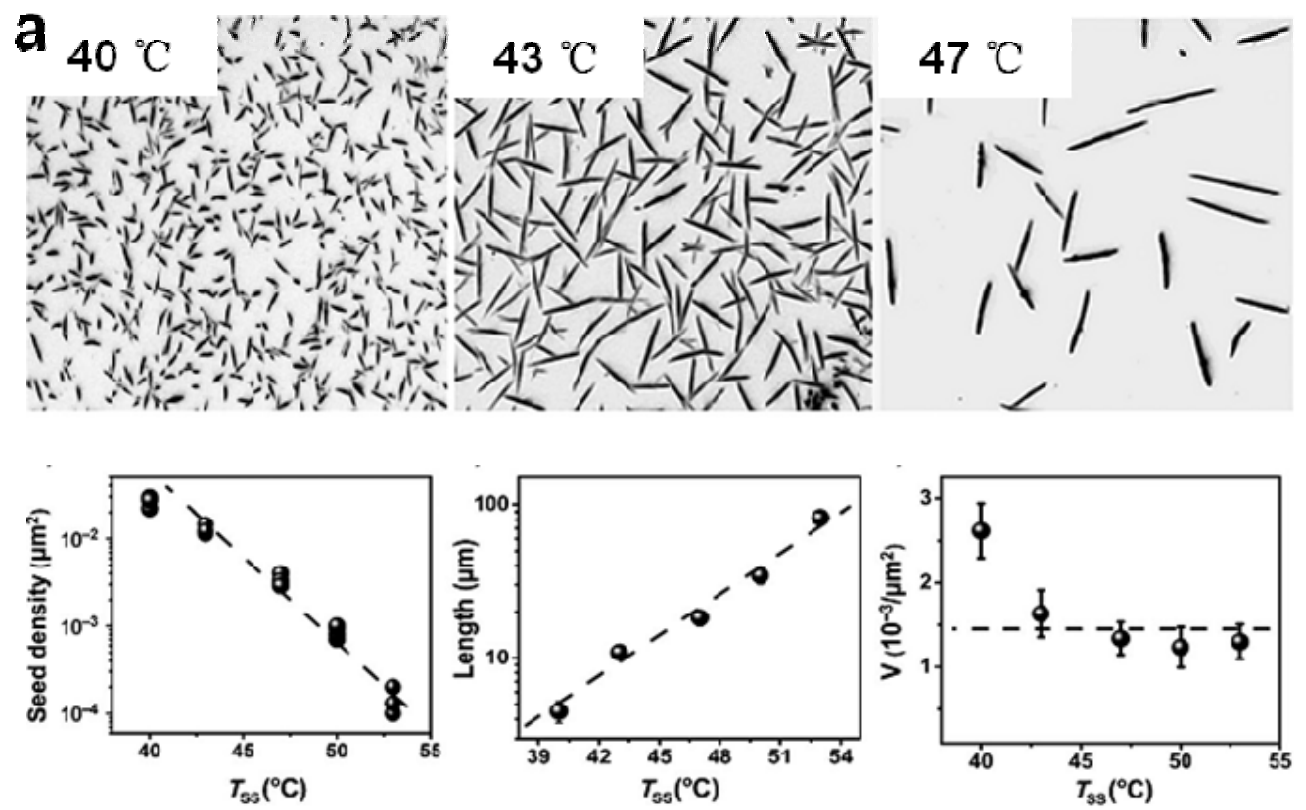
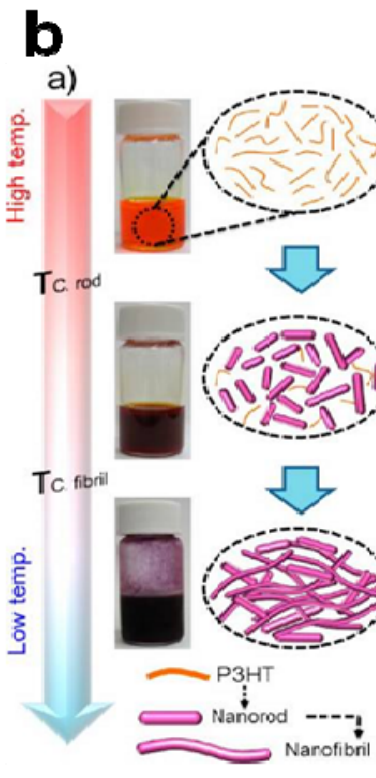
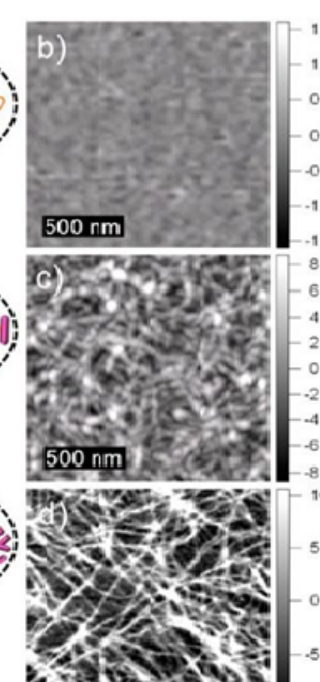

b'

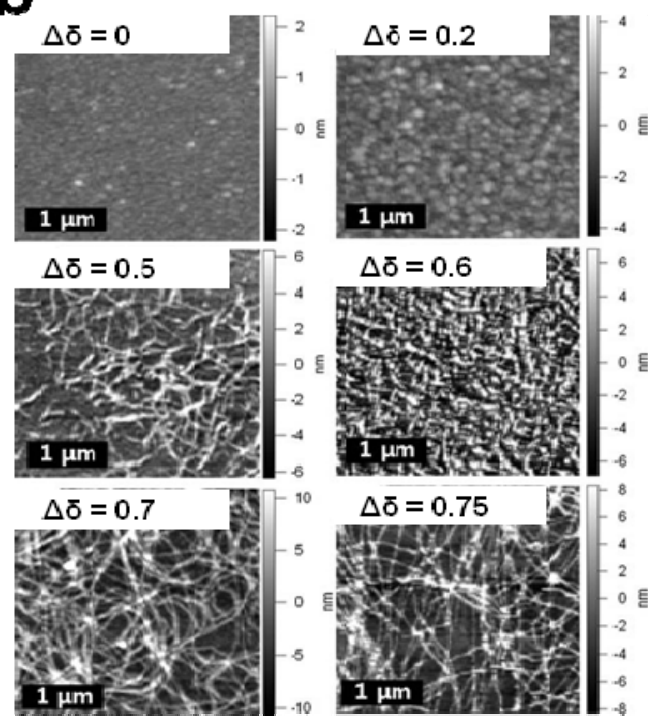


Figure 20. Cont.
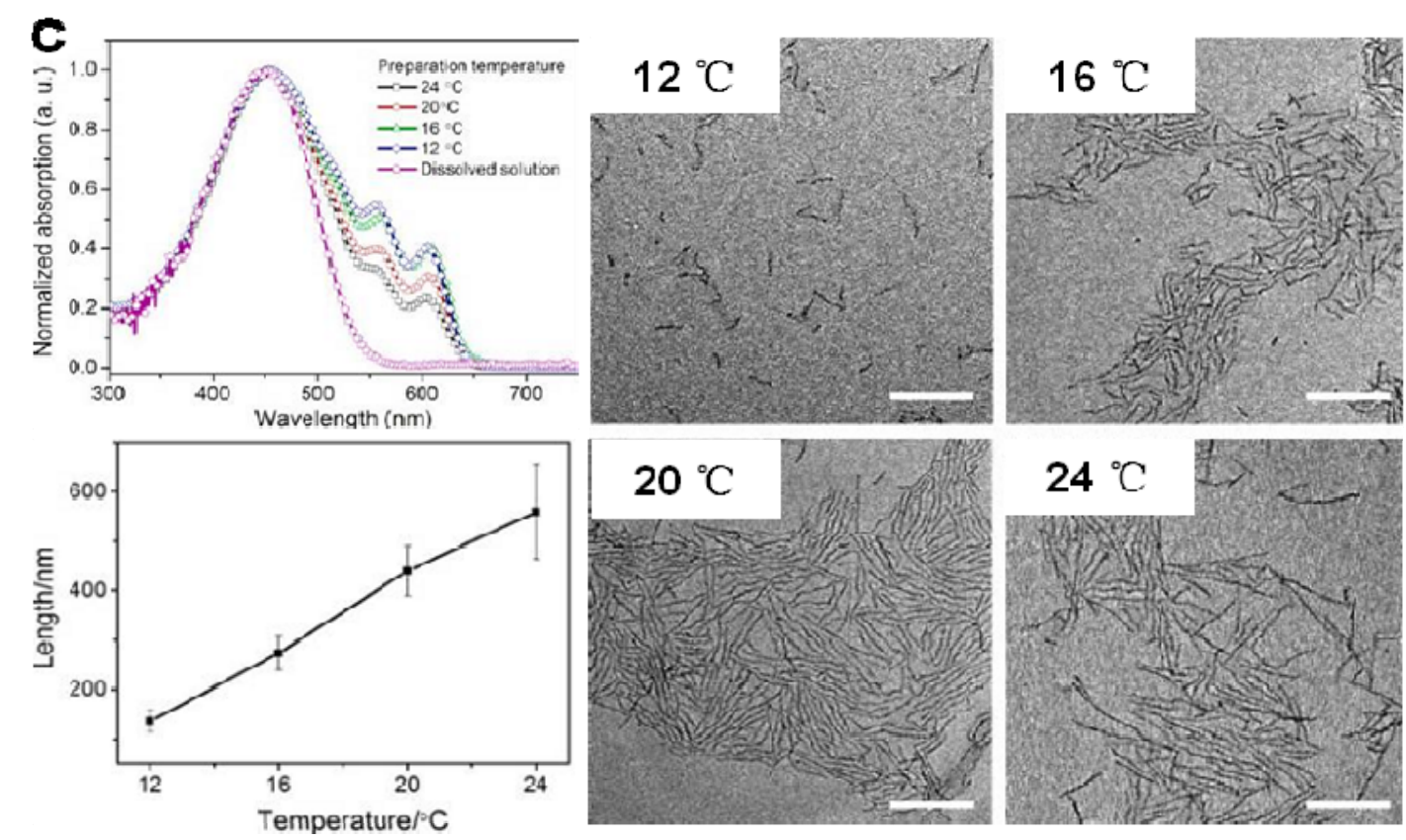

Figure 21. (a) The UV-Vis absorption spectra of P3HT solution in dichlorobenzene (DCB)/trimechanolamine (TEA) mixture with different TEA/DCB volume ratio and (b) the time-dependent evolution morphology of adding a $30 \mu \mathrm{L}$ TEA solution and the growth mechanism of P3HT branched nanostructures, (Adapted with permission from [155]. Copyright 2011 American Chemical Society).
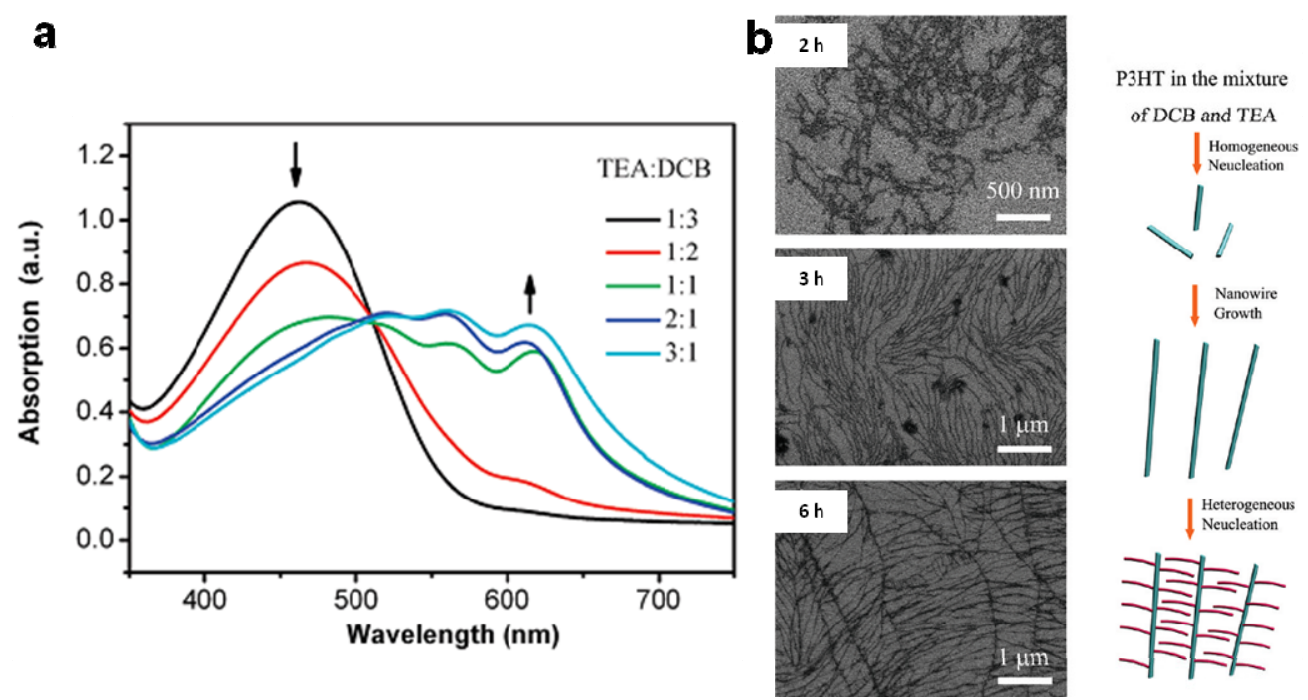
Figure 22. (I) Schematic illustration of the morphology evolution of P3BT/insulating polymer composites prepared from solution, employing amorphous and crystalline matrix polymers, respectively and the dependence of electrical conductivity $\sigma$ of P3BT composites on P3BT content at room temperature, (Adapted with permission from [160]. Copyright 2010 Wiley-VCH Verlag); (II) The schematic illustrations of preparation of P3HT nanocrystals in a PMMA gel in (A); UV-Vis absorption spectra of P3HT nanocrystals in anisole solution (solid line) and in PMMA gels at different crosslinker content with the inset showing the calculated exciton bandwidth $(W)$ in $(\mathbf{B})$; and the tapping-mode AFM phase micrographs showing P3HT aggregates formed in PMMA2 or in anisole solution in (C), (Adapted with permission from [161]. Copyright 2012 Wiley-VCH Verlag).
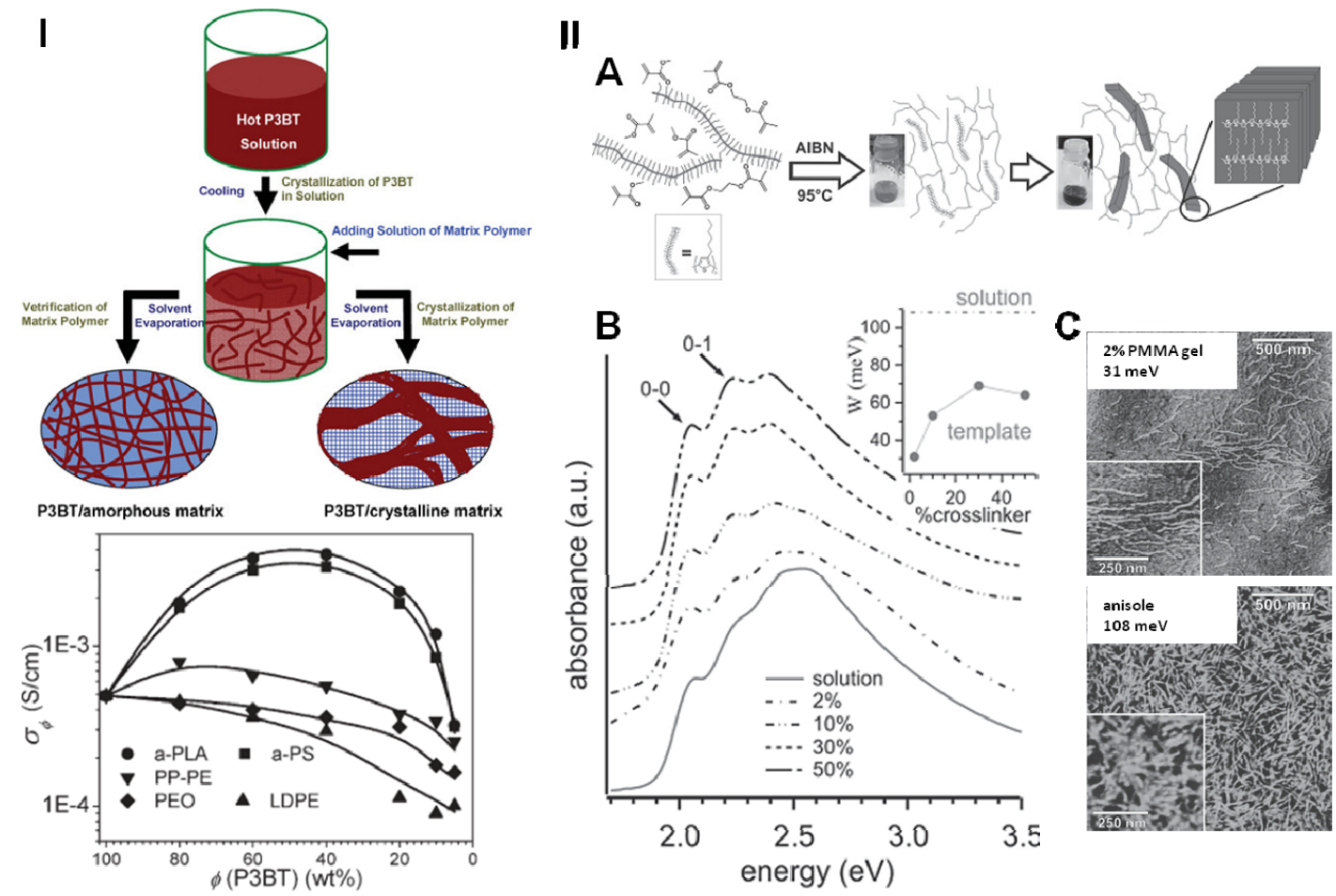

Besides thermodynamic factors, the fully growth of ordered aggregates, i.e., crystals, depends closely on the assembly time for chains for the barrier to a more stable equilibrium state. Besides aging as an efficient way to promote ordered aggregation of chains in solution before casting, it is hoped that the ordered aggregation could be maximized straightly during casting as a simple processing free of aging. Thus, the essential factor in the kinetic control over morphological features is tuning self-assembly time via controlling evaporation rate of solvent. In general, a slow evaporation rate is favored for the formation and growth of crystals in films. Kim et al. reported that the gradual growth of aggregates of P3HT from few nucleates to a large amount of long nanowires during spin-coating via reducing evaporation rate of chloroform tuned by a larger vapor pressure of chloroform in atmosphere (Figure 23a) [165]. Chang et al. realized the formation of P3HT nano-ribbon with a diameter of 30-50 nm in film by using a good solvent with a extremely high boiling point $\left(219^{\circ} \mathrm{C}\right), 1,2,4$-trichlorobenzene, to largely reduce the evaporation rate of solvent (Figure 23b) [166]. The carrier mobility of film spin-coated from solution in TCB was $0.12 \mathrm{~cm}^{2} \cdot \mathrm{V}^{-1} \cdot \mathrm{s}^{-1}$, which was much higher than that from solution in chloroform. Wang et al. reported the formation of longer nanofibrils of pBTTT in films 
simply by reducing the spin-coating rate to increase self-assembly time [150]. Meanwhile, the reduced entanglement of nanofibrils indicating a more ordered morphology was obtained using a slower spin-coating rate, which was attributed to the equilibrium favored by more single chains for nanofibril formation and less disordered aggregates as entanglement points due to delayed crystallization of chains. However, a too slow evaporation of solvent by drop-casting resulted in the fusing of fibrils again, indicating the nature of a favored kinetic equilibrium state for dispersed long fibrils (Figure 23c). Compared with the nanofibril morphology in nanoscale obtained by "slow" spin-coating, a much slower evaporation rate of solvent in a sealed room helps the formation of crystals with larger sizes in micro scale [73,74,78,167-170]. Apart from extremely slow evaporation, factors such as the properties of substrate [167], temperature [73,167], solution concentration $(<1.0 \mathrm{mg} / \mathrm{mL})$ and solvent composition [74] are related to the formation of micro-sized crystals in films. Thus, the conditions for preparing micro-sized crystals are strict and need concise control, for the formation of them could be easily disturbed by other less stable states with a smaller barrier. These micro-sized crystals display diffraction dots in selected area electron diffraction (SAED) patterns, indicating the single crystal nature of them as the most thermodynamic stable state of chains proven also by the unchanged crystalline structures and morphology for crystals after thermal annealing [76]. With the perfect order in crystals and proper orientation of chains, the carrier mobilities in these crystals were very high $[73,78,167,170]$ and a max value reached $6.0-7.0 \mathrm{~cm}^{2} \cdot \mathrm{V}^{-1} \cdot \mathrm{s}^{-1}$ in poly(dithieno[3,2-b:2',3'-d] thiophene-alt-diketopyrrole[3,4-c]pyrrole) (PDTTDPP) copolymer single fibers [78]. Thus, preparation of micro-sized crystal by concisely tuning evaporation rate and other factors is promising for higher transistor performances and a better understanding of crystallization behavior of conjugated polymer chains.

Figure 23. (a) Tapping mode scanning force microscope (TM-SFM) images $\left(2 \times 2 \mathrm{~mm}^{2}\right)$ of P3HT molecular nanowires deposited on a substrate $\left(\mathrm{SiO}_{x}\right)$ at different vapor pressures (Adapted with permission from [165]. Copyright 2005 Wiley-VCH Verlag); (b) Tapping-mode AFM images of P3HT film made from solutions in chloroform and TCB in a range of $1 \times 1 \mu \mathrm{m}^{2}$ (Adapted with permission from [166]. Copyright 2004 American Chemical Society); and (c) The TEM images of films coated from PBTTT-12 solutions in ODCB/5\% 12-thiol mixture at different rates (scale bar: $5 \mu \mathrm{m}$ ) (Adapted with permission from [150]. Copyright 2013 Elsevier).
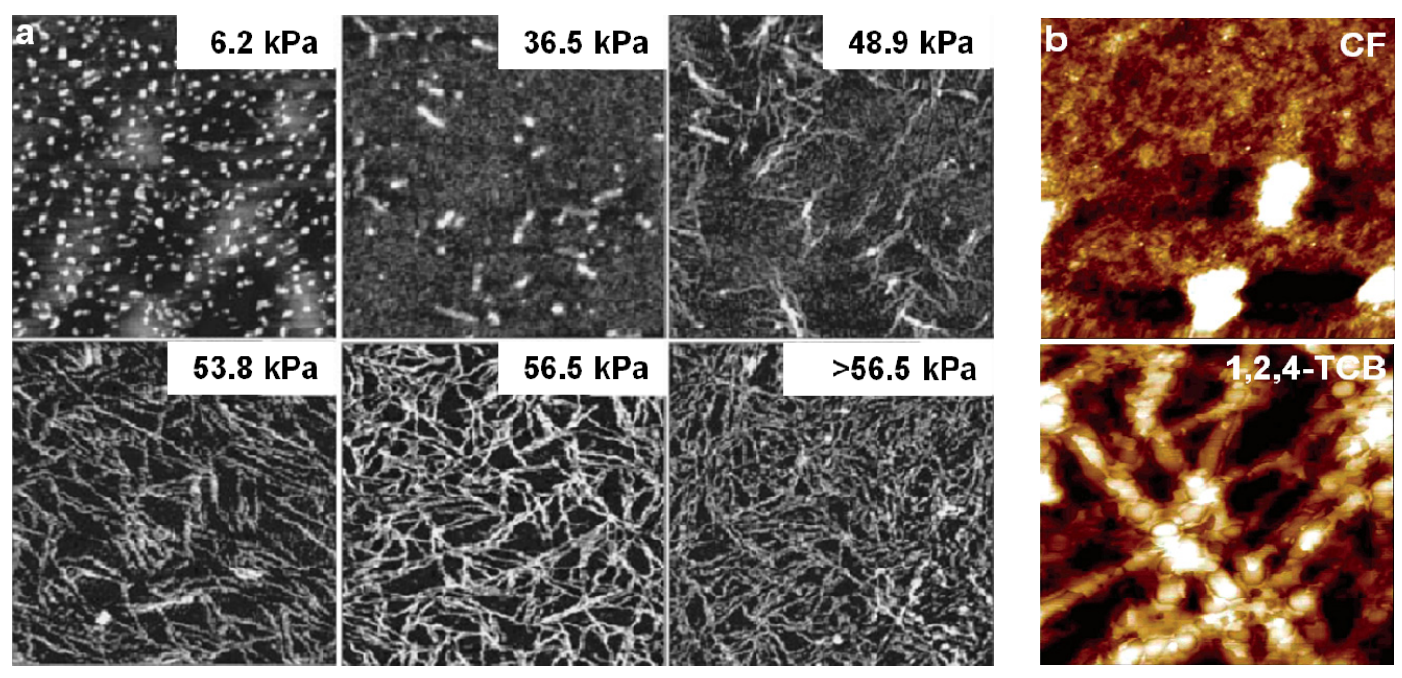
Figure 23. Cont.

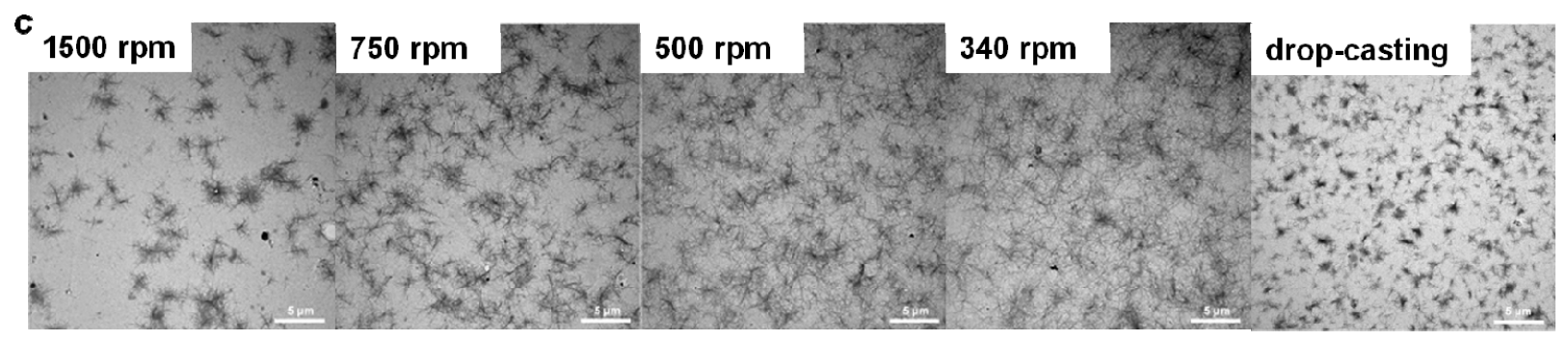

According to the discussions above, a scheme for optimizing the solution state is proposed as subsequent disentanglement, promotion of both coil-to-rod transition and rod-to-rod stacking following, and tuning the nucleation-growth process. Combining all these strategy into a systematic program for tuning ordered aggregation of conjugated polymers for charge transport in solution will be helpful for the straight processing of solution to prepare efficient film devices. The strategy of solvent vapor annealing is mild [171] and flexible due to the treatment at room temperature and various solvent vapor atmospheres. As a method involving (partly) dissolution of chains, SVA is also a potential tool simulating solution processing to better understand the self-assembly of conjugated polymers during film formation. Basically, there are two stages during the solvent vapor annealing of films, solvation regime and aggregation regime [172]. According to the Ostwald ripening equation, the critical size at which stable aggregates can form, $\mathrm{RC}$, is defined as follows,

$$
R_{\mathrm{C}}=\left(\frac{2 \sigma}{k T}\right) v \frac{C_{\infty}}{C_{\mathrm{CP}}-C_{\infty}}
$$

In Equation (4), $\sigma$ is the interphase surface tension between solute and solvent, $v$ is the atomic or molecular volume of solute, $C_{\mathrm{CP}}$ is the solute concentration gradually changing during annealing, $k$ is Boltzmann constant, $T$ are absolute temperature and $C_{\infty}$ is an intrinsic property of one polymer as the saturation limit of the solute concentration at which chain entanglement and hence gelation occurs. When $C_{\infty}>C_{\mathrm{CP}}$ at the early stage of annealing, solvation prevails and when $C_{\infty}<C_{\mathrm{CP}}$ at the late stage of annealing, aggregation prevails.

In the solvation regime, the intrachain interchromophoric interactions are dominating and the original rod-like folding chains are unfolded (more coil-like) and more free to move reflected by the increased diffusion velocity and fluorescence intensity of chains in single-molecule fluorescence excitation polarization spectroscopy, which is thought important for the formation of ordered aggregates (Figure 24I) [136,137]. Thus, a smaller solubility parameter difference between solvent and solute determining a good solubility is important to satisfy the premise of ordered aggregation and enhance performances. Liu et al. and Wang et al. reported that with closer solubility parameters of solvent to polymer, the crystallinity and/or fibrillar morphology in films became improved [79,173]. Meanwhile, with the similar solubility parameters, a larger polarity of solvent led to a drop of carrier mobility. Besides, the larger vapor pressure could result in a better solvation of chains and formation of ordered aggregates such as nonofibrils [79]. In the following aggregation regime, the inter-chain interactions are dominating and the chains reorganize via folding and aggregation again in another way. Many morphological and structural features, i.e., crystal morphology, size and density of crystalline regions, and crystalline structure and order etc. are fixed in this regime influenced by factors such as solvent 
quality, vapor pressure and treatment time, etc. Firstly, the quality of solvent or vapor atmosphere is important due to its determining on $C_{\infty}$ and nucleation [79,159,172]. For a bad solubility of solute arising from larger conjugation lengths, bad solvent or smaller vapor pressure, the value of $C_{\infty}$ is small leading to a small size and larger density of crystallites or even no nucleates. However, for a too high solubility in some good solvent, $C_{\infty}$ is too high to form nucleates for their infinitely large size. Therefore, to obtain ordered crystalline aggregates or domains with a larger size and fewer grain boundaries, a properly good solvent (Figure 24II) and high vapor pressure (Figure 24III) is needed to form large-sized nuclei. Especially for conjugated polymers with larger conjugation lengths, Wang et al. reported that (1) the critical nucleation vapor pressure was higher due to the higher critical vapor pressure for rod-to-coil transition during solvation and (2) the fibril density was reduced due to the earlier coil-to-rod transition and saturation for growth and slower motion of units during aggregation, which were the results of the enhanced rigidity and smaller room for conformational change arising from larger conjugation lengths for polymers (Figure 7) [79]. Thus, a good solubility during solvation and a later coil-to-rod transition during solidification is found universally important for the preparation of ordered aggregates such as nano-fibrils for conjugated polymers with long conjugated lengths by the tool of solvent vapor annealing. Secondly, the crystallization kinetics for growth after nucleation could also influence the crystalline region size. Crossland et al. reported that a higher crystallization vapor pressure was helpful for the increase of crystalline region size and alignment order of nanofibrils due to the smaller supercooling and slow crystallization kinetics [159]. Xiao et al. observed the gradually increasing length of P3HT needle-like crystals with longer treatment time in THF atmosphere [73]. Liu et al. did a systematic research into the influence of treatment time on the morphology and crystallinity [173]. An optimal treatment of $24 \mathrm{~h}$ was fixed due to the best connectivity between fibrillar domains and the highest crystallinity. A too long treatment ( $>48 \mathrm{~h}$ ) led to excess disordered aggregation and worse connectivity in morphology and a drop in crystallinity. In summary, by optimizing important parameters such as the solvent quality, vapor pressure and treatment time according to the intrinsic molecular structure, one can obtain excellent morphology and performances by solvent vapor annealing. Considering the significant improvement of morphological order by solvent annealing in pure polymer film, a mixed vapor atmosphere made of solvent interacting well with polymer and fullerene respectively has been employed to tune both the good phase separation and continuous pathways of polymer fibrillar structures and improve the power conversion efficiency (from $4.54 \%$ to $6.60 \%)$ in poly[[9-(1-alkyl)-9H-carbazole-2,7-diyl]-2,5-thiophenediyl-2,1,3benzothiadiazole-4,7-diyl-2,5-thiop-hene-diyl] (PCDTBT)/[6,6]-phenyl-C ${ }_{71}$-butyric acid methyl ester $\left(\mathrm{PC}_{71} \mathrm{BM}\right)$ solar cells [174]. Therefore, the strategy of solvent vapor annealing is very promising in the processing of devices due to its flexibility and versatility.

At last, it is noted that the control over the development of thermodynamic state of the system is crucial to the formation of optimal morphology. "Insufficient" and "excess" are two problems existing in the control over structures and morphology in films. Literally, "insufficient" means that there is room to reach the optimal state and "excess" means that the optimal state is climbed over followed by a state no better. When tuning the structures and morphology in films, the insufficient driving force such as a fast coating rate [64], a low annealing temperature [38,39], a low annealing solvent vapor pressure [79,159] or a short treatment time [173] could result in the morphology not developing fully reflected by less fibrillar features or more fused shorter nanofibrils, etc. However, a excess treatment 
such as too slow drying rate [150], too long treatment time [156,173] or too high annealing temperature [38,39] lead to the destruction of the original optimal state reflected by reduced crystallinity, less ordered fibrillar features and fusing of nanofibrils again, etc. Therefore, achieving the state of system with an optimal morphology suitable for devices is factually the target in practical processing and post-treatments, regardless of the kinetic or thermodynamic equilibrium nature of this optimal state.

Figure 24. (I) Fluorescence intensity transient of a single MEH-PPV chain within a PMMA matrix without and during SVA: (a) wide-field fluorescence images corresponding to the fluorescence transient in (b) at the times indicated and (b) the complete fluorescence transient and the velocity; (c) scheme of the relation between the conjugated polymer morphology, brightness, and solvent vapor annealing; (II) The schematic illustrations of solvent quality on the aggregation size in (A) and the corresponding morphological and spectroscopic evidences in (B), (Adapted with permission from [172]. Copyright 2012 American Chemical Society); (III) The control by vapor pressure over the crystalline region or crystal sizes for P3HT spherulites (A) (Adapted with permission from [159]. Copyright 2010 Wiley-VCH Verlag) or PQT12 (15.5 k) nanofibrils (B, scale bar: $500 \mathrm{~nm}$ ), (Adapted with permission from [79]. Copyright 2013 American Chemical Society).

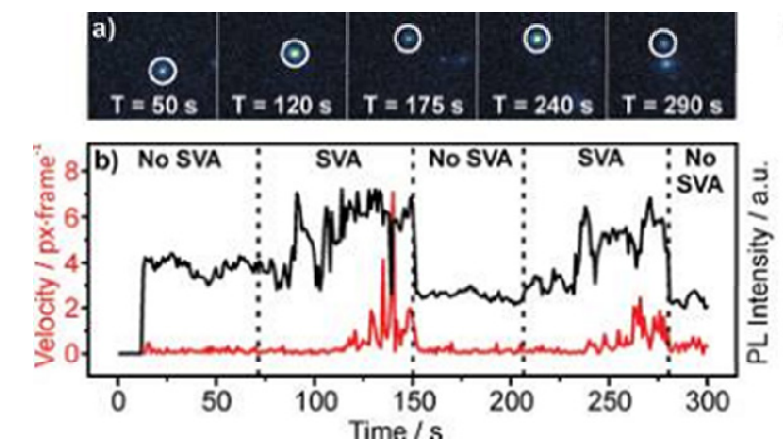

c)

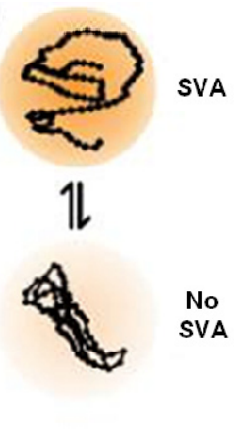

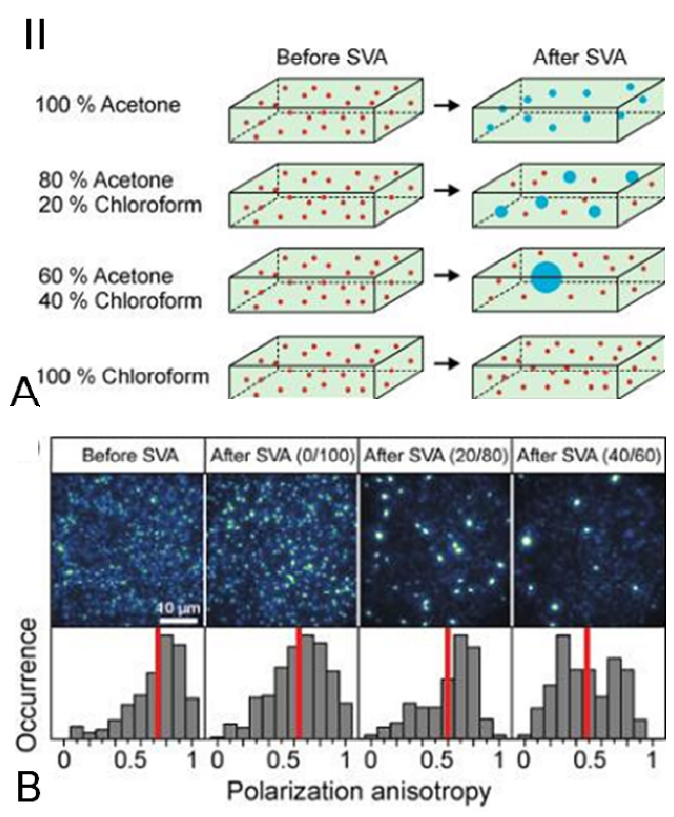

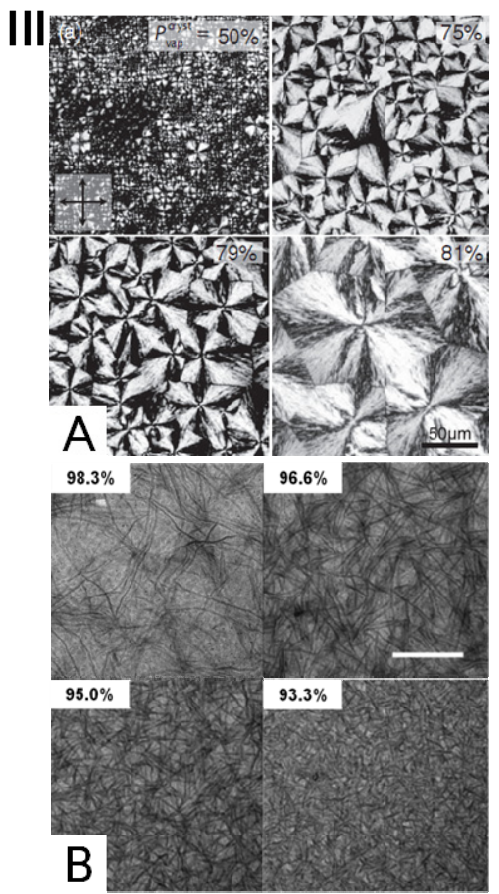


In summary, in this section a scheme for optimizing the solution state is proposed as subsequent disentanglement, promotion of both coil-to-rod transition and rod-to-rod stacking following, and tuning the nucleation-growth process. Combining all these strategy into a systematic program for tuning ordered aggregation of conjugated polymers for charge transport in solution will be helpful for the straight processing of solution to prepare efficient film devices (Figure 25).

Figure 25. Schematic illustration of the control over solution state and casting films to obtain ordered morphology: procedures, strategies and parameters.

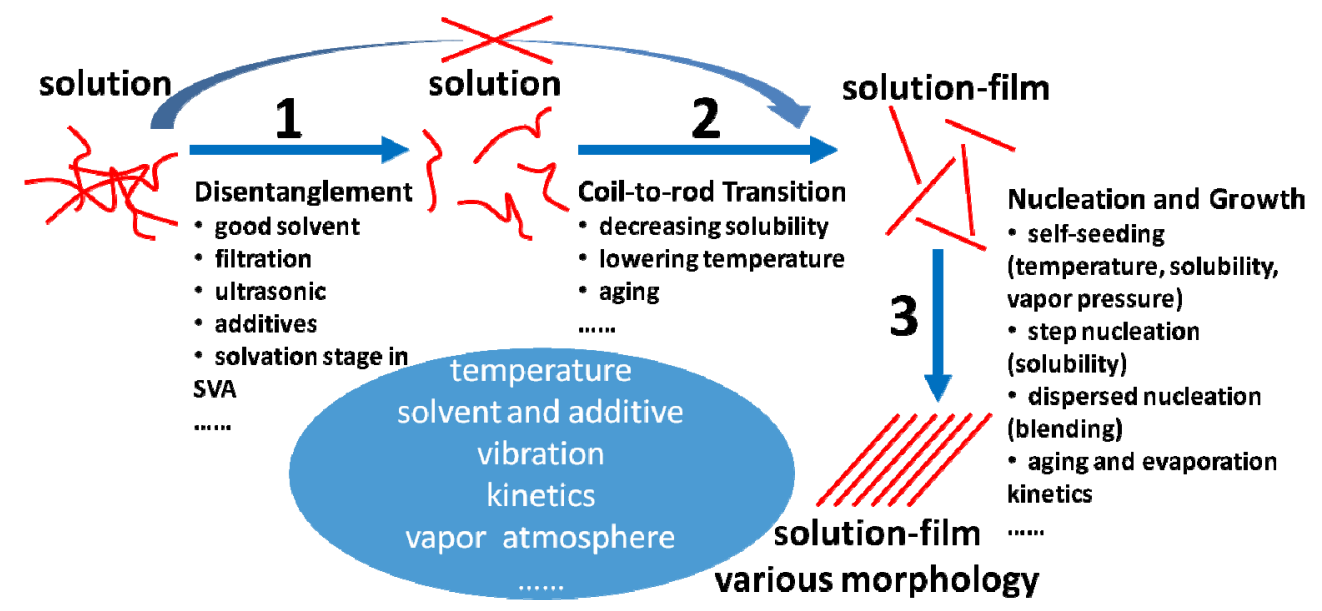

\section{Concluding Remarks and Outlook}

In this review, the crucial morphological and structural features dominating the charge transport in film as the most essential origin for a better device performance of semiconductor conjugated polymer are summarized and demonstrated in terms of both theory and applications, that is, intra- and inter-grain order, crystallinity and alignment. By summarizing the recent research into polymer-based organic electronics especially in the last five years, the ways to tune and obtain a better morphology and electronic property of functional films are listed in terms of material chose and design, solution state optimization and processing strategies for solution and films, which are the three important facets for the development of this field in both lab and industrial applications. Firstly, by analyzing the effect of intrinsic molecular structure on the bottom-up physical features, it is no doubt that the development of new-generation materials with more extended conjugation structures, more proper chains shapes and more ordered self-assembly is the origin of enhanced property. Secondly, optimizing the solution state of polymers from single chains to maximal formation of ordered aggregates is the efforts needing to be made for the preparation of efficient films direct from solution because of the out-standing solution-processable advantage. Thirdly, the utilization of conventional solution processing or post-treatment strategy to prepare more ordered crystalline structures like aligned nanofibrils or single-crystals and the integration of them into industrial process like roll-to-roll are an important strategy to realize the applications of the excellent electronic properties. When tuning morphology and structure, many parameters associated with solution state, thermodynamic driving forces, kinetics, the preferred devices architectures and even the molecular structural factors of materials, and the trade-off between these parameters need to be considered and tested, which are always challenging but crucial as listed in this review. At last, it is hoped that this review is helpful to guide the material design and 
morphological, structural control of semiconductor conjugated polymers with a brilliant prospect in science and applications.

\section{Acknowledgments}

This work was subsidized by the National Natural Science Foundation of China (21334006, 51273191, 51073151, 51303177) and Scientific Development Program of Jilin Province (20130101007JC).

\section{Conflicts of Interest}

The authors declare no conflict of interest.

\section{References}

1. Wang, C.L.; Dong, H.L. Semiconducting pi-conjugated systems in field-effect transistors: A material odyssey of organic electronics. Chem. Rev. 2012, 112, 2208-2267.

2. Kim, F.S.; Ren, G.Q. One-dimensional nanostructures of pi-conjugated molecular systems: Assembly, properties, and applications from photovoltaics, sensors, and nanophotonics to nanoelectronics. Chem. Mater. 2011, 23, 682-732.

3. Facchetti, A. П-conjugated polymers for organic electronics and photovoltaic cell applications. Chem. Mater. 2011, 23, 733-758.

4. Mei, J.G.; Diao, Y. Integrated materials design of organic semiconductors for field-effect transistors. J. Am. Chem. Soc. 2013, 135, 6724-6746.

5. AlSalhi, M.S.; Alam, J. Recent advances in conjugated polymers for light emitting devices. Int. J. Mol. Sci. 2011, 12, 2036-2054.

6. Tang, C.; Liu, X.D. Recent progress in polymer white light-emitting materials and devices. Macromol. Chem. Phys. 2013, 214, 314-342.

7. Heeger, A.J. Semiconducting polymers: The third generation. Chem. Soc. Rev. 2010, 39, 2354-2371.

8. Krebs, F.C. Polymer solar cell modules prepared using roll-to-roll methods: Knife-over-edge coating, slot-die coating and screen printing. Solar Energy Mater. Solar Cells 2009, 93, 465-475.

9. Amb, C.M.; Craig, M.R. Aesthetically pleasing conjugated polymer: Fullerene blends for blue-green solar cells via roll-to-roll processing. ACS Appl. Mater. Interfaces 2012, 4, 1847-1853.

10. Yue, W.; Larsen-Olsen, T.T. Synthesis and photovoltaic properties from inverted geometry cells and roll-to-roll coated large area cells from dithienopyrrole-based donor-acceptor polymers. J. Mater. Chem. A 2013, 1, 1785-1793.

11. Nielsen, C.B.; Turbiez, M. Recent advances in the development of semiconducting DPP-containing polymers for transistor applications. Adv. Mater. 2013, 25, 1859-1880.

12. Usta, H.; Facchetti, A. $N$-channel semiconductor materials sesign for organic complementary circuits. Acc. Chem. Res. 2011, 44, 501-510.

13. Sista, P.; Biewer, M.C. Benzo[1,2-b:4,5-b']dithiophene building block for the synthesis of semiconducting polymers. Macromol. Rapid Commun. 2012, 33, 9-20. 
14. Huo, L.J.; Hou, J.H. Benzo[1,2-b:4,5-b']dithiophene-based conjugated polymers: Band gap and energy level control and their application in polymer solar cells. Polym. Chem. 2011, 2, 2453-2461.

15. Balan, A.; Baran, D. Benzotriazole containing conjugated polymers for multipurpose organic electronic applications. Polym. Chem. 2011, 2, 1029-1043.

16. Liu, Y.; Liu, Y.Q. High-mobility conjugated polymers based on fused-thiophene building blocks. Macromol. Chem. Phys. 2011, 212, 428-443.

17. Kang, I.; Yun, H.J. Record high hole mobility in polymer semiconductors via side-chain engineering. J. Am. Chem. Soc. 2013, 135, 14896-14899.

18. Lu, L.Y.; Luo, Z.Q. Cooperative plasmonic effect of Ag and Au nanoparticles on enhancing performance of polymer solar cells. Nano Lett. 2013, 13, 59-64.

19. Salleo, A.; Kline, R.J. Microstructural characterization and charge transport in thin films of conjugated polymers. Adv. Mater. 2010, 22, 3812-3838.

20. Park, Y.W. Editorial for the conducting polymers for carbon electronics themed issue. Chem. Soc. Rev. 2010, 39, 2352-2353.

21. Giridharagopal, R.; Ginger, D.S. Characterizing morphology in bulk heterojunction organic photovoltaic systems. J. Phys. Chem. Lett. 2010, 1, 1160-1169.

22. Chen, W.; Nikiforov, M.P. Morphology characterization in organic and hybrid solar cells. Energy Environ. Sci. 2012, 5, 8045-8074.

23. Rivnay, J.; Mannsfeld, S.C.B. Quantitative determination of organic semiconductor microstructure from the molecular to device scale. Chem. Rev. 2012, 112, 5488-5519.

24. Brinkmann, M. Structure and morphology control in thin films of regioregular poly(3-hexylthiophene). J. Polym. Sci. B Polym. Phys. 2011, 49, 1218-1233.

25. Tsao, H.N.; Mullen, K. Improving polymer transistor performance via morphology control. Chem. Soc. Rev. 2010, 39, 2372-2386.

26. Virkar, A.A.; Mannsfeld, S. Organic semiconductor growth and morphology considerations for organic thin-film transistors. Adv. Mater. 2010, 22, 3857-3875.

27. Knaapila, M.; Monkman, A.P. Methods for controlling structure and photophysical properties in polyfluorene solutions and gels. Adv. Mater. 2013, 25, 1090-1108.

28. Lim, J.A.; Liu, F. Polymer semiconductor crystals. Mater. Today 2010, 13, 14-24.

29. Vacha, M.; Habuchi, S. Conformation and physics of polymer chains: A single-molecule perspective. NPG Asia Mater. 2010, 2, 134-142.

30. Clark, J.; Silva, C. Role of intermolecular coupling in the photophysics of disordered organic semiconductors: Aggregate emission in regioregular polythiophene. Phys. Rev. Lett. 2007, 98, 206406:1-206406:4.

31. Clark, J.; Chang, J.F. Determining exciton bandwidth and film microstructure in polythiophene films using linear absorption spectroscopy. Appl. Phys. Lett. 2009, 94, 163306:1-163306:4.

32. Spano, F.C. The spectral signatures of frenkel polarons in H- and J-Aggregates. Acc. Chem. Res. 2010, 43, 429-439.

33. Oelkrug, D.; Egelhaaf, H.J. Electronic deactivation in single chains, nano-aggregates and ultrathin films of conjugated oligomers. Synth. Metals 1996, 76, 249-253.

34. Spano, F.C. Excitons in conjugated oligomer aggregates, films, and crystals. Annu. Rev. Phys. Chem. 2006, 57, 217-243. 
35. Fidder, H.; Knoester, J. Superradiant emission and optical dephasing in J-Aggregates. Chem. Phys. Lett. 1990, 171, 529-536.

36. Niles, E.T.; Roehling, J.D. J-Aggregate behavior in poly-3-hexylthiophene nanofibers. J. Phys. Chem. Lett. 2012, 3, 259-263.

37. Zhang, X.R.; Richter, L.J. Molecular packing of high-mobility diketo pyrrolo-pyrrole polymer semiconductors with branched alkyl side chains. J. Am. Chem. Soc. 2011, 133, 15073-15084.

38. Lee, H.S.; Lee, J.S. Crystallinity-controlled naphthalene-alt-diketopyrrolopyrrole copolymers for high-performance ambipolar field effect transistors. J. Phys. Chem. C 2012, 116, 26204-26213.

39. Kim, J.W.; Baeg, K.J. Optimal ambipolar charge transport of thienylenevinylene-based polymer semiconductors by changes in conformation for high-performance organic thin film transistors and inverters. Chem. Mater. 2013, 25, 1572-1583.

40. Gao, J.; Kamps, A. Encapsulation of poly(3-hexylthiophene) J-aggregate nanofibers with an amphiphilic block copolymer. Langmuir 2012, 28, 16401-16407.

41. Jimison, L.H.; Toney, M.F. Charge-transport anisotropy due to grain boundaries in directionally crystallized thin films of regioregular poly(3-hexylthiophene). Adv. Mater. 2009, 21, 1568-1572.

42. Lee, M.J.; Gupta, D. Anisotropy of charge transport in a uniaxially aligned and chain-extended, high-mobility, conjugated polymer semiconductor. Adv. Funct. Mater. 2011, 21, 932-940.

43. Lan, Y.K.; Huang, C.I. Charge mobility and transport behavior in the ordered and disordered states of the regioregular poly(3-hexylthiophene). J. Phys. Chem. B 2009, 113, 14555-14564.

44. Kline, R.J.; McGehee, M.D. Dependence of regioregular poly(3-hexylthiophene) film morphology and field-effect mobility on molecular weight. Macromolecules 2005, 38, 3312-3319.

45. Bolsee, J.C.; Oosterbaan, W.D. The importance of bridging points for charge transport in webs of conjugated polymer nanofibers. Adv. Funct. Mater. 2013, 23, 862-869.

46. Street, R.A.; Northrup, J.E. Transport in polycrystalline polymer thin-film transistors. Phys. Rev. $B$ 2005, 71, 165202:1-165202:13.

47. Crossland, E.J.W.; Tremel, K. Anisotropic charge transport in spherulitic poly(3-hexylthiophene) films. Adv. Mater. 2012, 24, 839-844.

48. Zhang, X.R.; Hudson, S.D. In-plane liquid crystalline texture of high-performance thienothiophene copolymer thin films. Adv. Funct. Mater. 2010, 20, 4098-4106.

49. Schuettfort, T.; Watts, B. Microstructure of polycrystalline PBTTT films: Domain mapping and structure formation. ACS Nano 2012, 6, 1849-1864.

50. Liu, J.Y.; Zhang, R. Highly disordered polymer field effect transistors: $N$-alkyl dithieno[3,2-b:2',3'-d]pyrrole-based copolymers with surprisingly high charge carrier mobilities. J. Am. Chem. Soc. 2008, 130, 13167-13176.

51. Lee, J.B.; Kim, K.H. High-performance amorphous donor-acceptor conjugated polymers containing $\mathrm{x}$-shaped anthracene-based monomer and 2,5-bis(2-octyldodecyl)pyrrolo[3,4-c]pyrrole-1,4(2H,5H)dione for organic thin-film transistors. J. Polym. Sci. A Polym. Chem. 2012, 50, 2809-2818.

52. Aiyar, A.R.; Hong, J.I. Tunable crystallinity in regioregular poly(3-hexylthiophene) thin films and its impact on field effect mobility. Adv. Funct. Mater. 2011, 21, 2652-2659.

53. Yu, Z; Fang, J. Self-assembly of well-defined poly(3-hexylthiophene) nanostructures toward the structure-property relationship determination of polymer solar cells. J. Phys. Chem. C 2012, 116, 23858-23863. 
54. Lee, J.S.; Son, S.K. Importance of solubilizing group and backbone planarity in low band gap polymers for high performance ambipolar field-effect transistors. Chem. Mater. 2012, 24, 1316-1323.

55. Kim, D.H.; Ayzner, A.L. Comparison of the photovoltaic characteristics and nanostructure of fullerenes blended with conjugated polymers with siloxane-terminated and branched aliphatic side chains. Chem. Mater. 2013, 25, 431-440.

56. Osaka, I.; Kakara, T. Naphthodithiophene-naphthobisthiadiazole copolymers for solar cells: Alkylation drives the polymer backbone flat and promotes efficiency. J. Am. Chem. Soc. 2013, $135,8834-8837$.

57. Liu, J.G.; Sun, Y. Oriented poly(3-hexylthiophene) nanofibril with the $\pi-\pi$ stacking growth direction by solvent directional evaporation. Langmuir 2011, 27, 4212-4219.

58. Muller, C.; Aghamohammadi, M. One-step macroscopic alignment of conjugated polymer systems by epitaxial crystallization during spin-coating. Adv. Funct. Mater. 2013, 23, 2368-2377.

59. Beniek, L.; Leclerc, N. Large scale alignment and charge transport anisotropy of pBTTT films oriented by high temperature rubbing. Macromolecules 2013, 46, 4014-4023.

60. Lu, G.H.; Li, L.G. Achieving perpendicular alignment of rigid polythiophene backbones to the substrate by using solvent-vapor treatment. Adv. Mater. 2007, 19, 3594-3598.

61. Lu, G.H.; Li, L.G. Morphology and crystalline transition of poly(3-butylthiophene) associated with its polymorphic modifications. Macromolecules 2008, 41, 2062-2070.

62. Aryal, M.; Trivedi, K. Nano-confinement induced chain alignment in ordered P3HT nanostructures defined by nanoimprint lithography. ACS Nano 2009, 3, 3085-3090.

63. Rivnay, J.; Toney, M.F. Unconventional face-on texture and exceptional in-plane order of a high mobility n-type polymer. Adv. Mater. 2010, 22, 4359-4363.

64. DeLongchamp, D.M.; Vogel, B.M. Variations in semiconducting polymer microstructure and hole mobility with spin-coating speed. Chem. Mater.2005, 17, 5610-5612.

65. Yang, H.H.; LeFevre, S.W. Solubility-driven thin film structures of regioregular poly(3-hexyl thiophene) using volatile solvents. Appl. Phys. Lett. 2007, doi:10.1063/1.2734387.

66. Wang, S.; Kiersnowski, A. Microstructure evolution and device performance in solution-processed polymeric field-effect transistors: The key role of the first monolayer. J. Am. Chem. Soc. 2012, 134, 4015-4018.

67. Wang, S.H.; Pisula, W. Nanofiber growth and alignment in solution processed n-type naphthalene-diimide-based polymeric field-effect transistors. J. Mater. Chem. 2012, 22, 24827-24831.

68. Zhao, L.H.; Png, R.Q. Role of borderline solvents to induce pronounced extended-chain lamellar order in $\pi$-stackable polymers. Macromolecules 2011, 44, 9692-9702.

69. Adachi, T.; Brazard, J. Regioregularity and single polythiophene chain conformation. J. Phys. Chem. Lett. 2011, 2, 1400-1404.

70. Mena-Osteritz, E.; Meyer, A. Two-dimensional crystals of poly(3-alkylthiophene)s: Direct visualization of polymer folds in submolecular resolution. Angew. Chem. Int. Ed. 2000, 39, 2680-2684. 
71. Brinkmann, M.; Rannou, P. Molecular weight dependence of chain packing and semicrystalline structure in oriented films of regioregular poly(3-hexylthiophene) revealed by high-resolution transmission electron microscopy. Macromolecules 2009, 42, 1125-1130.

72. Liu, J.; Mikhaylov, I.A. Insight into how molecular structures of thiophene-based conjugated polymers affect crystallization behaviors. Polymer 2011, 52, 2302-2309.

73. Xiao, X.L.; Wang, Z.B. Single crystals of polythiophene with different molecular conformations obtained by tetrahydrofuran vapor annealing and controlling solvent evaporation. J. Phys. Chem. B 2010, 114, 7452-7460.

74. Liu, C.F.; Wang, Q.L. Extended-chain lamellar crystals of monodisperse polyfluorenes. Polymer 2013, 54, 2459-2465.

75. Kim, D.H.; Han, J.T. Single-crystal polythiophene microwires grown by self-assembly. Adv. Mater. 2006, 18, 719-723.

76. Liu, C.F.; Wang, Q.L. Insight into lamellar crystals of monodisperse polyfluorenesFractionated crystallization and the crystal's stability. Polymer 2013, 54, 1251-1258.

77. Rahimi, K.; Botiz, I. Controllable processes for generating large single crystals of poly(3-hexylthiophene). Angew. Chem. Int. Ed. 2012, 51, 11131-11135.

78. Kim, J.H.; Lee, D.H. Novel polymer nanowire crystals of diketopyrrolopyrrole-based copolymer with excellent charge transport properties. Adv. Mater. 2013, 25, 4102-4106.

79. Wang, H.Y.; Liu, J.G. Fibrillar morphology of derivatives of poly(3-alkylthiophene)s by solvent vapor annealing: Effects of conformational transition and conjugate length. J. Phys. Chem. B 2013, 117, 5996-6006.

80. Hultell, M.; Stafstrom, S. Impact of ring torsion on the intrachain mobility in conjugated polymers. Phys. Rev. B 2007, 75, 104304:1-104304:7.

81. Cho, H.H.; Kang, T.E. Effect of incorporated nitrogens on the planarity and photovoltaic performance of donor-acceptor copolymers. Macromolecules 2012, 45, 6415-6423.

82. Ong, B.S.; Wu, Y. Thiophene polymer semiconductors for organic thin-film transistors. Chem. Eur. J. 2008, 14, 4766-4778.

83. Kim, J.; Lim, B. Highly soluble poly(thienylenevinylene) derivatives with charge-carrier mobility exceeding $1 \mathrm{~cm}^{2} \cdot \mathrm{V}^{-1} \cdot \mathrm{s}^{-1}$. Chem. Mater. 2011, 23, 4663-4665.

84. Lee, W.H.; Kong, H. Field-effect transistors based on ppv derivatives as a semiconducting layer. J. Polym. Sci. A Polym. Chem. 2009, 47, 111-120.

85. Stevens, D.M.; Qin, Y. Enhancement of the morphology and open circuit voltage in bilayer polymer/fullerene solar cells. J. Phys. Chem. C 2009, 113, 11408-11415.

86. Lei, T.; Cao, Y. Systematic investigation of isoindigo-based polymeric field-effect transistors: Design strategy and impact of polymer symmetry and backbone curvature. Chem. Mater. 2012, 24, 1762-1770.

87. Ong, B.S.; Wu, Y.L. Structurally ordered polythiophene nanoparticles for high-performance organic thin-film transistors. Adv. Mater. 2005, 17, 1141-1144.

88. McCulloch, I.; Heeney, M. Liquid-crystalline semiconducting polymers with high charge-carrier mobility. Nat. Mater. 2006, 5, 328-333.

89. Li, J.; Qin, F. High-performance thin-film transistors from solution-processed dithienothiophene polymer semiconductor nanoparticles. Chem. Mater. 2008, 20, 2057-2059. 
90. Pan, H.L.; Li, Y.N. Low-temperature, solution-processed, high-mobility polymer semiconductors for thin-film transistors. J. Am. Chem. Soc. 2007, 129, 4112-4113.

91. Osaka, I.; Abe, T. High-mobility semiconducting naphthodithiophene copolymers. J. Am. Chem. Soc. 2010, 132, 5000-5001.

92. Osaka, I.; Zhang, R. High-lamellar ordering and amorphous-like $\pi$-network in short-chain thiazolothiazole-thiophene copolymers lead to high mobilities. J. Am. Chem. Soc. 2009, 131, 2521-2529.

93. Zhang, M.X.; Zhao, G.J. Heteroatomic effects on charge-transfer mobility of dianthra[2,3-b:2',3'-f] thieno[3,2-b]thiophene (DATT) and its derivatives. J. Phys. Chem. C 2012, 116, 19197-19202.

94. Kang, I.; An, T.K. Effect of selenophene in a DPP copolymer incorporating a vinyl group for high-performance organic field-effect transistors. Adv. Mater. 2013, 25, 524-528.

95. Lin, Y.Z.; Fan, H.J. Thiazole-based organic semiconductors for organic electronics. Adv. Mater. 2012, 24, 3087-3106.

96. Osaka, I.; Saito, M. Drastic change of molecular orientation in a thiazolothiazole copolymer by molecular-weight control and blending with $\mathrm{PC}_{61} \mathrm{BM}$ leads to high efficiencies in solar cells. Adv. Mater. 2012, 24, 425-430.

97. Chen, Z.Y.; Lemke, H. High mobility ambipolar charge transport in polyselenophene conjugated polymers. Adv. Mater. 2010, 22, 2371-2375.

98. Zhang, W.M.; Smith, J. Indacenodithiophene semiconducting polymers for high-performance, air-stable transistors. J. Am. Chem. Soc. 2010, 132, 11437-11439.

99. Guo, X.G.; Kim, F.S. Naphthalene diimide-based polymer semiconductors: Synthesis, structure-property correlations, and n-channel and ambipolar field-effect transistors. Chem. Mater. 2012, 24, 1434-1442.

100. Yan, H.; Chen, Z.H. A high-mobility electron-transporting polymer for printed transistors. Nature 2009, 457, 679-686.

101. Zhao, X.G.; Zhan, X.W. Electron transporting semiconducting polymers in organic electronics. Chem. Soc. Rev. 2011, 40, 3728-3743.

102. Li, Y.N.; Sonar, P. High mobility diketopyrrolopyrrole (DPP)-based organic semiconductor materials for organic thin film transistors and photovoltaics. Energy Environ. Sci. 2013, 6, 1684-1710.

103. Beaujuge, P.M.; Amb, C.M. Spectral engineering in $\pi$-conjugated polymers with intramolecular donor-acceptor interactions. Acc. Chem. Res. 2010, 43, 1396-1407.

104. Ha, J.S.; Kim, K.H. 2,5-Bis(2-octyldodecyl)pyrrolo[3,4-c]pyrrole-1,4-(2H,5H)-dione-based donor-acceptor alternating copolymer bearing 5,5'-di(thiophen-2-yl)-2,2'-biselenophene exhibiting $1.5 \mathrm{~cm}^{2} \cdot \mathrm{V}^{-1} \cdot \mathrm{s}^{-1}$ hole mobility in thin-film transistors. J. Am. Chem. Soc. 2011, 133, 10364-10367.

105. Chen, Z.Y.; Lee, M.J. High-performance ambipolar diketopyrrolopyrrole-thieno[3,2-b]thiophene copolymer field-effect transistors with balanced hole and electron mobilities. Adv. Mater. 2012, 24, 647-652.

106. Chen, H.J.; Guo, Y.L. Highly $\pi$-extended copolymers with diketopyrrolopyrrole moieties for high-performance field-effect transistors. Adv. Mater. 2012, 24, 4618-4622.

107. Lei, T.; Dou, J.H. Electron-deficient poly ( $p$-phenylene vinylene) provides electron mobility over $1 \mathrm{~cm}^{2} \cdot \mathrm{V}^{-1} \cdot \mathrm{s}^{-1}$ under ambient conditions. J. Am. Chem. Soc. 2013, 135, 12168-12171. 
108. Sauvé, G.; Javier, A.E. Well-defined, high molecular weight poly(3-alkylthiophene)s in thin-film transistors: side chain invariance in field-effect mobility. J. Mater. Chem. 2010, 20, 3195-3201.

109. Hu, Z.J.; Liu, J.H. Influence of backbone rigidness on single chain conformation of thiophene-based conjugated polymers. J. Phys. Chem. B 2013, 117, 4461-4467.

110. Beaujuge, P.M.; Pisula, W. Tailoring structure-property relationships in dithienosilole-benzothiadiazole donor-acceptor copolymers. J. Am. Chem. Soc. 2009, 131, 7514-7515.

111. Deng, Y.F.; Chen, Y.G. Donor-acceptor conjugated polymers with dithienocarbazoles as donor units: Effect of structure on semiconducting properties. Macromolecules 2012, 45, 8621-8627.

112. Osaka, I.; Abe, T. Impact of isomeric structures on transistor performances in naphthodithiophene semiconducting polymers. J. Am. Chem. Soc. 2011, 133, 6852-6860.

113. Brinkmann, M.; Gonthier, E. Segregated vs. mixed interchain stacking in highly oriented films of naphthalene diimide bithiophene copolymers. ACS Nano 2012, 6, 10319-10326.

114. Donley, C.L.; Zaumseil, J. Effects of packing structure on the optoelectronic and charge transport properties in poly(9,9-di-n-octylfluorene-alt-benzothiadiazole). J. Am. Chem. Soc. 2005, 127, 12890-12899.

115. Park, J.W.; Lee, D.H. Conformationally twisted semiconducting polythiophene derivatives with alkylthiophene side chain: High solubility and air stability. Macromolecules 2010, 43, 2118-2123.

116. Schroeder, B.C.; Nielsen, C.B. Benzotrithiophene co-polymers with high charge carrier mobilities in field-effect transistors. Chem. Mater. 2011, 23, 4025-4031.

117. Ren, X.K.; Wu, Y.C. Crystal structure and molecular packing behavior of poly(2,3-diphenyl-1,4-phenylenevinylene) derivatives containing alkyl side-chains. Macromolecules 2013, 46, 155-163.

118. Bolognesi, A.; Botta, C. Oriented thin films from soluble polythiophenes. Polym. Adv. Technol. 2003, 14, 537-543.

119. Nagamatsu, S.; Misaki, M. Side-chain effects on friction-transferred polymer orientation. Polym. J. 2007, 39, 1300-1305.

120. Higashi, T.; Yamasaki, N. Anisotropic properties of aligned $\pi$-conjugated polymer films fabricated by capillary action and their post-annealing effects. Appl. Phys. Express 2011, doi:10.1143/APEX.4.091602.

121. He, M.Q.; Li, J.F. Alkylsubstituted thienothiophene semiconducting materials: Structure-property relationships. J. Am. Chem. Soc. 2009, 131, 11930-11938.

122. Rieger, R.; Beckmann, D. Backbone curvature in polythiophenes. Chem. Mater. 2010, 22, 5314-5318.

123. Yazawa, K.; Inoue, Y. Molecular dynamics of regioregular poly(3-hexylthiophene) investigated by $\mathrm{nmr}$ relaxation and an interpretation of temperature dependent optical absorption. J. Phys. Chem. B 2010, 114, 1241-1248.

124. Oosterbaan, W.D.; Bolsee, J.C. Alkyl-chain-length-independent hole mobility via morphological control with poly(3-alkylthiophene) nanofibers. Adv. Funct. Mater. 2010, 20, 792-802.

125. Pankaj, S.; Beiner, M. Confined dynamics and crystallization in self-assembled alkyl nanodomains. J. Phys. Chem. B 2010, 114, 15459-15465.

126. Malik, S.; Nandi, A.K. Crystallization mechanism of regioregular poly(3-alkyl thiophene)s. J. Polym. Sci. B Polym. Phys. 2002, 40, 2073-2085. 
127. Li, Z.; Tsang, S.W. Alternating copolymers of cyclopenta[2,1-b;3,4-b'] dithiophene and thieno[3,4-c]pyrrole-4,6-dione for high-performance polymer solar cells. Adv. Funct. Mater. 2011, 21, 3331-3336.

128. Yamamoto, T.; Ikai, T. Synthesis and characterization of thieno[3,4- $b]$ thiophene-based copolymers bearing 4-substituted phenyl ester pendants: Facile fine-tuning of HOMO energy levels. Macromolecules 2011, 44, 6659-6662.

129. Kline, R.J.; DeLongchamp, D.M. Critical role of side-chain attachment density on the order and device performance of polythiophenes. Macromolecules 2007, 40, 7960-7965.

130. Wang, C.C.; Jimison, L.H. Microstructural origin of high mobility in high-performance poly(thieno-thiophene) thin-film transistors. Adv. Mater. 2010, 22, 697-701.

131. Keg, P.; Lohani, A. Direct observation of alkyl chain interdigitation in conjugated polyquarterthiophene self-organized on graphite surfaces. Macromole. Rapid Commun. 2008, 29, 1197-1202.

132. Meager, L.; Ashraf, R.S. Photocurrent Enhancement from diketopyrrolopyrrole polymer solar cells through alkyl-chain branching point manipulation. J. Am. Chem. Soc. 2013, 135, $11537-11540$.

133. Kim, D.H.; Lee, J. Molecular weight-induced structural transition of liquid-crystalline polymer semiconductor for high-stability organic transistor. Adv. Funct. Mater. 2011, 21, 4442-4447.

134. Wu, S. Chain structure and entanglement. J. Polym. Sci. B Polym. Phys. 1989, 27, 723-741.

135. Wen, Y.H.; Lin, P.C. Dynamic structure factor for large aggregate clusters with internal motions: A self-consistent light-scattering study on conjugated polymer solutions. J. Phys. Chem. B 2011, $115,14369-14380$.

136. Huang, Y.; Cheng, H. Temperature induced structure evolution of regioregular poly(3-hexylthiophene) in dilute solution and its influence on thin film morphology. Macromolecules 2010, 43, 10031-10037.

137. Huang, Y.; Cheng, H. Unimer-aggregate equilibrium to large scale association of regioregular poly(3-hexylthiophene) in THF solution. Macromolecules 2011, 44, 5020-5026.

138. Rughooputh, S.D.D.V.; Hotta, S. Chromism of soluble polythienylenes. J. Polym. Sci. B Polym. Phys. 1987, 25, 1071-1078.

139. Vogelsang, J.; Brazard, J. Watching the annealing process one polymer chain at a time. Angew. Chem. Int. Ed. 2011, 50, 2257-2261.

140. Steyrleuthner, R.; Schubert, M. Aggregation in a high-mobility n-type low-bandgap copolymer with implications on semicrystalline morphology. J. Am. Chem. Soc. 2012, 134, 18303-18317.

141. Kohler, A.; Hoffmann, S.T. An order-disorder transition in the conjugated polymer MEH-PPV. J. Am. Chem. Soc. 2012, 134, 11594-11601.

142. Malik, S.; Nandi, A.K. Influence of alkyl chain length on the gelation mechanism of thermoreversible gels of regioregular poly(3-alkyl thiophenes) in xylene. J. Appl. Polym. Sci. 2007, 103, 2528-2537.

143. Xu, Y.Z.; Liu, J.G. Formation of parallel aligned nano-fibrils of poly(3,3'"'-didodecylquaterthiophene) induced by the unimer coils in solution. RSC $A d v$. 2013, 3, 12069-12074. 
144. Samitsu, S.; Shimomura, T. Effective production of poly(3-alkylthiophene) nanofibers by means of whisker method using anisole solvent: structural, optical, and electrical properties. Macromolecules 2008, 41, 8000-8010.

145. Xue, L.J.; Gao, X. The formation of different structures of poly(3-hexylthiophene) film on a patterned substrate by dip-coating from aged solution. Nanotechnology 2010, doi:10.1088/0957-4484/21/14/145303.

146. Zhao, K.; Xue, L. A new method to improve poly(3-hexyl thiophene) (P3HT) crystalline behavior: Decreasing chains entanglement to promote order-disorder transformation in solution. Langmuir 2010, 26, 471-477.

147. Zhao, K.; Khan, H.U. Entanglement of conjugated polymer chains influences molecular self-assembly and carrier transport. Adv. Funct. Mater. 2013, doi:10.1002/adfm.201301007.

148. Wang, P.S.; Lu, H.H. Gel formation via physical cross-linking in the soluble conjugated polymer, poly[2-methoxy-5-(2-ethylhexyloxy)-1,4-phenylenevinylene], in solution by addition of alkalies. Macromolecules 2008, 41, 6500-6504.

149. Liu, J.; Shao, S. The mechanisms for introduction of n-dodecylthiol to modify the P3HT/PCBM morphology. Org. Electron. 2010, 11, 775-783.

150. Wang, H.Y.; Liu, J.G. Nano-fibrils formation of pBTTT via adding alkylthiol into solutions: Control of morphology and crystalline structure. Polymer 2013, 54, 948-957.

151. Traiphol, R.; Charoenthai, N. Chain organization and photophysics of conjugated polymer in poor solvents: Aggregates, agglomerates and collapsed coils. Polymer 2007, 48, 813-826.

152. Oh, J.Y.; Shin, M. Self-seeded growth of poly(3-hexylthiophene) (P3HT) nanofibrils by a cycle of cooling and heating in solutions. Macromolecules 2012, 45, 7504-7513.

153. Li, L.G.; Lu, G.H. Improving performance of polymer photovoltaic devices using an annealing-free approach via construction of ordered aggregates in solution. J. Mater. Chem. 2008, 18, 1984-1990.

154. Park, Y.D.; Lee, H.S. Solubility-induced ordered polythiophene precursors for high-performance organic thin-film transistors. Adv. Funct. Mater.2009, 19, 1200-1206.

155. Yan, H.; Yan, Y. Self-assembling branched and hyperbranched nanostructures of poly(3-hexylthiophene) by a solution process. J. Phys. Chem. C 2011, 115, 3257-3262.

156. Xu, W.; Li, L. Solvent-induced crystallization of poly(3-dodecylthiophene): Morphology and kinetics. J. Phys. Chem. B 2011, 115, 6412-6420.

157. Berson, S.; de Bettignies, R. Poly(3-hexylthiophene) fibers for photovoltaic applications. Adv. Funct. Mater. 2007, 17, 1377-1384.

158. He, M.; Ge, J. Fabricating polythiophene into highly aligned microwire film by fast evaporation of its whisker solution. Polymer 2010, 51, 2236-2243.

159. Crossland, E.J.W.; Rahimi, K. Systematic control of nucleation density in poly(3-hexylthiophene) thin films. Adv. Funct. Mater.2011, 21, 518-524.

160. Lu, G.H.; Tang, H.W. Enhanced charge transportation in semiconducting polymer/insulating polymer composites: The role of an interpenetrating bulk interface. Adv. Funct. Mater.2010, 20, 1714-1720.

161. Benetti, E.M.; Causin, V. Conjugated polymers in cages: Templating poly(3-hexylthiophene) nanocrystals by inert gel matrices. Adv. Mater. 2012, 24, 5636-5641. 
162. Kim, F.S.; Jenekhe, S.A. Charge transport in poly(3-butylthiophene) nanowires and their nanocomposites with an insulating polymer. Macromolecules 2012, 45, 7514-7519.

163. Qiu, L.Z.; Lee, W.H. Organic thin-film transistors based on polythiophene nanowires embedded in insulating polymer. Adv. Mater. 2009, 21, 1349-1353.

164. Qiu, L.Z.; Wang, X. Organic thin-film transistors based on blends of poly(3-hexylthiophene) and polystyrene with a solubility-induced low percolation threshold. Chem. Mater. 2009, 21, 4380-4386.

165. Kim, D.H.; Park, Y.D. Solvent vapor-induced nanowire formation in poly(3-hexylthiophene) thin films. Macromol. Rapid Commun. 2005, 26, 834-839.

166. Chang, J.F.; Sun, B.Q. Enhanced mobility of poly(3-hexylthiophene) transistors by spin-coating from high-boiling-point solvents. Chem. Mater. 2004, 16, 4772-4776.

167. Wang, S.H.; Kappl, M. Organic field-effect transistors based on highly ordered single polymer fibers. Adv. Mater. 2012, 24, 417-420.

168. Dong, H.L.; Jiang, S.D. Nanowire crystals of a rigid rod conjugated polymer. J. Am. Chem. Soc. 2009, 131, 17315-17320.

169. Liu, C.F.; Wang, Q.L. Morphology and structure of the $\beta$ phase crystals of monodisperse polyfluorenes. Macromolecules 2013, 46, 3025-3030.

170. Liu, Y.; Dong, H.L. High performance nanocrystals of a donor-acceptor conjugated polymer. Chem. Mater. 2013, 25, 2649-2655.

171. Jo, J.; Kim, S.S. Time-dependent morphology evolution by annealing processes on polymer: Fullerene blend solar cells. Adv. Funct. Mater. 2009, 19, 866-874.

172. Vogelsang, J.; Lupton, J.M. Solvent vapor annealing of single conjugated polymer chains: Building organic optoelectronic materials from the bottom up. J. Phys. Chem. Lett. 2012, 3, $1503-1513$.

173. Liu, Y.; Shi, Q.Q. Solvent-vapor induced self-assembly of a conjugated polymer: A correlation between solvent nature and transistor performance. Org. Electron. 2012, 13, 2372-2378.

174. Liu, J.G.; Chen, L. Constructing the nanointerpenetrating structure of PCDTBT:PC70BM bulk heterojunction solar cells induced by aggregation of $\mathrm{PC}_{70} \mathrm{BM}$ via mixed-solvent vapor annealing. J. Mater. Chem. A 2013, 1, 6216-6225.

(C) 2013 by the authors; licensee MDPI, Basel, Switzerland. This article is an open access article distributed under the terms and conditions of the Creative Commons Attribution license (http://creativecommons.org/licenses/by/3.0/). 